\title{
Development and Testing of a High Resolution PET Detector for Prostate Imaging
}

Evan P. Delfino

West Virginia University

Follow this and additional works at: https://researchrepository.wvu.edu/etd

\section{Recommended Citation}

Delfino, Evan P., "Development and Testing of a High Resolution PET Detector for Prostate Imaging" (2012). Graduate Theses, Dissertations, and Problem Reports. 3509.

https://researchrepository.wvu.edu/etd/3509

This Thesis is protected by copyright and/or related rights. It has been brought to you by the The Research Repository @ WVU with permission from the rights-holder(s). You are free to use this Thesis in any way that is permitted by the copyright and related rights legislation that applies to your use. For other uses you must obtain permission from the rights-holder(s) directly, unless additional rights are indicated by a Creative Commons license in the record and/ or on the work itself. This Thesis has been accepted for inclusion in WVU Graduate Theses, Dissertations, and Problem Reports collection by an authorized administrator of The Research Repository @ WVU. For more information, please contact researchrepository@mail.wvu.edu. 


\title{
Development and Testing of a High Resolution PET Detector for Prostate Imaging
}

\author{
Evan P. Delfino
}

Thesis submitted to the Eberly College of Arts and Sciences

at West Virginia University in partial fulfillment

of requirements for the degree of

\author{
Masters of Science \\ in \\ Physics
}

\begin{abstract}
Raymond R. Raylman, Ph.D, Chair Stanislaw Majewski, Ph.D, Advisor

Alan D. Bristow, Ph.D
\end{abstract}

Department of Physics

Morgantown, West Virginia
2012

Keywords: Prostate Cancer; Positron Emission Tomography (PET); Silicon Photomultiplier (SiPM); Depth of Interaction (DOI)

Copyright 2012 Evan P. Delfino 


\title{
ABSTRACT \\ Development and Testing of a High Resolution PET Detector for Prostate Imaging
}

\author{
Evan P. Delfino
}

According to the American Cancer Society one in six men will be diagnosed with prostate cancer in their lifetime. Current methods for screening of prostate cancer including various PSA blood tests, as well as the digital rectal exam, are unreliability while current imaging modalities clinically employed (US, CT, MRI) are unable to localize intraprostatic cancer(s). Consequently, diagnosis via core needle biopsy is problematic and a game of chance at best. Therefore, in response to new radiopharmaceuticals applicable to both internal and external prostate cancer visualization and localization, novel prostate specific nuclear medical imagers are being developed.

The first prototype of a compact prostate specific PET detector utilizing silicon photomultiplier (SiPM) technology has been developed and tested at West Virginia University. The compact detector is proposed as an endorectal probe placed proximally to the rectal wall/prostate interface and operating in coincidence with one or more externally mounted large area gamma detectors or in tandem with a clinical whole body PET scanner. To ensure high reconstruction resolution, the scintillation array of the compact detector will be coupled to SiPMs on both axial ends in a dual ended readout approach. Such an approach allows for the extraction of continuous depth of interaction (DOI) information thus minimizing the effects of parallax error and providing nearly isotropic and uniform spatial resolution throughout the entire detector field of view (FOV).

Two compact DOI based prototype detectors were developed and tested. While both utilize pixelated LYSO scintillation crystal arrays, the first has a crystal pitch of $1.0 \mathrm{~mm}$ and is coupled to SensL SiPMs, while the second has a crystal pitch of $0.7 \mathrm{~mm}$ and is coupled to Hamamatsu SiPMs. Initial proof of concept studies were preformed using the SensL based detector while more extensive and systematic studies were preformed using the Hamamatsu based detector. Ultimately, when averaged over all crystals and all depths the Hamamatsu based detector achieved a depth of interaction resolution of $0.78 \pm 0.09 \mathrm{~mm}$ FWHM and an energy resolution of $13.2 \pm 0.7 \%$ FWHM. Validation studies with regards to the efficacy of incorporating DOI information extracted from a small compact DOI based PET detector module into image reconstruction algorithms were also preformed. 


\section{Table of Contents}

Chapter 1 - Radioactive Decay and Photon Interactions in Matter ............................................. 1

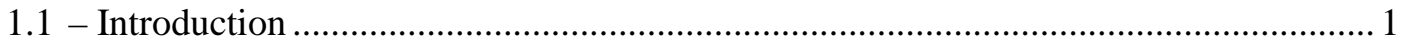

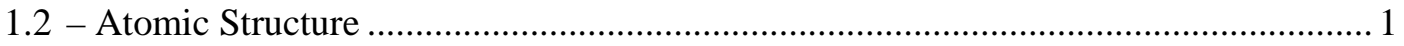

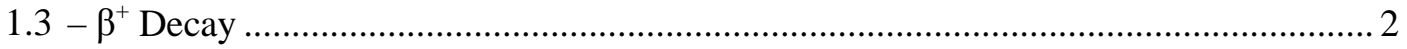

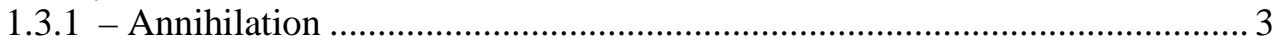

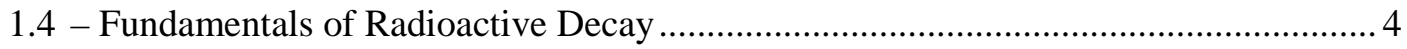

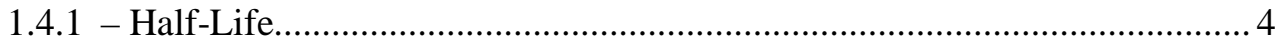

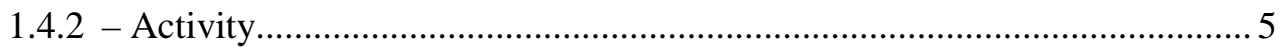

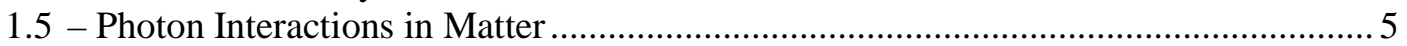

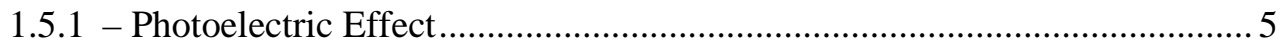

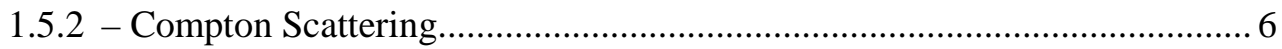

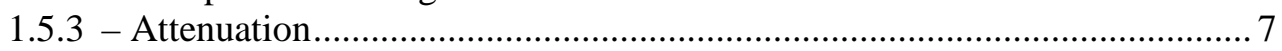

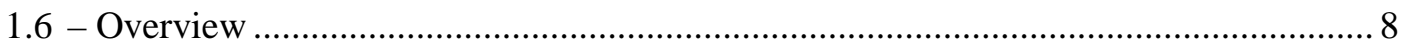

Chapter 2 - Scintillation Detector Principals and Properties ....................................................... 10

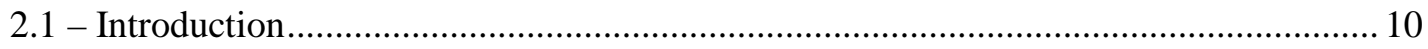

2.2 - Organic Scintillator Band Structure and the

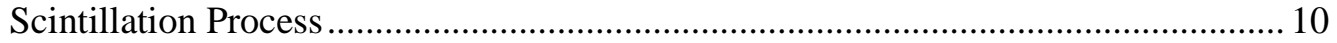

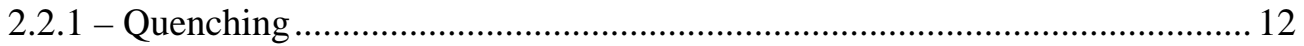

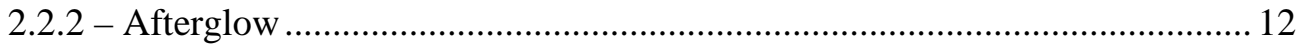

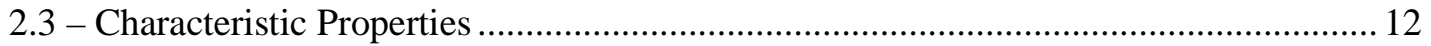

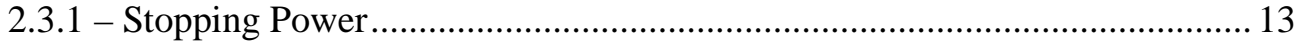

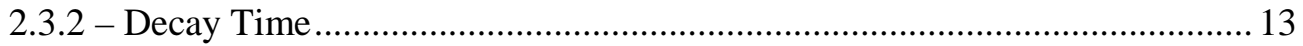

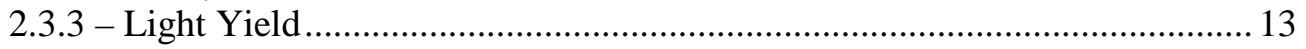

2.3.4 - Intrinsic Energy Resolution ................................................................... 14

2.3.5 - Transparency.......................................................................................... 15

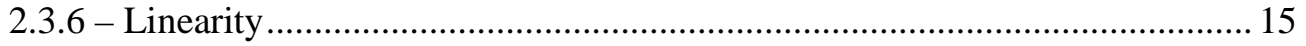

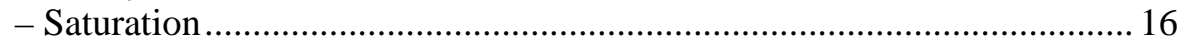

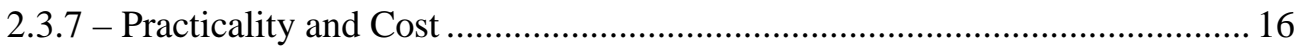

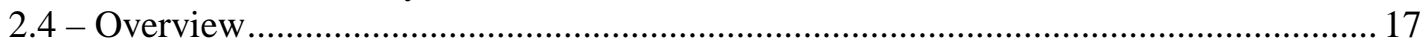

Chapter 3 - Silicon Photomultiplier Design, Structure, and Operation ......................................... 19

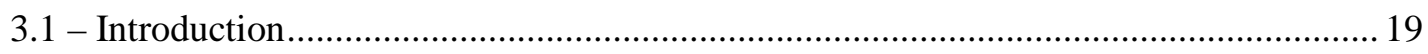

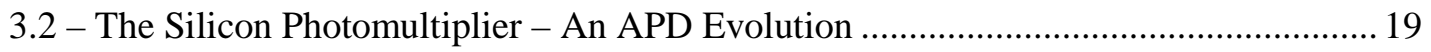

3.2.1 - n-type and p-type Semiconductors........................................................... 20

3.2.2 - p-n Junction................................................................................................. 21

3.2.3 - APD Structure and Operation ..................................................................... 24

3.2.4 - Modes of Operation ..................................................................................... 25

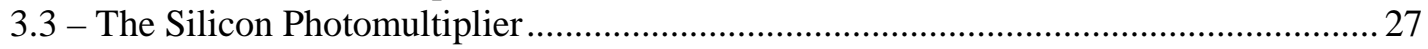

3.3.1 - Photon Detection Efficiency ……............................................................. 27

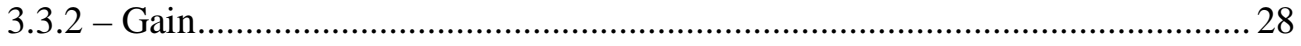

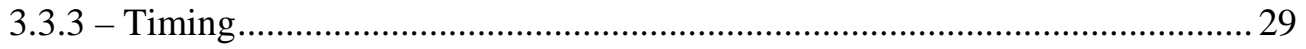

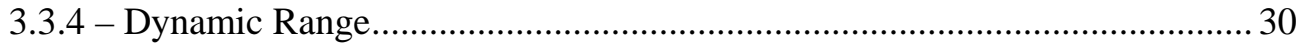

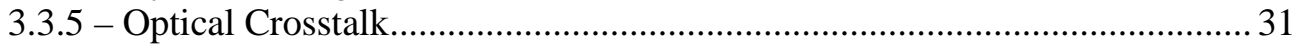

3.3.6 - Low Magnetic Field Sensitivity................................................................... 31

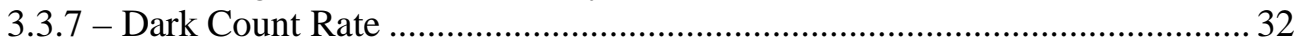

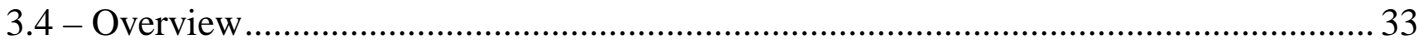




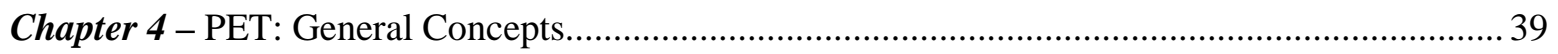

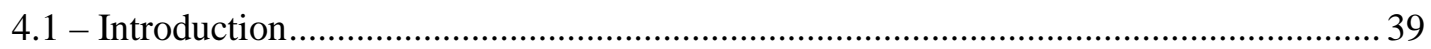

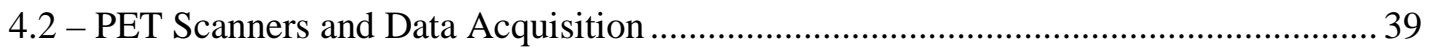

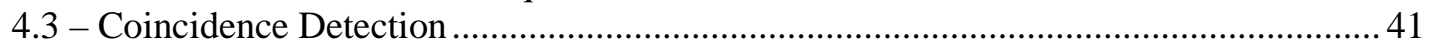

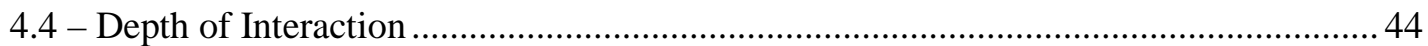

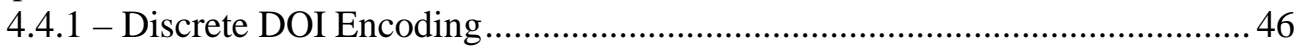

4.4.1.1 - Phoswich Detectors ............................................................................. 46

4.4.1.2 - Light Sharing Detectors............................................................... 47

4.4.1.3 - Relative Offset Detectors ................................................................. 49

4.4.1.4 - Direct DOI Encoding Detector.......................................................... 50

4.4.2 - Continuous DOI Encoding.................................................................. 51

4.4.2.1 - Dual Ended Readout Detectors ....................................................... 51

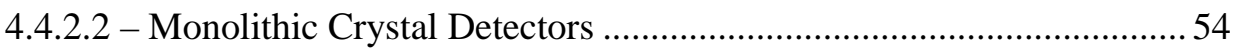

4.4.2.3 - Phosphor Coated Scintillator Detector ............................................. 55

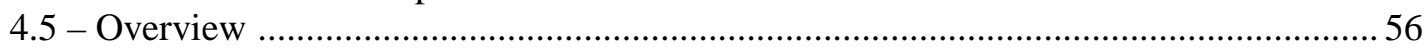

Chapter 5 - Prostate Cancer and Nuclear Medicine.................................................................... 61

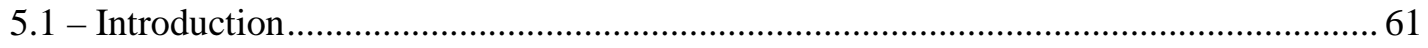

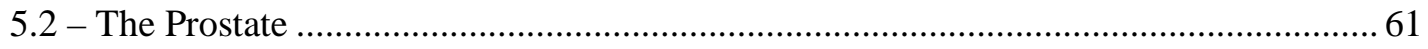

5.2.1 - Anatomy and Histology of the Prostate ............................................................ 61

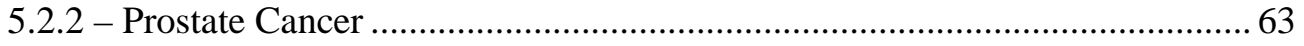

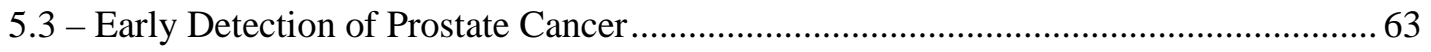

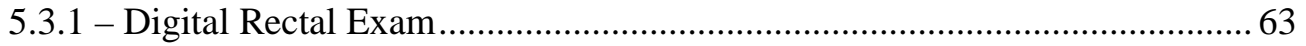

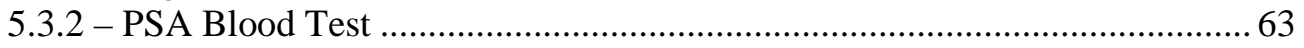

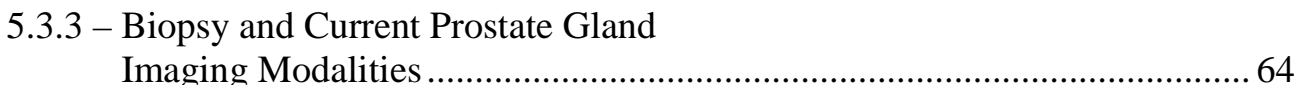

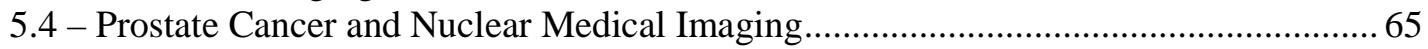

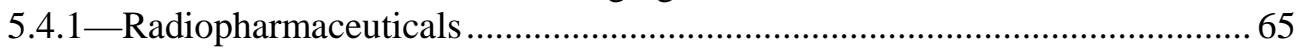

5.4.2 - Prostate Specific Nuclear Medical

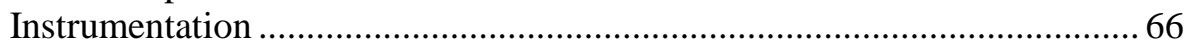

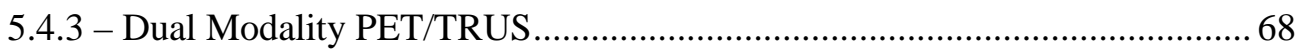

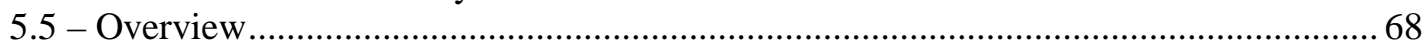

Chapter 6 - Performance Evaluation of a Prostate Specific PET Detector..................................... 74

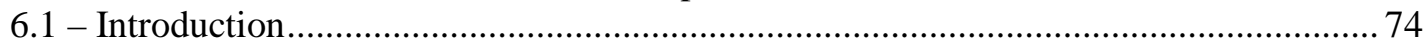

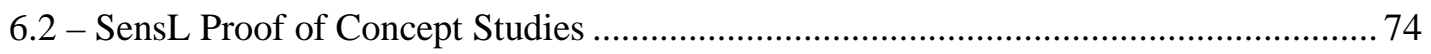

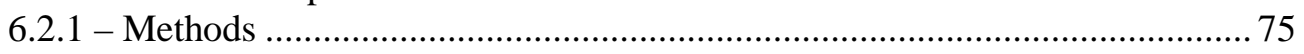

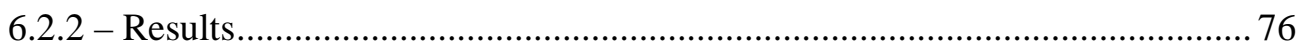

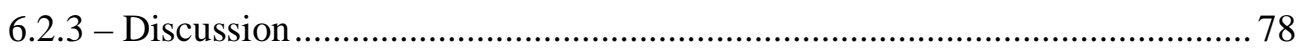

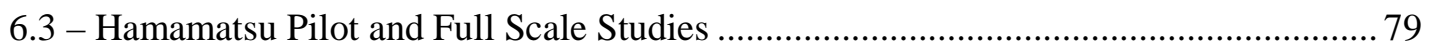

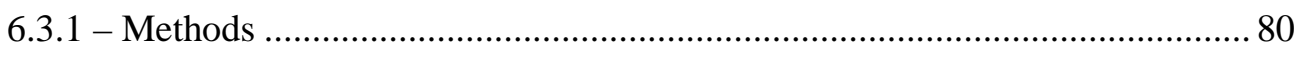

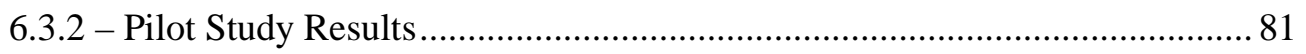

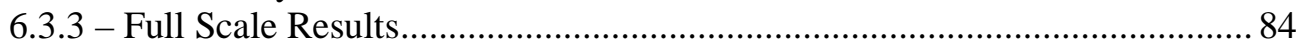

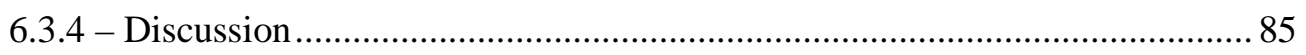

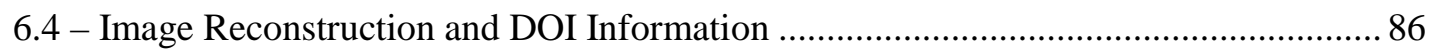

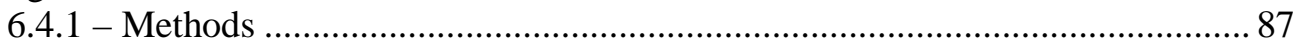

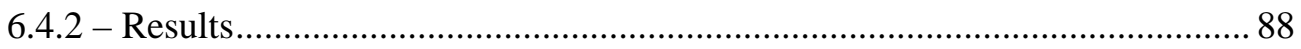

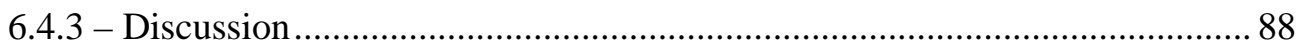

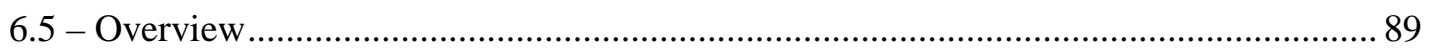




\section{1 \\ Radioactive Decay and Photon Interactions in Matter}

\subsection{Introduction}

Positron emission tomography (PET) is a nuclear medical imaging modality which uses a specialized camera to produce functional images of metabolic activity, blood flow, and chemical processes in the body. The foundation for (PET) begins at the subatomic level. Therefore, this chapter will first explore the atomic structure of the atom. Next, an analysis of how certain unstable atoms, known as radionuclides, decay (specifically $\beta^{+}$decay) will be outlined, followed by an examination of the governing laws by which the decay process abides. Finally, the mechanisms by which the subsequently emitted radiation from the decay process interacts with matter will be presented.

Ultimately, the goal of this chapter is to begin to develop a framework for how the natural phenomenon of radioactive decay and radiation interactions in matter are exploited and utilized in PET.

\subsection{Atomic Structure}

All matter is composed of atoms. The planetary model of the atom proposed by Bohr in 1913 consists of a nucleus comprised of two different types of nucleons; protons and neutrons, as well as a cloud of electrons orbiting the nucleus at discrete radii. Protons and neutrons are of similar mass ( 1.67x10-27 kg) but differ in that a proton is positively charged $(\sim 1.6 \times 10-19 \mathrm{C})$ while a neutron is uncharged. The total number of nucleons is known as the mass number and denoted by the letter $\mathrm{A}$. The electron on the other hand carries significantly less mass $(\sim 9.11 \times 10-31 \mathrm{~kg})$ and is negatively charged $(\sim-1.6 \times 10-19 \mathrm{C})$. In a non-ionized atom the number of protons, knows as the atomic number and denoted by the letter $\mathrm{Z}$, equals the number of electrons. The discrete radii in which the electrons orbit the nucleus are known as energy shells and labeled K-Q from inner most to outermost shell. Each shell is composed of subshells referred to as atomic orbitals labeled as s, p, d, f, and g. For example the K shell has an s orbital, the $\mathrm{L}$ shell has both s and p orbitals, the $\mathrm{M}$ shell has s, p, and d orbitals, etc. (Saha, 1). Each atomic orbital can only accommodate a specific number of electrons; 2, 6, 10, 14, and 18 respectively. Therefore each shell is associated with a particular range of electron energies and must be filled before electrons may occupy an outer shell.

An atom with a specific atomic number is known as a nuclide and determines to which element the atom belongs. Nuclides are defined by the following notation:

$$
{ }_{\mathrm{Z}}^{\mathrm{A}} \mathrm{X}
$$

with $\mathrm{X}$ being the one or two letter symbol of the element with mass number $\mathrm{A}$ and atomic number $\mathrm{Z}$.

The nucleus of each nuclide is held together by two opposing forces; the strong force which is an attractive force between nucleons and the electromagnetical repulsive force between constituent protons in the nucleus. In a stable nucleus these forces are at equilibrium, however when there is an excess of either protons or neutrons the nucleus become unstable and is prone to radioactive decay. Nuclides with equal number of protons but different number of neutrons are termed isotopes, those with equal number 
of neutrons and different number of protons are termed isotones, those with equal mass number but different atomic number are termed isobars, and those with equal mass number but in different states of excitation are termed isomers. These unstable nuclides are called radionuclides and undergo decay by emission of particulate radiation such as $\alpha$ particles, $\beta^{-}$particles, $\beta^{+}$particles (positrons), or through electron capture or isometric transition. The foundation for positron emission tomography (PET) imaging is based upon exploiting decay by positron emission and detecting the collinear (optimally) or nearly collinear $511 \mathrm{keV}$ photons subsequently produced. Therefore only $\beta^{+}$decay will be expanded on further.

\section{$\underline{1.3 \beta^{+} \text {Decay }}$}

A proton rich radionuclide may decay by either positron emission $\left(\beta^{+}\right.$decay) or electron capture. In the case of $\beta^{+}$decay, the proton rich radionuclide converts a bound proton into a bound neutron and in the process emits a positron and a neutrino (eq. 1.2). A positron is the antimatter conjugate of an electron with the same mass but opposite charge.

$$
\mathrm{p} \rightarrow \mathrm{n}+\beta^{+}+\mathrm{v}
$$

The general equation for $\beta^{+}$decay is:

$$
\underset{\mathrm{Z}}{\mathrm{A}} \mathrm{X} \rightarrow{ }_{\mathrm{E}-1}^{\mathrm{A}} \mathrm{Y}+{ }_{1}^{0} \beta^{+}+\mathrm{V}+\text { energI }
$$

However in order for this decay to occur the nuclear mass-energy relationship must first be satisfied (Williams, 71-73). Note that if this condition is not satisfied the radionuclide may still decay by electron capture. In terms of nuclear masses $\mathrm{M}(\mathrm{Z}, \mathrm{A})$ and free energy $\mathrm{Q}$, the mass-energy relationship for $\beta^{+}$ decay appears as:

$$
Q_{B^{+}}=\left(M(Z, A)-M(Z-1, A)-m_{e}\right) c^{2}
$$

A more conventional form of equation 1.4 involves reformulating the condition in terms of atomic masses $\therefore(\mathrm{Z}, \mathrm{A})$ rather than nuclear masses. Substituting

$$
M(Z, A)=M(Z, A)+Z m_{E} \text { and } M(Z-1, A)=M(Z-1, A)+(Z-1) m_{=} \quad 1.5,1.6
$$

equation 1.3 becomes

$$
Q_{B^{*}}=\left(M(Z, A)-M(Z-1, A)-2 m_{e}\right) c^{2}
$$

From equation 1.7 it becomes apparent that the atomic masses of the parent and daughter nuclide must differ by more than twice the electron mass. This is illustrated in the energy level diagram depicted in Fig. 1.1 below.

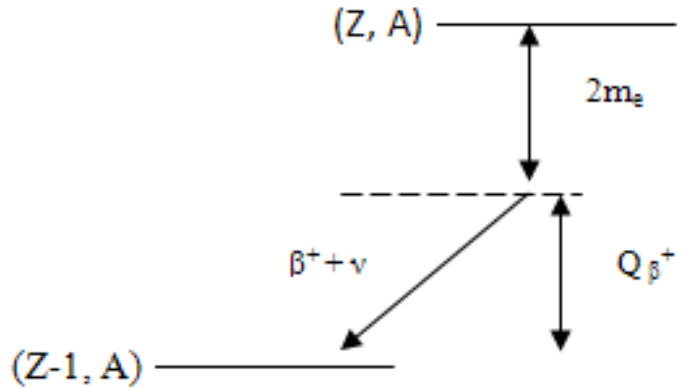

Fig. 1.1 Energy level diagram for $\beta^{+}$decay which will only occur when the mass difference between the parent and daughter nuclide is $>2 m_{e}$. Otherwise only electron capture may occur. 
If the mass-energy condition is satisfied $\beta^{+}$decay is energetically possible. However, in this case electron capture may also occur and competes with $\beta^{+}$decay. In radionuclides with high $\mathrm{Z}$, electron capture predominates while in radionuclides with low Z $\beta^{+}$decay predominates. Work by McFarland et al. parameterizes this phenomenon by demonstrating the $\mathrm{Z}$ dependence in the ratio of the electron capture rate $R_{e}$ to the positron emission rate $R_{\beta+}$ for a wide range of nuclear parameters. It should also be noted that since electron capture and $\beta^{+}$decay are both governed by the Hamiltonian for the week interaction, $R_{e}$ and $\mathrm{R}_{\beta^{+}}$for a given parent radionuclide are related (McFarland, 809-812).

In the case of $\beta^{+}$decay, the resulting positron and the neutrino are ejected from the nucleus with the excess energy beyond the radionuclide's transition energy released during the decay shared between the daughter nucleus, the positron, and the neutrino. Therefore the emitted positron may have a continuum of energies ranging from zero up to a maximum endpoint energy with the mean kinetic energy equal to $1 / 3 \mathrm{E}_{\operatorname{Max} \beta}{ }^{+}$(Bushberg ,595). Using equation 1.7 one can for example calculate the excess free energy available to the resultants of $\beta^{+}$decay of ${ }^{11} \mathrm{C}$ to ${ }^{11} \mathrm{~B}$ :

$$
\mathrm{Q}_{B^{+}}^{11} \mathrm{c}=\left(10,257.08 \frac{\mathrm{MeV}}{\mathrm{c}^{2}}-10,255.1 \frac{\mathrm{MeV}}{\mathrm{c}^{2}}-2\left(.51 \frac{\mathrm{MeV}}{\mathrm{c}^{2}}\right)\right)=.96 \mathrm{MeV}
$$

Table 1.1 lists a number positron emitting radionuclides commonly used in PET and the associated properties of the emitted positron (Prekeges, 191). As expected, $\mathrm{E}_{\operatorname{Max} \beta}{ }^{+}$for ${ }^{11} \mathrm{C}$ is equal to the excess free energy calculated for ${ }^{11} \mathrm{C}$ decay above in the event the positron is imparted with all the energy.

Table 1.1 Positron emitting radionuclides commonly used in PET

\begin{tabular}{|c|c|c|c|c|}
\hline Radionuclide & Half - Life & $E_{\mathrm{Max} \beta^{+}}$ & $\beta^{+}$Max Range in Water & $\beta^{+}$Average Range in Water \\
\hline${ }^{11} \mathrm{C}$ & $20.4 \mathrm{~min}$. & $0.96 \mathrm{MeV}$ & $3.8 \mathrm{~mm}$ & $1.1 \mathrm{~mm}$ \\
\hline${ }^{13} \mathrm{~N}$ & $9.96 \mathrm{~min}$. & $1.19 \mathrm{MeV}$ & $5.0 \mathrm{~mm}$ & $1.5 \mathrm{~mm}$ \\
\hline${ }^{15} \mathrm{O}$ & $123 \mathrm{sec}$. & $1.72 \mathrm{MeV}$ & $8.0 \mathrm{~mm}$ & $2.5 \mathrm{~mm}$ \\
\hline${ }^{18} \mathrm{~F}$ & $110 \mathrm{~min}$. & $0.635 \mathrm{MeV}$ & $2.2 \mathrm{~mm}$ & $0.6 \mathrm{~mm}$ \\
\hline${ }^{68} \mathrm{Ga}$ & $68 \mathrm{~min}$. & $1.9 \mathrm{MeV}$ & $9.0 \mathrm{~mm}$ & $2.9 \mathrm{~mm}$ \\
\hline${ }^{82} \mathrm{Rb}$ & $76 \mathrm{sec}$. & $2.6,3.4 \mathrm{MeV}$ & $15.5 \mathrm{~mm}$ & $5.9 \mathrm{~mm}$ \\
\hline
\end{tabular}

\subsubsection{Annihilation}

Following emission from the nucleus the positron rapidly loses kinetic energy through interactions with surrounding matter; mainly inelastic collisions with atomic electrons. Once most of the positron's kinetic energy is dissipated it will combine with an electron essentially at rest to form a hydrogen like atom known as positronium. With a mean life of between $10^{-7}$ and $10^{-10}$ seconds positronium quickly undergoes a process known as annihilation in which the positron and electron annihilate each other and their mass is converted into electromagnetic energy. In accordance with Einstein's mass-energy relationship $\left(E=m c^{2}\right)$, the annihilation process conserves energy by producing two $511 \mathrm{keV}$ photons emitted simultaneously in opposite directions therefore also conserving momentum. It should be noted that in select cases three photons will be emitted during the annihilation process although this is rare ( $\leq 1 \%$ of events) (Bailey, 21-22).

While the coincidence detection of the two $511 \mathrm{keV}$ photons is the basis for positron emission tomography (PET) there are intrinsic challenges in exploiting this phenomenon owing to the fundamental nature of the annihilation process itself. Namely, the challenges include localizing both the decaying radionuclide, as well as the point of annihilation. First, following emission the positron travels a finite distance before annihilation occurs resulting in localization error of the decaying radionuclide. In PET 
imaging it is the location of the radionuclide itself and not the location of the annihilation event that is desirable. Second, with regards to the two photon annihilation events, many of the photon pairs are not emitted strictly back to back or at $180^{\circ}$ of separation. This is due to the nonzero net momentum of the positron and electron prior to annihilation. This effect is known as noncolinearity and results in a Gaussian distribution of emission angles about $180^{\circ}$ and with a FWHM of $0.5^{\circ}$ (Phelps, 9-12). Once the two coincident photons are detected by the PET system, they are assumed to have originated back to back, thus noncolinearity contributes to the localization error of the annihilation event. Taking both the localization error of the decaying radionuclide and the annihilation event there is a fundamental lower limit to the spatial resolution achievable through PET (Bailey, 23).

\subsection{Fundamentals of Radioactive Decay}

For a single given radionuclide it is impossible to predict the exact temporal instant of decay. However, one may assert that there is a probability that such a decay will occur in a time interval $\mathrm{dt}$ equal to $\lambda \mathrm{dt}$, with $\lambda$, the corresponding decay constant, equal to the probability that the parent radionuclide transitions to the daughter nuclide in one second. From this probabilistic view point it is apparent that given two radionuclides of the same species but of different age the "older" nuclide is no more likely to decay than the "younger" nuclide. Now if one consider a large number $\mathrm{N}$ of radionuclides with $-\mathrm{dN}$ decaying in a time interval dt then the probability any one radionuclide decays in this time interval is - $\mathrm{dN} / \mathrm{N}$ (Reid, 2223). Therefore for a single decay mode we find that:

$$
\frac{d N}{d t}=-\lambda \mathbb{N}
$$

Integrating once yields the solution to equation 1.8 and is known as the radioactive decay law:

$$
N(t)=N_{0} e^{-\lambda t}
$$

with $\mathrm{N}_{0}$ equal to the initial number of parent radionuclides, $\mathrm{N}$ equal to the number of parent radionuclides left after time $t$, and $\lambda$ equal to the decay constant. Regardless of which decay process is being considered all nuclear decays follow this law.

In many cases a parent radionuclide yields a daughter nuclide which is also unstable and prone to decay therefore producing a granddaughter. In this case equation 1.8 must be expanded and modified in order to govern both the growth and decay inherent to sequential decay regimes. In a two sequence decay equation 1.8 becomes:

$$
\frac{\mathrm{dN}_{1}}{\mathrm{dt}}=-\lambda_{1} \mathrm{~N}_{1} \text { and } \frac{\mathrm{dN}_{2}}{\mathrm{dt}}=-\lambda_{2} \mathrm{~N}_{2}+\frac{\mathrm{dN}_{1}}{\mathrm{dt}}
$$

with $\mathrm{N}_{1}$ and $\mathrm{N}_{2}$ respectively equal to the number of patent and daughter radionuclides and $\lambda_{1}$ and $\lambda_{2}$ equal to the corresponding decay constants. The solution to this coupled set of differential equations can be found in the textbook Nuclear Medicine Radiation Dosimetry: Advanced Theoretical Principals (McParland, 155-156).

\subsubsection{Half-Life}

The rate at which a sample of radionuclides decay is most often measured in terms of half-life, $\mathrm{t}_{1 / 2}$, defined as the amount of time it takes for half of the original parent radionuclides to decay. In other 
words after one half-life only half of the original parent radionuclides have survived without decaying. Table 1.1 lists the half-life of some of the positron emitting radionuclides most commonly used in PET. In terms of $\lambda$ half-life is defined as:

$$
t_{1 / 2}=\frac{\ln 2}{\lambda}=\frac{0.693}{\lambda}
$$

In a clinical setting, one must consider the effective half-life of a radionuclide. The effective halflife of a radionuclide is the sum of the physical half-life and the biological half-life. The biological halflife stems from the body's physiological response to excrete foreign substances, for example injected radioactivity, and must be accounted for when imaging patients.

\subsubsection{Activity}

Also of prime importance to PET is the activity of a sample of radionuclides. A low activity source may be difficult to detect or require an extended period of scanning time in order to gather sufficient statistics while a high activity source may lead to increased noise as well as saturation within the PET scanner electronics ultimately resulting in a decrease in spatial resolution of the scanner (further discussed in Chapter 2). Of course in a clinical setting a high activity source is also of great concern with regards to both clinician and patient safety. Activity is defined as the absolute value of equation 1.8:

$$
\text { Activity }=\left|\frac{d N}{d t}\right|=\lambda N_{0} e^{-\lambda t}=\lambda N
$$

Activity is expressed in SI units of number of decays per second with:

$$
1 \text { becquerel }(\mathrm{Bq})=1 \text { decay per second }
$$

Activity is also expressed in curies with:

$$
1 \text { curie }(\mathrm{Ci})=3.7 \times 10^{10} \text { becquerel }(\mathrm{Bq})
$$

\subsection{Photon Interactions in Matter}

Post positron electron annihilation, it is important to understand how the resultant $511 \mathrm{keV}$ photons interact with matter. In PET it is desirable to detect coincidence $511 \mathrm{keV}$ photons that have been unimpeded post-annihilation although this is not always the case. Annihilation photons that have been scattered prior to being detected, i.e. depositing their energy in the PET scanner, can lead to degraded spatial and energy resolution as well as decreased scanner sensitivity. The two primary mechanisms for matter interaction in $511 \mathrm{keV}$ PET are the photoelectric effect, which predominates at incident photon energies $\leq 100 \mathrm{keV}$, and Compton scattering, which predominates at incident photon energies ranging from $100 \mathrm{keV}$ to $\sim 2 \mathrm{MeV}$. There is also a third mechanism known as pair production which predominates at incident photon energies $\geq 1.022 \mathrm{MeV}$ and as a result is of little consequence with regards to $511 \mathrm{keV}$ PET and will not be expanded upon further (Bailey, 24-25).

\section{$\underline{\text { 1.5.1 Photoelectric Effect }}$}

The photoelectric effect (Fig. 1.2a) occurs when an incident photon transfers all of its energy $E_{p}$ to a bound orbital electron (primarily in the inner shells) of an absorbing material. Assuming the incident 
photon energy $E_{p}$ is greater than the electron binding energy $E_{B}$, the electron, known as a photoelectron, is ejected from the atom and has energy $\mathrm{E}_{\mathrm{P}}-\mathrm{E}_{\mathrm{B}}$. Consequently a loosely bound higher orbital electron drops down to fill the lower orbital vacancy and in the process emits a characteristic x-ray of energy equal to the energy difference between orbital states. In some cases the absorbing atom may eject a second electron, know as an Auger electron, in order to relieve the excess energy.

The probability of the photoelectric effect occurring decreases with increased incident photon energy. Therefore, in the range of photon energies desirable for PET $(\sim 511 \mathrm{keV})$, the photoelectric effect only plays a minor role in the attenuation (see section 1.5.3) of incident annihilation photons and usually only occurs following one or more Compton scatter events.

(a)

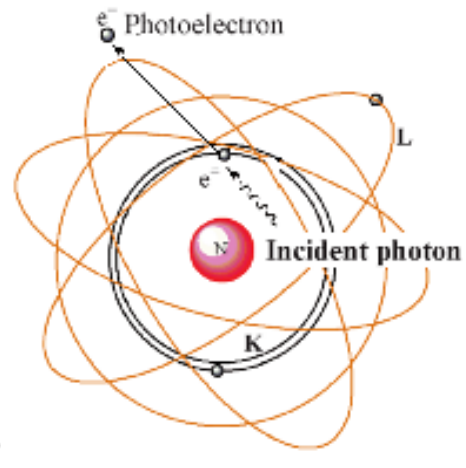

(b)

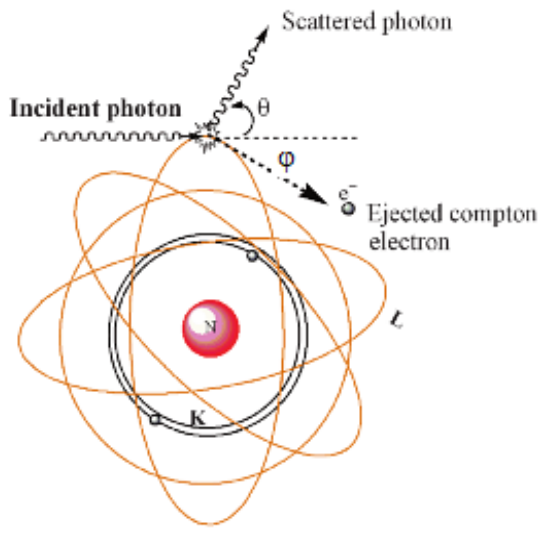

Fig 1.2 (a) Incident photons interacting with bound electrons of an absorber resulting in the photoelectric effect and (b) Compton scatter (Vallabhajosula, 61).

\subsubsection{Compton Scattering}

Compton scattering (Fig. 1.2b) occurs when an incident photon of energy $E_{0 \mathrm{P}}$ transfers only a fraction of its energy $\mathrm{E}_{\mathrm{p}}$ to a bound orbital electron (primarily in the outer shells) of an absorbing material. The outcome of this inelastic collision is an ejected electron with energy $E_{e}$, known as a Compton electron, as well as a scattered photon of energy $E_{p}$. Overall, $E_{p}{ }^{`}=E_{B}+E_{e}+E_{p}$. Utilizing conservation laws one can determine the energy $E_{p}$ of the scattered photon as a function of the scatter angle $\theta$ (Griffiths, 514-515). First, consider the conservation of momentum between the scattered photon and the ejected Compton electron in the vertical direction. The momentum of the scattered photon is $p_{p} \sin (\theta)$ and that of the ejected electron is $\mathrm{p}_{\mathrm{e}} \sin (\varphi)$ and since $\mathrm{p}_{\mathrm{P}}=\mathrm{E}_{\mathrm{P}} / \mathrm{c}$ conservation of momentum gives:

$$
\sin (\phi)=\frac{E_{p}}{p_{e} c} \sin (\theta)
$$

Conservation of momentum in the horizontal direction gives:

$$
\frac{E_{0 P}}{c}=p_{p} \cos (\theta)+p_{e} \cos (\phi)=\frac{B_{p}}{c} \cos (\theta)+p_{e}\left(1-\left(\frac{E_{p}}{p_{e} c} \sin (\theta)\right)^{2}\right)^{1 / 2}
$$

Squaring both sides of equation 1.15 and reducing yields:

$$
\mathrm{p}_{e}{ }^{2} \mathrm{c}^{2}=\left(\mathrm{B}_{0 \mathrm{P}}-\mathrm{B}_{\mathrm{p}} \cos (\theta)\right)^{2}+\mathrm{B}_{\mathrm{p}}{ }^{2} \sin ^{2} \theta=\mathrm{B}_{0 \mathrm{p}}{ }^{2}-2 \mathrm{~B}_{0} \mathrm{P} \mathrm{B}_{\mathrm{p}} \cos (\theta)+\mathrm{B}_{\mathrm{p}}{ }^{2}
$$


Next, with $m_{e}$ equal to the mass of the electron and $E_{e}$ equal to the relativistic energy of the electron, conservation of energy dictates that:

$$
\mathrm{B}_{0 \mathrm{P}}+\mathrm{m}_{e} \mathrm{c}^{2}=\mathrm{B}_{\mathrm{p}}+\mathrm{B}_{e}=\mathrm{B}_{\mathrm{p}}+\left(\mathrm{me}_{e}{ }^{2} \mathrm{c}^{4}+\mathrm{p}_{e}{ }^{2} \mathrm{c}^{2}\right)^{1 / 2}
$$

Finally, substituting equation 1.16 into 1.17 and solving for $E_{p}$ yields the desired relationship between the scatter angle and energy of the scattered photon:

$$
\mathrm{E}_{\mathrm{p}}=\frac{\mathrm{m}_{\mathrm{e}} \mathrm{c}^{2}}{1-\cos (\theta)+\frac{\mathrm{m}_{\mathrm{e}} c^{2}}{\mathrm{E}_{0 \mathrm{P}}}}
$$

When only considering $511 \mathrm{keV}$ photons equation 1.18 simplifies further to:

$$
\mathrm{E}_{\mathrm{p}}=\frac{511 \mathrm{keV}}{2-\cos (\theta)}
$$

This relationship demonstrates that even with minimal loss of energy, a scattered photon may drastically change direction. This can be especially problematic in PET as the scattered photon may still fall within the energy acceptance window of the scanner and thus be interpreted as part of a true back to back coincidence event when in fact it was part of a scattered coincidence. With Compton scatter being responsible for the majority of scatter events in PET, this error can lead to appreciable decreases in spatial resolution as well as image blur and must, therefore, be minimized. Such minimization may be accomplished through detector geometry and design, narrowing the energy acceptance window, and/or utilizing scatter and attenuation correction algorithms.

\subsubsection{Attenuation}

As photons encounter matter they are either transmitted through the material without interaction or scattered. Scattering along with the photoelectric effect causes the attenuation of a beam of photons incident on an absorbing material as illustrated in Fig 1.3.

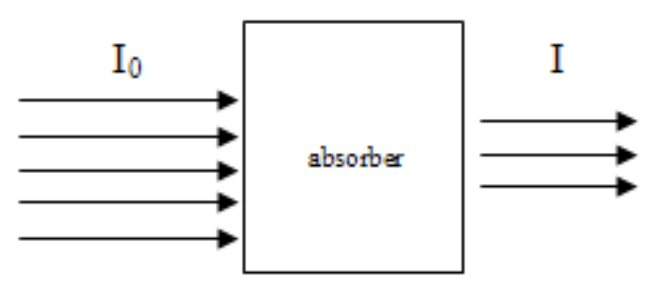

Fig 1.3 Exponential attenuation of an incident photon beam $\mathrm{I}_{0}$ on an absorbing material of thickness $\mathrm{dx}$ and with linear attenuation coefficient $\mu$.

Consider a beam of $\mathrm{I}_{0}$ photons incident on an absorbing material of thickness $\mathrm{dx}$ with linear attenuation coefficient $\mu$ equal to the probability per unit distance that an interaction will occur. The attrition of photons from the beam is thus $\mathrm{dI}_{0}$ with:

$$
d I_{0}=-\mu I_{0} d x
$$

Integrating once yields the solution, known as Beer's Law, to equation 1.20, and demonstrates an exponential decrease in intensity of a photon beam as it propagates through an absorber (Fox, 4-5): 


$$
\mathrm{I}=\mathrm{I}_{0} \mathrm{e}^{(-\mu x)}
$$

Attenuation is a function of photon energy as well as the electron density of the absorber and thus $\mu$ typically decreases with increased incident photon energy and increases with atomic number and density of the absorber. In order to account for density differences between absorbers of the same composition the mass attenuation coefficient is used and is defined as:

$$
\text { Mass att. coefficient }=\frac{\mu}{\rho}
$$

with $\rho$ equal to the density of the absorber.

In PET, $\mu / \rho$ is approximated as the sum of mass attenuation coefficients corresponding to both Compton scatter and the photoelectric effect within an absorber as they are the two predominate causes of attenuation. Typical values for the mass attenuation coefficient in absorbers commonly encountered in PET, for example, tissue (soft and hard), bone, tungsten, lead, and various scintillation materials can be found on the National Institute for Standards and Technology website (Hubbell, 1996). The tables list the mass attenuation coefficients for photon energies from $1 \mathrm{keV}$ to $20 \mathrm{MeV}$.

It should also be noted that one advantage of PET is that attenuation is only a function of the thickness of the absorbing material and not a function of the distance traveled in the absorber. This can be demonstrated by consider two collinear photon beams originating at the center of an absorber of thickness $\mathrm{x}$ and propagating outward along the $\mathrm{x}$-axis towards photodetectors at each end. The sum attenuation of the photon beams will thus be:

$$
I=I_{0}^{1} e^{\left(-\frac{\mu x}{2}\right)} * I_{0}^{2} e^{\left(-\frac{\mu \pi}{2}\right)}=I_{0} e^{-\mu\left(\frac{\pi}{2}+\frac{\pi}{2}\right)}=I_{0} e^{-\mu x}
$$

\subsection{Overview}

All matter is comprised of constituent atoms, some of which are unstable and prone to radioactive decay. Those unstable atoms are known as radionuclides. Radionuclides which decay by positron emission $\left(\beta^{+}\right.$ decay) produce two collinear or nearly collinear $511 \mathrm{keV}$ annihilation photons which are the foundation for positron emission tomography (PET). Prior to detection radiation may interact with matter with the photoelectric effect and Compton scatter being the two primary interaction mechanisms for $511 \mathrm{keV}$ PET.

In order to further understand how PET exploits the natural phenomenon of radioactive decay and radiation interaction in matter the next chapter will focus on scintillation detectors. Scintillation detectors are integral to almost all PET system as they are the first step in converting incident ionizing radiation into an electrical current.

\section{References}

Bailey, Dale L., David W. Townsend, Peter E. Valk, and Michael N. Maisey, eds. Positron Emission Tomography: Basic Sciences. London: Springer-Verlang London Limited, 2005.

Bushberg, Jerrold T., J. Anthony Seibert, Edwin M. Leidholdt, Jr., and John M. Boone, The Essential Physics of Medical Imaging. $2^{\text {nd }}$ ed. Philadelphia: Lippincott Williams \& Wilkins: 2002.

Fox, Mark, Optical Properties of Solids. New York: Oxford University Press Inc., 2010. 
Griffiths, David J., Introduction to Electrodynamics. $3^{\text {rd }}$ ed. Upper Saddle River: Prentice-Hall, Inc., 1999.

Hubbell, J.H. and S.M. Seltzer, "Tables of X-Ray Mass Attenuation Coefficients and Mass Energy-Absorption Coefficients (version 1.4).” [Online] Available: http://physics.nist.gov/xaamdi [2011, May 1]. National Institute of Standards and Technology, Gaithersburg, MD, 2004.

McFarland, W. B., and W. W. Repko, "Ratio of electron capture to positron emission in the elementary-particle treatment of nuclear $\beta$ decay.” Physics Review C, vol. 15, no. 2, pp. 809-812, 1977.

McParland, Brian J., Nuclear Medicine Radiation Dosimetry: Advanced Theoretical Principal. London: SpringerVerlang London Limited, 2010.

Phelps, Michael E., ed. PET: Physics Instrumentation, and Scanners. New York: Springer Science + Business Media, Inc., 2006.

Prekeges, Jennifer, Nuclear Medicine Instrumentation. Sudbury: Jones and Bartlett Publishers, LLC, 2011.

Reid, J. M., The Atomic Nucleus. Manchester: Manchester University Press, 1984.

Saha, Gopal B., Basics of PET Imagine: Physics, Chemistry, and Regulations. New York: Springer Science + Business Media, Inc., 2005.

Vallabhajosula, Shankar, Molecular Imaging: Radiopharmaceuticals for PET and SPECT. Heidelberg: SpringerVerlang Berlin Heidelberg, 2009.

Williams, W.S.C., Nuclear and Particle Physics. New York: Oxford University Press Inc., 1991. 


\section{2 Scintillation Detector Principals and Properties}

\section{$\underline{2.1 \text { Introduction }}$}

The most common method for detecting $511 \mathrm{keV}$ photons in PET imaging is through the use of scintillation detectors (Bailey, 30). Such detectors absorb the energy of incident $511 \mathrm{keV}$ photons (photoelectric effect or Compton scatter) and as a result isotropically emit scintillation photons from the point of interaction through a process known as luminescence, or more specifically, prompt florescence.

Scintillators can be either organic or inorganic and come in a solid or liquid form. Organic scintillators tend to have faster decay times than inorganic scintillators while inorganics tend to have increased light yield and linearity than organic scintillators. However, while increased light yield and linearity are desirable it is the increased stopping power of solid inorganic scintillators that make them the most desirable for detecting $511 \mathrm{keV}$ photons. As such, only solid inorganic scintillators will be discussed further.

In this chapter the scintillation process and consequently the band structure of scintillators will be discussed. The characteristic properties of scintillation detectors and their applicability to PET will also be presented.

\subsection{Organic Scintillator Band Structure and the Scintillation Process}

Within a crystal lattice, the once discrete outer orbital electronic energy levels associated with an isolated atom become broadened into a series of allowed bands due to the overlap of the outer orbitals of neighboring atoms. Each band can contain $2 \mathrm{~N}$ electrons, with $\mathrm{N}$ equal to the number of primitive unit cells in the crystal. Electrons fill the allowed bands up to the Fermi energy $E_{F}$ which is determined by the electron density of the crystal (Fox, 271).

Scintillators are composed of either materials classified as insulators or semiconductors. These types of material have an even number of electrons per atom and therefore the highest occupied band is full. The last filled band is known as the valance band while the first unoccupied band is known as the conduction band. Electrons in the valance band are essentially bound to the lattice while those in the conduction band are free to migrate throughout the crystal (Knoll, 255). Separating the valance and conduction bands are forbidden bands which an electron may not occupy. These forbidden bands constitute the band gap with energy $\mathrm{E}_{\mathrm{g}}$. As a result of the last occupied band being full the Fermi energy resides within the band gap (Fig 2.1).

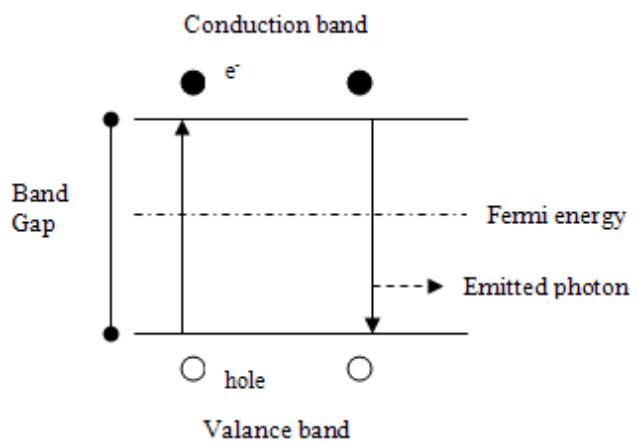

Fig 2.1 Example band structure of a pure scintillator with valance band electron excitation. 
While valance electrons are intrinsically bound to the lattice, they can absorb energy, for example by interaction with an incident $511 \mathrm{keV}$ photon, and be excited across the band gap into the conduction band (Fig 2.1).

Prior to de-excitation the excited electron is free to migrate throughout the crystal. In doing so the free electron may ionize atoms within the lattice resulting in secondary ionization events and the liberation of a second electron from the valance band. Both electrons may continue to ionize further atoms in the lattice ultimately resulting in a domino effect and a cascade in electron-hole pair production. Ultimately the electrons lose energy through inelastic collisions and scatter, ceasing further ionization events. The characteristic duration of this ionization/relaxation process is typically between $10^{-15}$ and $10^{-13}$ seconds (Weber, 37) after which the excited electrons de-excite and return to their ground state in the valance band. It is the de-excitation of the electrons back down to the valance band that produces scintillation photons with energy equal to $E_{g}=h c \backslash \lambda$ with $h$ equal to Plank's constant, $\mathrm{c}$ equal to the speed of light, and $\lambda$ equal to the wavelength of the photon.

However, in many instances $E_{g}$ is such that the emitted photon's wavelength will be in the ultraviolet range of the spectrum. This is problematic as a majority of commercially available photodetectors operate in the visible and near infrared spectrum. For example, silicon, used in avalanche photodiodes (APDs) as well as newly developed silicon photomultipliers (SiPMs), has an operational excitation (absorption) spectrum of 400-1100nm. Beyond this wavelength sensitivity range the responsivity of silicon falls off drastically (Keiser, 109-114).

Therefore, in order to overcome this problem, impurities are added to the pure scintillator causing the band structure to be modified. The impurities, known as [luminescence] activators, create new allowed energy bands within the band gap: an activator ground state and activator excited states (Fig 2.2).

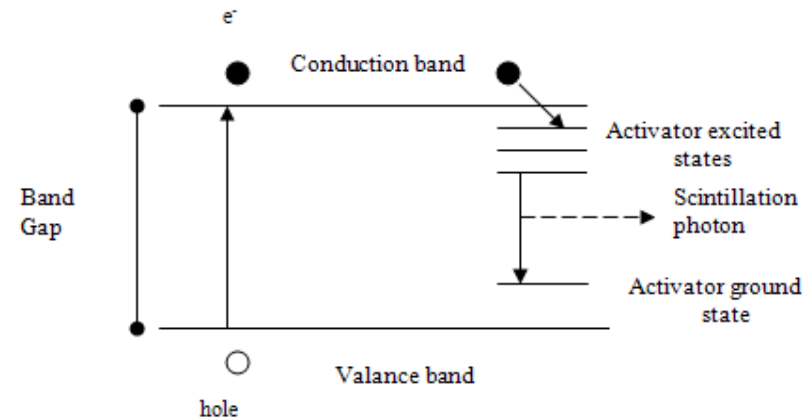

Fig 2.2 Example band structure an activated or doped scintillator. Activator states reside within the traditionally forbidden band gap.

As electrons and holes are being produced by the process described above the positive holes will quickly migrate to the activator site and ionize it. This is due to the fact that the ionization energy of the activator will be less than that of constituent lattice atoms (Knoll, 255). As described above the electrons migrate throughout the lattice but instead of de-exciting back to the valance band they may be captured and drop into one of the excited states of the ionized activators located in the band gap just below the normal conduction band. This results in a neutral, although, excited configuration of the activator with a typical half-life on the order of $0.1 \mu$ seconds (McParland, 387). If there is a transition between the excited state and the ground state of the activator the electrons will de-excite and emit a photon in the process. As the de-excitation occurred within the band gap the emitted photon will have energy $<\mathrm{E}_{\mathrm{g}}$ and therefore lie within the visible spectrum.

The number of scintillation photons $\mathrm{N}_{\mathrm{ph}}$ produced by the absorption of incident ionizing radiation 
with energy $\mathrm{E}$ is given by:

$$
\mathrm{N}_{\mathrm{ph}}=\frac{\mathrm{E}}{\beta \mathrm{E}_{\mathrm{gap}}} \mathrm{SO}
$$

with $\beta$ equal to the conversion efficiency for creating one thermalized electron-hole pair, $E_{\text {gap }}$ equal to the band gap energy, S equal to the transfer/transport efficiency of electron and holes to luminescence activators, and Q equal to radiative efficiency of the excited luminescence activator. Of all the parameters in equation 2.0, the transfer/transport efficiency $\mathrm{S}$ has the highest rate of variability. This is because $\mathrm{S}$ depends on defects present in the scintillator aside from the doped activators. The excess defects may capture the thermalized electrons and or holes prior to being captured by an activator resulting in quenching in the scintillator (van Eijk, 2002, R96). (The quantity $\mathrm{N}_{\mathrm{ph}} / \mathrm{E}$ is known as light yield and discussed in the Characteristic Properties section).

\subsubsection{Quenching}

Quenching is any nonradiative transition of thermalized electrons resulting in no scintillation photons being produced. Quenching occurs due to impurities within the scintillator (as discussed above), as well as select nonradiative transitions between activator excited states and the activator ground state. In both cases quenching represents a loss mechanism in converting incident ionizing radiation into scintillation photons. There are various modes of quenching (Ahmed, 327-328), however, each reduces the efficacy and performance of a scintillator.

\subsubsection{Afterglow}

Afterglow is simply undesirable background light in a scintillator. The two main sources of afterglow include phosphorescence and delayed florescence.

Phosphorescence usually occurs when a migrating thermalized electron in the conduction band is captured by an ionized activator but falls into an activator excited state for which there is no allowed transition down to the activator ground state. Therefore, the electron requires excess energy to be excited up to an activator excited state for which there is an allowed transition down to the activator ground state. Thermal energy is the main source of the required excess energy and consequently as temperature of the scintillator increases afterglow increases.

Delayed Florescence usually occurs when there is an increase in the amount of time required to transfer/transport a thermalized electron and or hole to an activator site. While impurities within the scintillator may capture thermalized electrons and or holes in route to an activator site they may also scatter them. This results in a delayed arrival to an activator site and thus a delayed emission of scintillation photons upon relaxation.

Both phosphorescence and delayed florescence result in an increased relaxation time following electron ionization. As a result, scintillation photons are produced which either artificially broaden a light pulse corresponding to an ionization event, or contribute to the background afterglow of the scintillator. In either case, light yield and position and energy resolution may be deleteriously affected.

\section{$\underline{\text { 2.3 Characteristic Properties }}$}

There are seven characteristic properties of scintillation detectors that must be considered when determining their applicability for applications in PET. These properties include; stopping power of 
$511 \mathrm{keV}$ photons, decay time, light yield per keV of photon energy deposited, intrinsic energy resolution, transparency to the wavelength of its own emission, linearity, and cost/practicality.

\subsubsection{Stopping Power}

Stopping power of a scintillator is defined as the mean distance an incident photon travels within the scintillation crystal before completely depositing its energy and coming to rest. Stopping power is characterized by the attenuation length (the length over which the intensity of an incident photon beam has dropped to $\mathrm{e}^{-1}$ ) and from Eq. 1.21 is equal to $1 / \mu$ with $\mu$ equal to the linear attenuation coefficient of the scintillation material. The linear attenuation coefficient depends on both the density and effective atomic number of the scintillation material and has units of inverse length. Scintillators with shorter attenuations lengths are therefore more efficient per unit length at stopping incident $511 \mathrm{keV}$ photons as compared to scintillators with longer attenuations lengths. For example, at $511 \mathrm{keV}$ the attenuation length for bismuth germanate (BGO) is $1.04 \mathrm{~cm}$ while for lanthanum bromide $\left(\mathrm{LaBr}_{3}\right)$ it is $2.13 \mathrm{~cm}$ (Zaidi, 15). Therefore, in order to have equivalent detection efficiencies, $\mathrm{LaBr}_{3}$ scintillators must be more than twice as thick as BGO scintillators.

\subsubsection{Decay Time}

Decay time is the amount of time elapsed between the excitation of a bound valance electron within the crystalline lattice of the scintillator to a higher energy state and the subsequent decay of the excited electron back down to the ground state and emission of a photon. It is the decay time between these states that determines the time characteristic of the emitted scintillation light (Knoll, 256). On a single electron by electron basis the radiative lifetime $\tau_{\mathrm{R}}$ (the lifetime of an excited electron independent of nonradiative transition) of an allowed electric-dipole transition between states $i$ and $j$ is given by:

$$
\frac{1}{\tau_{R_{i} j}}=\frac{n(n+2)^{2}}{9} \frac{e^{2} f_{i j}}{2 \pi \epsilon_{0} m c \lambda^{2}}
$$

with $\mathrm{n}$ equal to the refractive index, e equal to the electron charge, $\mathrm{f}_{i j}$ equal to the oscillator strength, $\varepsilon_{0}$ equal to the permittivity of free space , m equal to the mass of the electron , c equal to the speed of light, and $\lambda$ equal to the emission wavelength (Weber, 40). It should however be noted that the measured decay time for a scintillator is not a direct measure of the radiative lifetime but instead the measure of an effective lifetime such that $\tau_{\text {eff }}$ is a combination of both radiative $\left(\tau_{\mathrm{R}}\right)$ and nonradiative $\left(\tau_{\mathrm{NR}}\right)$ lifetimes (Woo, 1154):

Scintillator decay times are typically expressed in nanoseconds and the shorter the decay time the more efficiently the scintillator can produce individual pules at high count rates. As a result, fast decay times also reduce the number of random coincidences within a PET geometry.

\subsubsection{Light Yield}

Light yield is defined as the number of scintillation photons produced per $\mathrm{keV}$ of deposited energy and is given by multiplying each side of equation 2.0 by $1 / E$ with $E$ equal to the energy of the incident ionizing radiation. The fraction of incident ionizing radiation energy that is converted into visible light is known as the quantum efficiency $(\eta)$ of the scintillation detector and is given by:

$$
\eta=\beta \mathrm{SO}
$$


with $\beta$ equal to the conversion efficiency for creating thermalized electron-hole pairs, $S$ equal to the transfer/transport efficiency of electron and holes to luminescence activators, and Q equal to the radiative efficiency of luminescence activators.

When discussing light yield one must take care to distinguish between absolute light yield and effective light yield (the number of scintillation photons collected by an attached photodetector). Effective light yield is proportional to the light collection efficiency of a scintillation detector which is a function of crystal size and shape, surface finish, refractive index, and the properties of any reflector material used. Work done by Moszyński et al. demonstrates that in order to obtain the absolute light yield a correction term must be applied to the effective light yield. This is done by measuring the effective light yield the distance between the point of scintillation and an attached photodetector (depth of interaction). The data is plotted and the associated fit extrapolated to zero thickness corresponds to the absolute light yield (Moszyński, 1053-1058).

There are however fluctuations in the number of scintillation photons detected. These fluctuations are governed by Poisson counting statistics and decrease as $\mathrm{N}^{-1 / 2}$ with $\mathrm{N}$ equal to the number of scintillation photons detected (Phelps, 18).

For each scintillation event there is a single corresponding light pulse detected by an associated photodetector with the amplitude of the pulse proportional to the effective light yield. The light pulse is converted to an electrical current by the photodetector and collected over a variable time $t$ known as the gate width. The time integral over the duration of the current equals the total charge generated and is a direct metric (neglecting photodetector inefficiencies) for the amount of energy deposited in the scintillator. High light yield scintillators such as cerium doped yttrium oxyorthosilicate (YSO:Ce) and thallium doped sodium iodide (NaI:Tl) (46 and 38 photons/keV respectively) (Saha, 20) statistically produced more well defined light pulses therefore resulting in less error and uncertainty in the amount of energy deposited. This metric can be used in energy resolution calculations as well as depth of interaction calculations when the light pulse is viewed from two adjacent ends of the scintillator.

\subsubsection{Intrinsic Energy Resolution}

When dealing with multiple scintillation events, light yield will fluctuate on an event by event basis. Intrinsic catalyst for these fluctuations within the scintillator include: Poisson counting statistics discussed above, impurities and inhomogeneities within the scintillator resulting in either an increased electron-hole recombination rate prior to scintillation photons being produced and or the scatter of scintillation photons that are produced, and temperature variations of the scintillator. (As temperature increases the thermal recombination rate increases, thus resulting in decreases in light yield.) It should be noted that vicissitudes in the energy deposited for each scintillation event due to scatter of the incident radiation prior to interacting with the scintillator are also sources of fluctuation in light yield but are not inherent to the scintillator itself.

As a result of the fluctuations in light yield there is a distribution in the measured energy deposited in the scintillator. This distribution is most commonly displayed in a histogram with the vertical axis equal to the number of counts and the horizontal axis equal to energy and partitioned into bins, or amplitude increments, of size dE (Fig 2.3).

Energy resolution is defined as the full-width-half-maximum (FWHM) of the measured energy distribution divided by the centroid of the distribution. Energy resolution is therefore a dimensionless quantity and usually expressed in percent. 
The intrinsic energy resolution of a scintillator, coupled with the intrinsic energy resolution of the associated photodetector, are important because sufficiently high energy resolution allows for the efficient rejection of background radiation, as well as low energy $(<511 \mathrm{keV})$ incident gammas that have been Compton scattered prior to entering the scintillator. This discrimination process is achieved using a pulse high analyzer and placing a lower limit threshold on the total charge generated by each event. As the threshold is raised energy resolution will increase but at the expense of detector efficiency.

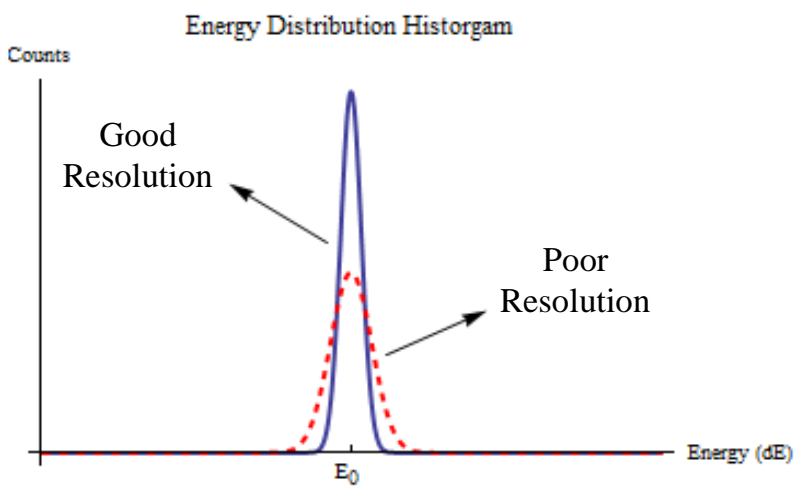

Fig 2.3 Example of an energy distribution histogram demonstrating relatively good (solid blue) and poor (red dashed) energy resolutions.

\subsubsection{Transparency}

Transparency of a scintillator to the wavelength of the scintillation photons it produces is paramount to scintillator performance. In the case of pure "non-activated" scintillators the absorption and emission spectrums overlap as roughly the same amount of energy required to excite a valance electron to the conduction band is emitted upon electron de-excitation. This leads to substantial optical self-absorption within the scintillator thus leading to deleterious effects on light yield, energy resolution, and position resolution.

While the motivation behind using activator impurities is discusses above, one latent and beneficial consequence of luminescence through such impurities is the mitigation of optical selfabsorption. This is achieved as the energy associated with the de-excitation of an electron from an activator excited state down to the activator ground state is less than the energy required to excite the electron up into the conduction band. Consequently the emission spectrum is shifted to longer wavelengths and will not overlap the absorption spectrum. The emission spectrum for scintillators commonly used in PET can be found in the text by Wernick et al. (Wernick, 236) while the emission spectrum for newly developed scintillators such as cerium doped lutetium yttrium oxyorthosilicate (LYSO:Ce) (Pepin, 789-795) can be found on the IEEE digital database.

\subsubsection{Linearity}

Linearity of a scintillator refers to the proportionality of light yield to the amount of energy deposited by incident ionizing radiation. In an ideal scintillator it is desirable for this proportionality to be linear over the entire energy spectrum. Scintillator linearity is tested by measuring the Compton edge energy for a range of incident ionizing radiation energys and plotted against the histogram energy bin to which the event corresponds. An idealized example of such a test is showing in Figure 2.4 below (Alfassi, 293). 


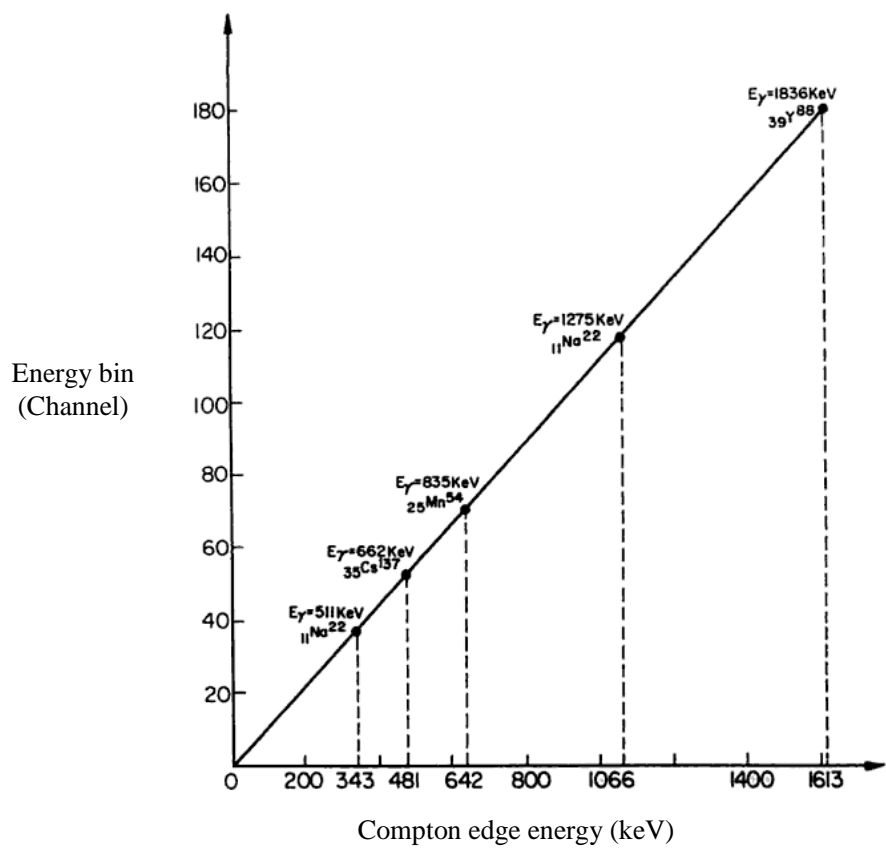

Fig 2.4 Idealized example for scintillator response linearity.

Linearity of response is of particular importance to energy resolution as nonlinear behavior will lead to discontinuities in light yield and thus degraded energy resolution. Nonlinearities can arise from nonradiative and quenching effects as well as saturation.

\section{$\underline{\text { Saturation }}$}

Saturation is defined as the point at which the rate of excited electrons and holes captured by activator sites is in equilibrium with the rate of excited electron de-excitation. Saturation may also be viewed as the maximum excited electron density for which the number of available unoccupied ionized activator sites approaches zero. Consequently, saturation is a function of the activity rate incident on scintillator.

According to Murray et al. (Murray, 815-826) the depletion of available activator sites resulting from incident radiation with a high ionization density $(\mathrm{dE} / \mathrm{dx}$ ) can also account for observable saturation effects. Consequently scintillator saturation is not only activity rate dependent but also radiation species specific. The relationship between light yield and saturation is parameterized by the Birks formula (Lecoq, 3.1.1-15):

$$
\mathrm{N}_{\mathrm{ph}}=\frac{\mathrm{N}_{\mathrm{ph}}}{1+\mathrm{a}_{\mathrm{B}} \frac{\mathrm{dE}}{\mathrm{dx}}}
$$

with $\mathrm{N}_{\mathrm{ph}}$ equal to the number of scintillation photons produced in the presence of saturation effects, $\mathrm{N}_{\mathrm{ph}}$ equal to the number of scintillation photons produced in the absence of saturation, $\mathrm{a}_{\mathrm{B}}$ equal to the Birks parameter, and $\mathrm{dE} / \mathrm{dx}$ equal to the ionization density of the incident radiation.

\subsubsection{Practicality and Cost}

While a scintillator may be theoretically optimal and or viable on a small scale, modern high resolution PET scanners require scintillators which are rugged and cost efficient, and able to be produced on a large enough scale to be of practical interest. For example, LSO:Ce, while appearing to be one of the best scintillators for PET applications (Kimble, 1434), as well as LYSO:Ce, have not been as commercial 
utilized, for example, as BGO because of: 1 . their high cost of $\sim \$ 50 / \mathrm{cm}^{3}$ due to the expense of Lu (quote: Proteus, Inc., Sept. 2011), 2. manufacturing difficulty, and 3. the presence of the naturally occurring radioactive isotope ${ }^{176} \mathrm{Lu}$ which produces two prominent gamma rays of 201 and $306 \mathrm{keV}$ (van Eijk, 2002, R101-R102; Humm, 1581).

\section{$\underline{2.4 \text { Overview }}$}

The use of solid inorganic scintillators is the most common and efficient method for detecting $511 \mathrm{keV}$ photons utilized in PET imaging. Incident ionizing radiation is detected via the utilization of inorganic scintillator band structure and the generation scintillation photons. The band structure of scintillators may be manipulated by the addition of [luminescence] activators resulting in a more efficient scintillation process as well as visible spectrum photons.

When considering a scintillator for PET applications there are a number of characteristic properties that much be considered. No scintillator will simultaneously meet all the optimized parameters among the characteristic properties and therefore choosing the appropriate scintillator for a given application is always an exercise in compromise. A more in depth analysis and overview of scintillators typically used in current PET applications can be found in the review article by Humm et al. (Humm, 1580-1582). However, there are new scintillators being developed and tested, such as cerium doped lanthanum bromide $\left(\mathrm{LaBr}_{3}: \mathrm{Ce}\right)$ and lutetium iodide $\left(\mathrm{LuI}_{3}: \mathrm{Ce}\right)$ (van Eijk, 2006, 259-274) both of which show encouraging prospects for use in PET.

As mentioned in this chapter, scintillators are coupled to one or more photodetectors used to convert the generated scintillation photons into an electrical pulse. The next chapter will therefore examine the fundamental operation and design of photodetectors with the main focus on solid state photodetectors; specifically the newly developed silicon photomultiplier.

\section{$\underline{\text { References }}$}

Ahmed, Syed Naeem, Physics \& Engineering of Radiation Detection, London: Academic Press, 2007.

Alfassi, Zeev B., Activation Analysis, Volume II. Boca Raton: CRC Press, 1990.

Bailey, Dale L., David W. Townsend, Peter E. Valk, and Michael N. Maisey, eds. Positron Emission Tomography: Basic Sciences. London: Springer-Verlang London Limited, 2005.

Fox, Mark, Optical Properties of Solids. New York: Oxford University Press Inc., 2010.

Humm, John L., Anatoly Rosenfeld, and Alberto Del Guerra, "From PET detectors to PET scanners.” European Journal of Nuclear Medicine and Molecular Imaging, vol. 30, no. 11, pp. 1574-1597, 2003.

Keiser, Gerd, Optical Communication Essentials. New York: McGraw-Hill Professional Publishing, 2003.

Kimble, Thomas, Mitch Chou, and Bruce H. T. Chai, "Scintillation Properties of LYSO Crystals." 2002 IEEE Nuclear Science Symposium Conference Record, vol. 3, pp.1434-1437, 2003.

Knoll, Glenn F., Radiation Detection and Measurement. New York: John Wiley \& Sons, Inc., 1979.

Lecoq, P., "Scintillation Detectors for Charged Particles and Photons." Numerical Data and Functional Relationships in Science and Technology: N.s. Group 1. Vol. 21. Subvol. B. Pt. 1. C. W. Fabjan and H. Schopper, eds. New York: Springer, 2011, 3.1.1-1-27. 
McParland, Brian J., Nuclear Medicine Radiation Dosimetry: Advanced Theoretical Principal. London: SpringerVerlang London Limited, 2010.

Moszyński, M., M. Kapusta, M. Mayhugh, D. Wolski, and S.O. Flyckt, “Absolute Light Output of Scintillators.” IEEE Transactions on Nuclear Science, vol. 44, no. 3, pp. 1052-1061, 1997.

Murray, R. B., and A. Meyer, "Scintillation Response of Activated Inorganic Crystals to Various Charged Particles.” Physics Review, vol. 122, issue 3, pp. 815-826, 1961.

Pepin, Catherine Michelle, Philippe Bèrard, Anne-Laure Perrot, Claude Pèpin, Daniel Houde, Roger Lecomte, Charles L. Melcher, and Herni Dautet, "Properties of LYSO and Recent LSO Scintillators for Phoswich PET Detectors.” IEEE Transactions on Nuclear Science, vol. 51, no. 3, pp. 789-795, 2004.

Phelps, Michael E., ed. PET: Physics Instrumentation, and Scanners. New York: Springer Science + Business Media, Inc., 2006.

Saha, Gopal B., Basics of PET Imagine: Physics, Chemistry, and Regulations. New York: Springer Science + Business Media, Inc., 2005.

Van Eijk, Carel W. E., “Inorganic scintillators in medical imaging.” Physics in Medicine and Biology, vol. 47, no. 8, pp. R85-R106, April 2002.

Van Eijk, Carel W. E., “Inorganic Scintillators in Positron Emission Tomography.” Radiation detectors for medical applications. S. Tavernier, A. Gektin, B. Grinyov, and W. W. Moses eds. Dordrecht: Springer, 2006, 259-274.

Weber, Marvin J., “Inorganic scintillators: today and tomorrow.” Journal of Luminescence, vol. 100, issues 1-4, pp. 35-45, 2002.

Wernick, Miles N., and John N. Aarsvold, eds. Emission Tomography: The Fundamentals of PET and SPECT. Boston: Elsevier Academic Press, 2004.

Woo, Jong-Chun, and Yoon Soo Park, Compound semiconductors 1995: proceedings of the Twenty-Second International Symposium on Compound Semiconductors held in Cheju Island, Korea, 28 Aug. to 2 Sept. 1995. Bristol: Institute of Physics Publishing, 1996. 


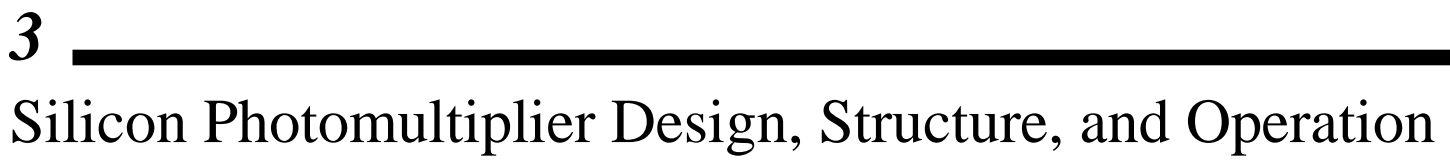

\subsection{Introduction}

Positron emission tomography (PET) relies on the short duration light pulses produced via $511 \mathrm{keV}$ photon interactions within a scintillation crystal. However, the light pulse alone is of little use and must be converted into an electrical signal and amplified. This is achieved by coupling the scintillation crystal(s) to one or more photodetectors. The photodetectors used in PET can be divided into two categories; photomultiplier tubes (PMTs) and solid state semiconductor detectors. While PMTs were the primary photodetector utilized in the development of the first positron cameras and PET systems back in the 1970s (Burnham, 201-205; Ter-Pogossian, 89-98; Muehllehner, 528-537; Derenzo, 544-558; Williams, 619-627) they continue to play a prominent role in a vast majority of commercially available clinical systems available today (Siemens, General Electric, Naviscan; see references). Photomultipliers tubes convert incident scintillation photons into low energy electrons via the photoelectric effect within a thin photosensitive layer known as the photocathode. The low energy electrons are then attracted to the multiplier section of the PMT in which successive positively charged dynodes amplify the number of electrons into a detectible current pulse collected at the anode of the PMT. Photomultiplier tubes are desirable as they offer nearly linear charge amplification (Knoll, 273), high sensitivity with gain on the order of $10^{6}-10^{7}$ (Knoll, 281), high signal to noise, and fast timing ( $\leq 1 \mathrm{ns)} \mathrm{(Del} \mathrm{Guerra,} \mathrm{321).} \mathrm{However,}$ there are limitations which include high bias voltage operation (Biersack, 10-11), low quantum efficiency on the order of $25 \%$ for standard PMTs (Hamamatsu, 143), bulky size, high sensitivity to magnetic fields, and high cost of production.

While solid state semiconductor detectors such as avalanche photodiodes (APDs) attempt to address the drawbacks of PMTs they present their own challenges (discussed below). As a result, considerable attention is being paid to the next generation of solid state semiconductor detector; the silicon photomultiplier (SiPM), which to a large extent, combines the advantages of PMTs and APDs (Roncali, 1360). In recent years there has been myriad investigations into SiPMs as potential photodetectors for PET applications (Li, 590-596; Xie, 1199-1203; Herbert, 389-394; Nassalski, 16201625; Otte, 705-715; Verheyden, 1494-1497). Therefore, in an effort to quantify the novelty and allure of SiPMs for applications in PET, this chapter will explore the fundamental structure and operation of SiPMs as well as outline their principal characteristics such as photon detection efficiency, gain, timing, dynamic range, optical crosstalk, low magnetic field sensitivity, and dark count rate. First however, an insight behind the lineage of the SiPM and an overview of semiconductor diode detector properties is necessary.

\subsection{The Silicon Photomultiplier - An APD Evolution}

While the optimization and refinement of avalanche photodiodes continues to be a current affair, the first experimental evidence for signal amplification via avalanche multiplication was reported by Johnson in 1964 (Johnson, 64-65). Consequently, much of the experimental and theoretical research facilitating the characteristic design and operation of APDs occurred in the 1960s and 1970s and has been documented extensively (Ruegg, 239-251; Biard, 233-238; Lecrosnier, 595-597; Emmons, 3705-3714). Avalanche photodiodes are based on doped $\mathrm{n}$ and p-type semiconductors and the subsequent utilization of advantageous photon detection properties created near the junction between the two. 


\subsection{1 n-type and p-type Semiconductors}

In the case of n-type semiconductors, impurity atoms (donor impurities with concentration $\mathrm{N}_{\mathrm{D}}$ ) are introduced and occupy fixed lattice positions in an otherwise intrinsically undoped semiconductor. However, once the covalent bonds are formed between the impurity atom and the intrinsic lattice atoms there are one or more extra electrons. These extra electrons are loosely bound to the impurity and therefore much more easily excitable up into the conduction band as compared to valance band electrons. As a result, the energy of the extra electrons is such that they may occupy sates (donor levels) within the band gap just below the conduction band (Fig. 3.1a). Consequently, the Fermi energy (Chapter 2) will also reside just below the conduction band amongst the donor levels. Upon thermal excitation of donor level electrons, an excess of conduction band electrons beyond the intrinsic number is produced without the creation of a corresponding hole. Approximately one additional conduction band electron is contributed per donor impurity added. It should however be noted that although there are a significantly greater number of electrons than holes in n-type semiconductors, charge neutrality is maintained as the fixed positively charged ionized donors offset any negative charge contributed by donated electrons.

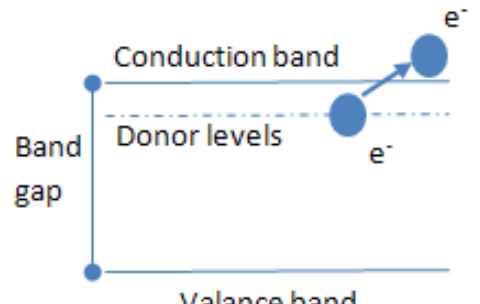

Valance band

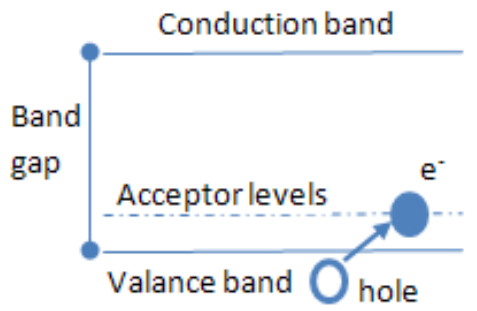

Fig. 3.1a Example band structure of an n-type semiconductor. Donor levels are sufficiently close to the bottom of the conduction band such that the probability of electron excitation is high. This results in excess conduction band electrons.

Fig. 3.1b Example band structure of a p-type semiconductor. Acceptor levels reside just above the valance band such that the probability for valance electron excitation is high. This results in excess valance band holes.

Similar to n-type semiconductors, p-type semiconductors are also characterized by impurities occupying fixed lattice positions within an undoped semiconductor. However, unlike n-type semiconductors the impurities (acceptor impurities with concentration $\mathrm{N}_{\mathrm{A}}$ ) in p-type semiconductors are absent one or more valance electrons compared to the surrounding intrinsic lattice atoms. As a result, the vacancy, which can be characterized as a hole, may capture an electron and form a covalent bond with a surrounding intrinsic lattice atom. Energetically however, since the covalent bond formed is not identical to those of the bulk of the crystal, it is marginally weaker. The captured electrons therefore occupy levels (acceptor levels) within the band gap just above the valance band (Fig. 3.1b). In this case the Fermi energy also resides just above the valance band amongst the acceptor levels.

As a result of the close proximity between the top of the valance band and the acceptor levels, electrons are much more easily thermally excited/captured from intrinsic covalent bonds within the valance band up into the acceptor levels rather than up to the conduction band. This creates an excess of valance band holes beyond the intrinsic number with approximately one additional valance band hole created per acceptor impurity added. Although there are a significantly greater number of holes than electrons in p-type material the fixed negatively charged acceptors will offset any positive charge contributed by excitation induced holes and charge neutrality is maintained. 


\subsection{2 p-n Junction}

Favorable conditions for radiation detection via charge carrier diffusion are created when $\mathrm{n}$ and $\mathrm{p}$-type semiconductors are brought into good thermodynamic contact with each other. This is usually achieved by altering the impurity concentrations on either side of a single intrinsic semiconductor crystal such that distinct $\mathrm{n}$ and $\mathrm{p}$-type semiconductors are formed. The area surrounding the junction between the two semiconductors acts as a conversion element transforming scintillation light into an electrical pulse. Consider first, the junction itself.

Conduction electrons are at a much higher concentration in n-type semiconductors than in p-type semiconductors. Therefore, when the two semiconductors are brought into thermodynamic contact a discontinuity in conduction electron concentration is created. Since conduction electrons are free to migrate there will be a net diffusion into the p-type semiconductor i.e. diffusion along the gradient of high concentration to low concentration such that the flux F of conduction electrons crossing the perpendicular junction plane is given by:

$$
\mathrm{F}=-\mathrm{D}_{\mathrm{n}} \frac{\mathrm{d} \eta_{\mathrm{n}}}{\mathrm{dx}} ; \mathrm{D}_{\mathrm{n}}=\frac{\mathrm{k}_{\mathrm{B}} \mathrm{T} \mu_{\mathrm{n}}}{\mathrm{e}}
$$

with $D_{n}$ equal to the diffusion coefficient for n-type conduction electrons, $d \eta_{n} / d x$ equal to the one dimensional concentration gradient of n-type conduction electrons, $\mathrm{k}_{\mathrm{B}}$ equal to Boltzmann constant, $\mathrm{T}$ equal to temperature, $\mu_{n}$ equal to the conduction electron mobility in the n-type semiconductor (impurity concentration dependent), and e equal to the elementary charge. The negative sign indicates the direction of flow is from high to low concentrations. The flux of conduction electrons through the n-type semiconductor into the p-type semiconductor constitutes a diffusion current $\mathrm{J}_{\mathrm{n}}$ and is given by simply multiplying equation 3.1 by the elementary charge e such that:

$$
J_{\mathrm{n}}=-e D_{\mathrm{n}} \frac{\mathrm{d} \eta_{\mathrm{n}}}{\mathrm{dx}}
$$

Conduction electrons which diffuse into the p-type semiconductor will quickly be captured and replace holes present in the covalent bonding sites within the p-type material. As a result of the conduction electron migration, fixed positively charged ionized donors are left behind in the n-type semiconductor and charge neutrality is no longer maintained. Therefore, in the vicinity of the junction, there is a build up a net positive charge in the n-type semiconductor and net negative charge in the p-type semiconductor.

It should be noted that an analogous view to the diffusion of conduction electrons into p-type semiconductor is the diffusion of holes into the n-type semiconductor. In this case, when the two semiconductors are brought into thermodynamic contact a discontinuity in hole concentration is created. Due to the sharp gradient holes will diffuse from the p-type semiconductor (high concentration) into the $\mathrm{n}$-type semiconductor (low concentration). Both the flux of holes, and subsequent diffusion current $\mathrm{J}_{\mathrm{p}}$, are equal in magnitude yet opposite in direction to the electron flux and diffusion current $\mathrm{J}_{\mathrm{n}}$ respectively, and can therefore be expressed by equations 3.1 and 3.2 by simply changing the sign of the electron parameters such that $-D_{\mathrm{n}}=\mathrm{D}_{\mathrm{p}},-\mathrm{d} \eta_{\mathrm{n}} / \mathrm{dx}=\mathrm{d} \eta_{\mathrm{p}} / \mathrm{dx}$, and $-\mu_{\mathrm{n}}=\mu_{\mathrm{p}}$.

Once in the n-type semiconductor the holes will quickly annihilate with electrons resulting in a disruption of charge neutrality. The positive charge of the fixed ionized donors in the n-type semiconductor will no longer be offset while the holes diffusing into the n-type semiconductor leave behind negatively charged acceptor sites. Again, in the vicinity of the junction there is a build up a net 
positive charge in the n-type semiconductor and net negative charge in the p-type semiconductor (Fig. 3.2).

In the vicinity of the junction between the $\mathrm{n}$ and p-type semiconductors the electronic band structure of each semiconductor bends such that the Fermi energy is continuous across the junction. The band bending represents an increase in electron energy as conduction electrons diffuse into the p-type material. This increase in electron energy corresponds to a decrease in electric potential (conventional defined for a positive charge) and results in an electric potential difference across the junction discussed further below.

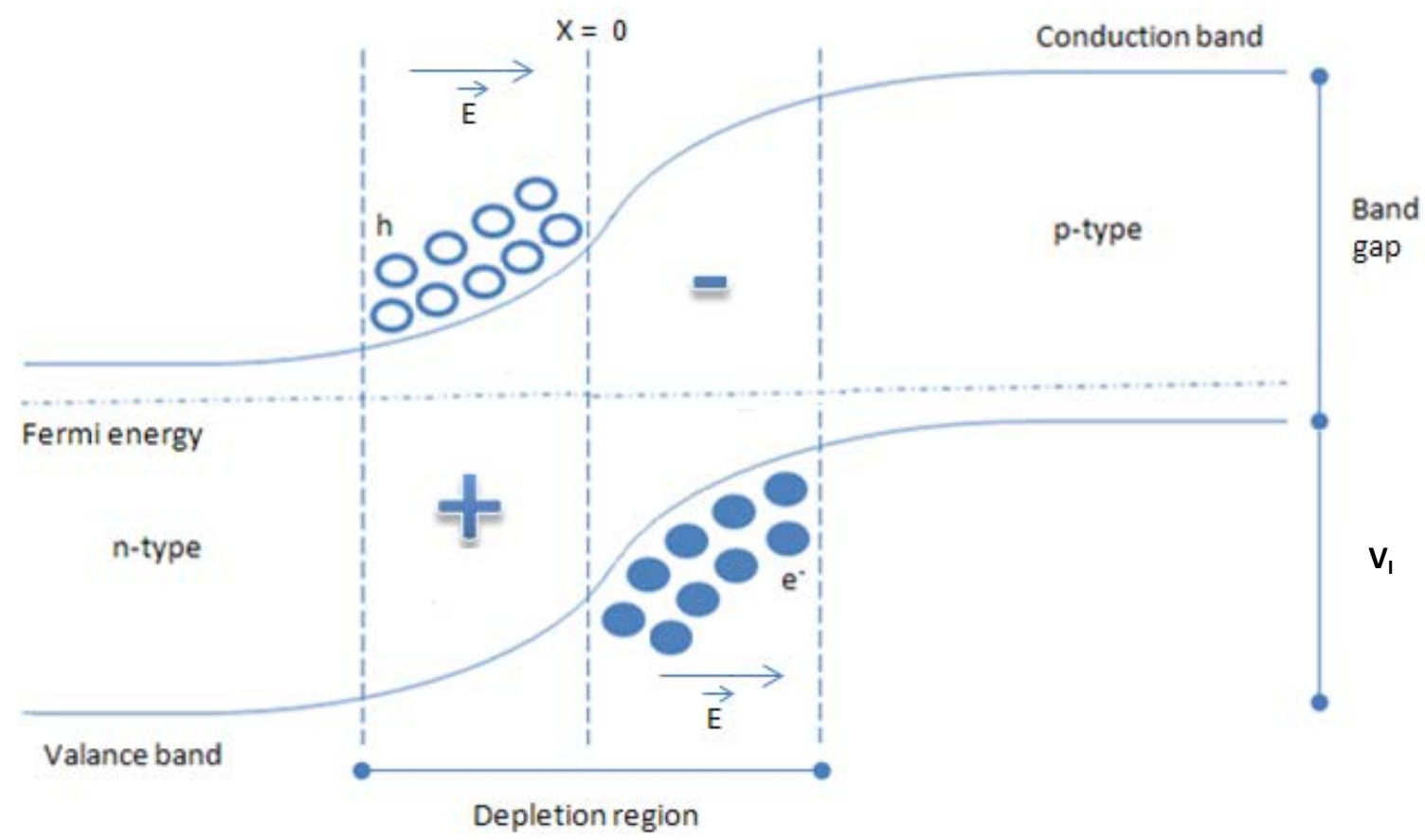

Fig. 3.2 Diagram depicting the band structure and charge carrier diffusion across a p-n junction resulting in a depletion region and subsequent electric field creation.

The region over which charge carrier diffusion has occurred is known as the depletion region. If doping levels are equal in both the $\mathrm{n}$ and $\mathrm{p}$-type semiconductors the deletion region will extend equally into both. As a result of the build up of charge on either side of the junction, an intrinsic electric potential difference (voltage $\mathrm{V}_{\mathrm{I}}$ ) is established and a subsequent electric field $\mathrm{E}$ across the junction is created. It can be shown (Kitai, 75-77) that in terms of the impurity concentrations $\mathrm{N}_{\mathrm{D}}$ and $\mathrm{N}_{\mathrm{A}}$ the intrinsic (contact) voltage $\mathrm{V}_{\mathrm{I}}$ equals:

$$
\mathrm{V}_{\mathrm{I}}=\frac{\mathrm{k}_{\mathrm{B}} \mathrm{T}}{\mathrm{e}} \ln \left(\frac{\mathrm{N}_{\mathrm{A}} \mathrm{N}_{\mathrm{D}}}{\mathrm{n}_{\mathrm{i}}^{2}}\right)
$$

with $n_{i}$ equal to the concentration of conduction electrons in the intrinsic semiconductor comprising the diode.

The electric field inhibits further diffusion of charge carries across the junction and an equilibrium state is achieved with $\mathrm{J}_{\mathrm{n}}=\mathrm{J}_{\mathrm{p}}=0$. The junction can be modeled as a parallel plate capacitor. Consequently, using Poisson's equation and applying the boundary conditions of the electric potential difference spanning the depletion region, one can solve for the separation distance $X_{D}$ between the parallel plates (width of the depletion region) as a function of the impurity concentrations and intrinsic diode voltage (Salivahanan, 94-96) such that: 


$$
\begin{array}{r}
x_{\mathrm{n}}=-\left(\frac{2 \in \mathrm{V}_{\mathrm{I}}}{e \mathrm{~N}_{\mathrm{D}}\left(1+\frac{\mathrm{N}_{\mathrm{D}}}{\mathrm{N}_{\mathrm{A}}}\right)}\right)^{1 / 2} \\
\mathrm{X}_{\mathrm{p}}=\left(\frac{2 \in \mathrm{V}_{\mathrm{I}}}{e \mathrm{~N}_{\mathrm{A}}\left(1+\frac{\mathrm{N}_{\mathrm{A}}}{\mathrm{N}_{\mathrm{D}}}\right)}\right)^{1 / 2} \\
\mathrm{X}_{\mathrm{D}}=\mathrm{X}_{\mathrm{p}}-\mathrm{X}_{\mathrm{n}}=\left(\frac{2 \in \mathrm{V}_{\mathrm{I}}}{\mathrm{e}}\left(\frac{\mathrm{N}_{\mathrm{A}}+\mathrm{N}_{\mathrm{D}}}{\mathrm{N}_{\mathrm{A}} \mathrm{N}_{\mathrm{D}}}\right)\right)^{1 / 2}
\end{array}
$$

with $\mathrm{X}_{\mathrm{N}}$ and $\mathrm{X}_{\mathrm{P}}$ equal to the width of the depletion region extending into the $\mathrm{n}$ and p-type semiconductors respectively, and $\varepsilon$ equal to the absolute permittivity of the depletion region ( $\varepsilon=\varepsilon_{0} \varepsilon_{\mathrm{R}}$ with $\varepsilon_{0}$ equal to the permittivity of free space and $\varepsilon_{\mathrm{R}}$ equal to the relative dielectric constant of the depletion region). Using equation 3.6 one can solve for the junction capacitance $\mathrm{C}_{\mathrm{J}}$ :

$$
\mathrm{C}=\frac{\epsilon \mathrm{A}}{\mathrm{X}_{\mathrm{D}}}=\left(\frac{\epsilon \mathrm{e}}{2 \mathrm{~V}_{\mathrm{I}}\left(\frac{\mathrm{N}_{\mathrm{A}}+\mathrm{N}_{\mathrm{D}}}{\mathrm{B}_{\mathrm{A}} \mathrm{B}_{\mathrm{D}}}\right)}\right)^{1 / 2} \mathrm{~A}=\mathrm{C}_{\mathrm{J}}
$$

where $\mathrm{A}$ is the cross sectional area of the parallel plates (i.e. the surface area of the $\mathrm{n}$ and p-type semiconductors comprising the junction). In order to achieve good energy resolution and mitigate electronic noise levels detectors with as small a junction capacitance are desirable.

The junction acts as a radiation detector in that ionizing radiation (with energy higher than the band gap energy of the diode) incident in or near the junction will create electron-hole pairs with the electrons drifting towards the n-type region and holes drifting towards the p-type region due to the electric field. When the electric field is increased through the application of a reverse bias voltage (discussed below) the generated electrons and holes will gain sufficient kinetic energy such that collisions with the crystal lattice of the diode results in impact ionization and the production of further electrons and holes. A chain reaction in electron hole production ensues and is known as avalanche multiplication. The efficiency of the multiplication process is given by the multiplication factor M:

$$
M=\frac{1}{1-\left(\frac{V_{\mathrm{BB}}}{V_{\mathrm{B}}}\right)^{\mathrm{n}}}
$$

with $\mathrm{V}_{\mathrm{RB}}$ equal to the reverse bias voltage, $\mathrm{V}_{\mathrm{B}}$ equal to the breakdown voltage, and $\mathrm{n}$ equal to a constant ranging from 3 to 6 depending on the semiconductor composition. Breakdown voltage is defined in the following section under Modes of Operation.

The multiplication factor is a statistical average of the number of external electrons which flow for each incident photon and increases as $V_{B}$ increases i.e. as the reverse bias increases gain increases. This flow of charge carriers constitutes a current and is the mechanism by which scintillation photons are converted into a measurable electrical pulse. It should also be noted that when $V_{R B}=V_{B}, M=\infty$ and the diode breaks down. 
There are a number of texts offering a more extensive and in depth insight into solid sate semiconductors as well as p-n junctions and their governing principals although the text by Ashcroft and Mermin is excellent (Ashcroft, 561-614).

\subsubsection{APD Structure and Operation}

Avalanche photodiodes are compact solid state devices based on the p-n junction just described. A schematic diagram of a typical APD is shown in Fig. 3.3 below. The $\mathrm{N}^{+}$and $\mathrm{P}^{+}$notations designate heavily doped $n$ and p-type semiconductors which are used as blocking contacts because of their low minority carrier densities (holes in the n-type and electrons in the p-type).

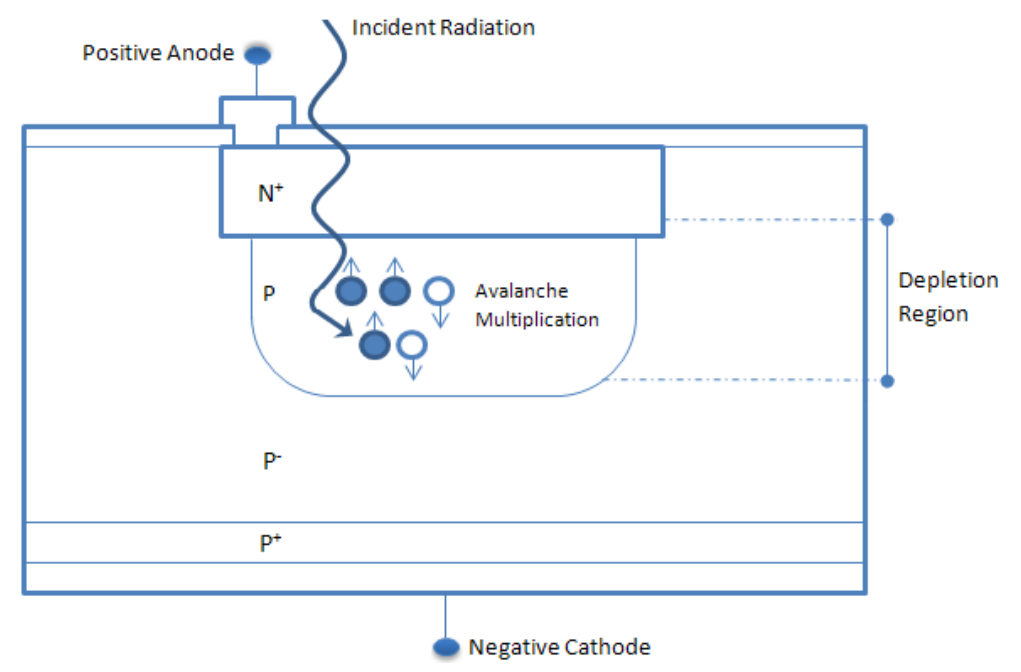

Fig. 3.3 Schematic diagram of a typical APD. Incident radiation is the catalyst for avalanche multiplication within the depletion region.

As described previously, there is an intrinsic potential difference generated across the junction. However, the potential difference is such that the electric field is quite weak and incapable of accelerating generated charge carriers rapidly. The drift speed of electrons and holes depends on the strength of the electric field therefore a weak field results in a high probability for recombination and trapping prior to charge collection. In order to enhance the strength of the electric field the diode is reverse biased. This is achieved by connecting the p-type region to the negative terminal of a power supply and the n-type region to the positive terminal.

Aside from increasing the strength of the electric field, a second consequence of the reverse bias is that the magnitude of holes in the p-type and electrons in the n-type semiconductors that are pulled away from the junction (due to the direction of the electric field) is accentuated. This results in an increase in the width of the depletion region and demonstrates an important parameter of APDs: the width of the active area over which an APD can collect scintillation photons is proportional to the applied reverse bias voltage. Equation 3.6 can be modified to reflect this relationship simply by replacing $V_{I}$ with the sum (reverse) diode voltage $V_{D}$ such that $V_{D}=V_{I}+V_{R B}$.

A third consequence of reverse biasing a diode is the increased resistivity across the junction. This is due to the increased width of the depletion region which represents an increased impedance path to current flow therefore resulting in a higher potential barrier to charge carriers. In general, when compared to non-biased diodes, reverse biased diodes exhibit enhanced junction resistivity and enervated current flow while forward biased diodes exhibit enhanced junction conductivity and increased current flow.

Once a bias voltage is applied, virtually all the applied voltage will appear across the depletion 
region as its resistivity is much higher than the normal undepleted $\mathrm{p}$ and $\mathrm{n}$ material (Knoll, 380). The resistivity of the depletion region is much higher because the only charge remaining in the region are ionized donors and acceptor sites which do not contribute to conductivity because they are fixed and immobile.

Drawbacks to reverse biasing a diode include increased dark (leakage) current and thus increased noise levels. As described above, when reversed biased, the magnitude of majority carriers in the $\mathrm{p}$ and n-type semiconductor (holes in the p-type and electrons in the n-type) pulled away from the junction due to the direction of the enhanced electric field is accentuated. However, concurrently, the enhanced electric field amplifies the intrinsic tendency of minority carriers in each semiconductor (electrons in the p-type and holes in the n-type) to be attracted across the junction. Since the minority carriers in each semiconductor are generated continuously by thermal excitation and are non-lattice bound and free to diffuse, the increased flux of minority carriers attracted across the junction results in the generation of an increased steady state dark current $\mathrm{J}$ across the junction. The net dark current $\mathrm{J}$ is given by sum of the electron dark current and the hole dark current and can be expressed as:

$$
J=J_{0}\left(e^{e V_{D} / k_{B} T}-1\right)
$$

with $\mathrm{J}_{0}$ equal the reverse saturation current. Equation 3.9 clearly demonstrates the dependence of the dark current on applied reverse bias voltage and temperature while also implying the proportionality between dark current and the magnitude of the enhanced electric field as well as width of the depletion region. For a rigorous derivation of equation 3.9 refer to the text by Busch (Busch, 338) or Bube (Bube, 217-218).

\subsubsection{Modes of Operation}

The reverse bias voltage applied to APDs is either just below or slightly above the breakdown voltage of the diode. Breakdown voltage is defined as the minimum voltage at which the resistivity of the p-n junction approaches zero thus allowing self-sustaining avalanche multiplication and constant current flowing through the diode. Diodes biased just below the breakdown voltage operate in proportional mode, while those biased slightly above the breakdown voltage operate in Geiger mode.

Avalanche photodiodes operating in proportional mode are characterized by: 1 . non-selfsustaining multiplication (due to the nonzero resistance of the p-n junction) resulting in an output pulse proportional to the incident scintillation photon flux while still providing gain and 2. zero recovery time as the diode does not need to reset and is still active post detection and as a result can be triggered several times during the measurement gate (Henriksson, 5140). However, one major disadvantage to proportional mode APDs is the significant variance in gain due to the statistical nature and fluctuation of the multiplication factor. This results in an excess noise factor $>2$ (Otte, 2). The excess noise coupled with the fact that $\sim 20$ incident photons (Renker, 48) are required to generate sufficient avalanche multiplications to produce a detectible pulse deem proportional mode APDs unfit for single photon counting applications.

Avalanche photodiodes operating in Geiger mode are better known as single photon avalanche diodes (SPADs). This is due to the fact that since Geiger mode APDs operate above the breakdown voltage a single photon can be the catalyst for self-sustaining avalanche multiplication. This results in a constant flow of current through the p-n junction and a very large output pulse per incident photon. Since the avalanche process is self-sustaining and the current constant any information proportional to the number of scintillation photons deposited in the photodetector (i.e. the energy of the incident radiation prompting the scintillation event) is lost. In order to cease the self-sustaining avalanche multiplication and stem current flow, thereby resetting the diode, quenching circuits are used, and the APD is said to 
operate in limited Geiger mode. There are two types of quenching circuits; simple passive circuits and more elaborate and performance optimizing active circuits (Brown, 4122-4126; Brown, 2383-2389). In order to demonstrate the point and for the sake of brevity, only passive circuits will be discussed.

In the passive circuit approach, the reverse bias voltage is applied to the APD through a load resistor $R_{L}$ of $\geq 100 \mathrm{k} \Omega$. The APD itself has internal resistance of $R_{D}$ (a combination of the junction resistance and the undepleted semiconductor resistance) and junction capacitance $\mathrm{C}_{\mathrm{J}}$ given by modifying equation 3.7 by replacing $V_{I}$ with $V_{D}$. There is also a series resistance $R_{S}$ of the circuit along with a stray capacitance $\mathrm{C}_{\mathrm{S}}$ (Fig. 3.4).

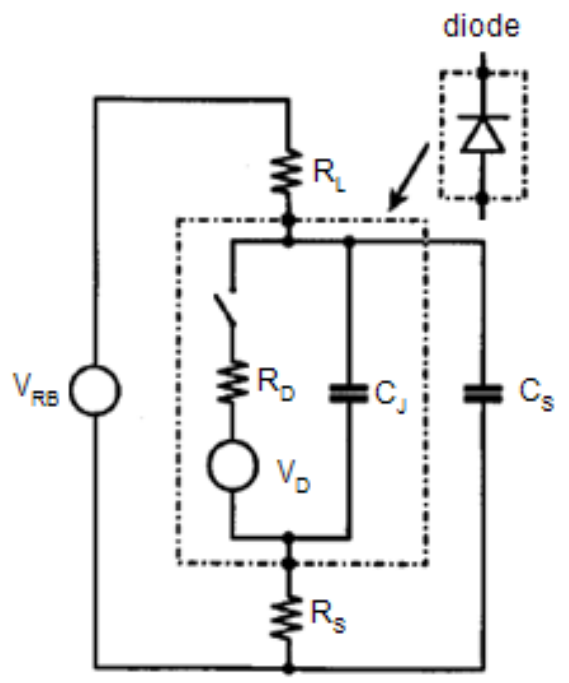

Fig. 3.4 Equivalent circuit for passive quenching of an APD operating in limited Geiger mode (Cova, 1960).

Prior to an incident photon triggering avalanche multiplication both capacitors are charged via the reverse bias voltage $\mathrm{V}_{\mathrm{RB}}$. With the capacitors charged, current flow via the reverse bias voltage equals zero and the voltage across the depletion region of the diode is equal to $V_{D}$. Once an incident photon triggers avalanche multiplication the avalanche current $\mathrm{I}_{\mathrm{A}}$ will discharge the capacitors. As the capacitors discharge, the voltage $V_{D}$ across the depletion region will experience an asymptotic fall towards a steady-sate level. As a result of the voltage drop current via $\mathrm{V}_{\mathrm{RB}}$ will begin to flow through $\mathrm{R}_{\mathrm{L}}$ in order to recharge the capacitors and restore $V_{D}$ across the depletion region. However, since $V_{R B}$ is not connected directly to the diode and instead must flow through $\mathrm{R}_{\mathrm{L}}$ restoring the voltage across the depletion region is not concurrent to the capacitor discharge. Instead, a secondary voltage drop across $\mathrm{R}_{\mathrm{L}}$ will facilitate the asymptotic voltage drop of $V_{D}$ across the depletion region to fall below the breakdown voltage $V_{B}$. This voltage drop below $V_{B}$ results in the asymptotic decay of the avalanche current such that the current is quenched and avalanche multiplication is ceased. As the avalanche current is quenched, the capacitors are recharged via the current through $R_{L}$, and after a characteristic voltage recovery time $T_{R}$, the reverse bias voltage is restored and the diode is reset. The quenching time $\mathrm{T}_{\mathrm{Q}}$ along with the voltage recovery time constitute the dead time for the detector for which subsequent events will not be detected. Quenching time is simply the total parallel capacitance multiplied by the total parallel resistance and since $\mathrm{R}_{\mathrm{L}}>>\mathrm{R}_{\mathrm{D}}$ :

$$
T_{Q}=\left(C_{J}+C_{S}\right) \frac{R_{D} R_{L}}{R_{D}+R_{L}} \simeq\left(C_{J}+C_{S}\right) R_{D}
$$

The voltage recovery time is given by:

$$
T_{R}=\left(C_{j}+C_{S}\right) R_{L}
$$




\subsection{The Silicon Photomultiplier}

Silicon photomultiplier (SiPM) development began in Russia during the 1990s with pursuits centralized around the advent by newly created Metal-Resistor-Semiconductor (MRS) structures and the subsequent evolution of miniature SPADs (Gasanov, 14-17; Bisello, 212-214; Akindinov, 231-234, Saveliev, 223229). The MRS structures allow for large numbers of miniature SPADs to be joined together in parallel on a common silicon substrate and operate under a common load. Each miniature SPAD is commonly referred to as a microcell with typical sizes on the order of 20-30 $\mu^{2}$ (Buzhan, 78). Individual quenching circuitry is integrated into each microcell of which is decoupled from surrounding microcells in order to minimize optical crosstalk. Consequently, each microcell operates as an independent photodetector. Typically there are $10^{3}$ microcells per $\mathrm{mm}^{2}$ however, due to the aforementioned decoupling, the ratio of the active photon detecting area to the overall geometric area of the SiPM, known as the fill factor, is $<1$.

Each microcell is operated in limited Geiger mode with a reverse bias voltage $10-20 \%$ above the breakdown voltage (Otte, 2). Therefore, as discussed in Modes of Operation, the output signal from each microcell is independent of the number of incident photons. The charge $\mathrm{Q}_{\mathrm{MC}}$ accumulated per microcell per event is given by:

$$
\mathrm{Q}_{\mathrm{MC}}=\mathrm{C}_{\mathrm{MC}} \Delta \mathrm{V}=\left(\mathrm{C}_{\mathrm{J}}+\mathrm{C}_{\mathrm{S}}\right)\left(\mathrm{V}_{\mathrm{BB}}-\mathrm{V}_{\mathrm{B}}\right)
$$

with $\mathrm{C}_{\mathrm{MC}}$ equal to the total parallel capacitance of each microcell, and $\Delta \mathrm{V}$ equal to the overvoltage. With the overvoltage being a few volts, and $\mathrm{C}_{\mathrm{MC}}$ typically on the order of $50 \mathrm{fF}, \mathrm{Q}_{\mathrm{MC}}$ is approximately $300 \mathrm{fC}$ resulting in a microcell gain of $\sim 2 \times 10^{6}$ electrons (Dolgoshein, 444).

While each microcell operates as a binary device, the sum signal across all microcells triggered in an event, and readout via a common load resistor, produces an analog output pulse. Therefore, provided that the number of microcells is large compared to the number of incident photons, the amplitude of a SiPM output pulse will be linearly proportional to the intensity of incident photons on the SiPM. Consequently, when coupled to a scintillator, the amplitude of the SiPM output pulse will be linearly proportional to the energy deposited in the scintillation detector, and thus, the energy of the incident ionizing radiation facilitation scintillation photon production.

There are currently a number of companies offering varies designs of SiPMs, with Hamamatsu Photonics, SensL, Photonique SA, Amplification Technologies, Zecotek Photonics, and Advansid being among the leaders (see references). While the design, performance, and operational parameters of SiPMs currently available varies from company to company, the principal defining characteristics of SiPMs in general are outlined as follows.

\subsubsection{Photon Detection Efficiency}

While a single photon incident on an SiPM detector should trigger avalanche multiplication (as $V_{R B}>V_{B}$ ) and a subsequent SiPM output pulse, this is not always the case. This is a result of the photon detection efficiency (PDE) of the detector being less than unity. Photon detection efficiency is defined as the ratio of the number of photons incident on a detector to the number converted into an electrical output pulse and can be expressed as the product of three terms such that:

$$
\operatorname{PDE}[\%]=Q E[\lambda, \mathrm{V}] \times \mathrm{P}_{\mathrm{t}}[\mathrm{T}, \mathrm{V}] \times \mathrm{FF}
$$


with QE equal to the quantum efficiency of the microcells comprising the SiPM, $\mathrm{P}_{\mathrm{t}}$ equal to the triggering probability, and FF equal to the fill factor.

Quantum efficiency, with regards to semiconductor detectors, is defined as the average number of electron-hole pairs created by the absorption of one incident photon in a semiconductor diode (Christensen, 689). For incident photons absorbed in the depletion region the quantum efficiency is unity when the incident photon has energy greater than or equal to the band gap energy of the depletion region and rises above unity if subsequent impact ionization ensues. The quantum efficiency is both wavelength and voltage dependent with the wavelength dependence being two fold.

First, incident photons with short wavelength (blue) will tend to be absorbed just below the surface of the detector prior to reaching the depletion region. Incident photons with long wavelengths (red) will tend to over shoot the depletion region and be absorbed in the non-depleted region of the diode or in the substrate on which the microcell is mounted. In either case any electron hole pair created is quickly lost to recombination resulting in zero percent quantum efficiency. In order to maximize the number of incident photons detected and, thus increase quantum efficiency, the reverse bias voltage may be varied such that the width of the depletion region corresponds to the incident photon wavelength.

Second, the reflective coating on the surface of a SiPM must also be considered as its characteristics are wavelength dependent. In both cases, in order to optimize performance and minimize photons lost, the mutual necessity to match the appropriate photodetector, both in structure and operation, with the appropriate scintillator such that scintillation photons are absorbed in the depletion region is apparent.

Triggering probability is defined as the probability that the electrons or holes created in the depletion region post photon absorption will trigger avalanche multiplication. The triggering probability $P_{t}$ is a combination of the electron and hole triggering probabilities, $P_{e}$ and $P_{h}$ respectively, with $P_{e}>P_{h}$. The triggering probability depends on the ionization coefficients for electrons and holes governing $\mathrm{P}_{\mathrm{e}}$ and $\mathrm{P}_{\mathrm{h}}$ respectively, the shape of the electric field across the depletion region, the location of photon absorption within the depletion region, and increases with increased overvoltage above $V_{B}$ [increased probability for impact ionization] (Oldham, 1056-1060). The triggering probability also exhibits a temperature dependence with the triggering probability increasing as temperature decreases (Rech, 063105-2). Typically, values for the triggering probability $\mathrm{P}_{\mathrm{t}}$ range from 0.5-0.9 (Piemonte, 226).

Of the three terms comprising equation 3.13, the fill factor (defined above) is currently the parameter with the largest effect on PDE (Otte, 4). In order to prevent optical cross talk each microcell is separated by a guard ring, resulting in a few micrometers of dead space surrounding each microcell. As the name suggest, dead space, is inactive and inert to photon detection. Generally, increasing the microcell size increases the fill factor and thus PDE, however, for a fixed SiPM size, increasing the microcell size decreases the dynamic range (disused below) of the detector (Llosá, 877). Currently, SiPMs with fill factors as high as $\sim 80 \%$ have been reported (Hamamatsu).

While PDE varies across SiPM design, mode of operation, and method of measurement, examples of PDE curves as a function of wavelength and temperature can be found in work by Mazillo et al. (Mazillo, 2439-2440). Silicon photomultipliers exhibiting photon detection efficiencies ranging between 5\% (Stewart, 159) to 40\% (Otte [via B. Dolgoshein], 4) have been reported.

\subsubsection{Gain}

The gain of a SiPM is the sum of the charge accumulated by each microcell (equation 3.12) for a given event and depends directly on the magnitude of the reverse bias voltage (overvoltage) and indirectly on 
temperature. In general SiPMs exhibit gain on the order of $10^{6}-10^{7}$ while APDs exhibit a much lower gain on the order of 100-200. Also, in contrast to APDs, which exhibit an exponential dependence between overvoltage and gain, SiPMs exhibit a linear relationship as demonstrated by Petasecca et al. (Petasecca, 1688-1689) for a $1 \mathrm{~mm}^{2} 625$ microcell SiPM from FBK-irst. The indirect relationship between gain and temperature is due to the fact that there is a positive linear relationship between breakdown voltage and temperature which is also demonstrated by Petasecca et al., as well as, Marrocchesi et al. for a $1 \mathrm{~mm}^{2} 3600$ microcell SiPM from Hamamatsu (Marrocchesi, 392-393). If the reverse bias voltage is held constant as temperature is increased the magnitude of the overvoltage decrease therefore decreasing gain. In satellite studies performed at West Virginia University by Majewski et al. average temperature dependent gain drifts of approximately $4.2 \% /{ }^{\circ} \mathrm{C}$ were observed for a 4 x 4 element Hamamatsu MPPC array (S10943-3344MF-050) with 3 x $3 \mathrm{~mm}^{2}$ active area pixels, comprised of 3600 microcells, dry coupled to a $3 \mathrm{~mm}$ thick light spreader window and a $12 \mathrm{x} 12$ element LYSO scintillation array from Proteus, with $1 \times 1 \times 10 \mathrm{~mm}^{3}$ pixels and Toray Lumirror E20 septa, and kept at a constant bias voltage of 74.4V (Fig. 3.5).

(a)

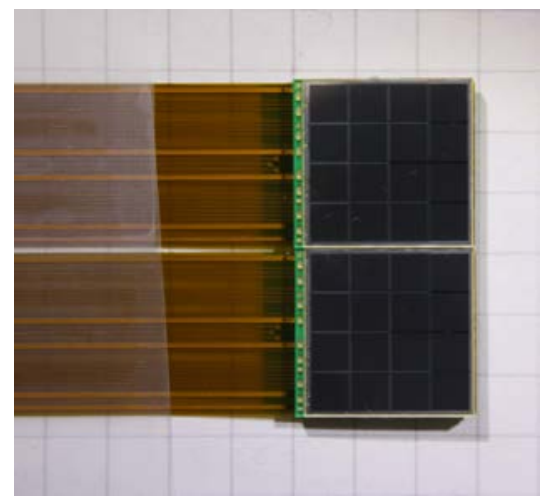

(b)

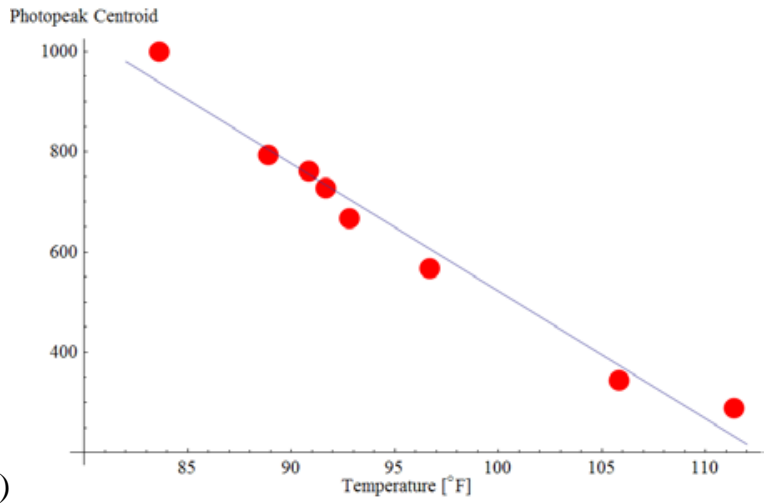

Fig. 3.5 (a) Side by side view of two Hamamatsu MPPCs arrays (S10943-3344MF-050) and (b) gain variations as a function of temperature.

\subsubsection{Timing}

The timing properties of SiPMs are a function of the avalanche breakdown (Geiger discharge) time and the sum recovery time (quenching time plus voltage recovery time; equations 3.10 and 3.11 respectively) averaged across all the microcells triggered in an event. However, first consider a single microcell and a single incident photon.

As the depletion region of each microcell is on the order of 2-4 $\mu \mathrm{m}$ (Renker, 54) the avalanche breakdown time is very fast; typically a few hundred picoseconds. The avalanche breakdown time determines the rise time of the output pulse, with typical rise times observed to be approximately 1 nanosecond. The subsequent decay time of the output pulse is determined by the quenching time of the microcell, with typical decay times around 30 nanoseconds (Buzhan, 34). As equation 3.10 demonstrates, in order for fast timing, microcells with small capacitances are most desirable. It should be noted that since the junction capacitance of each microcell increases with microcell area (equation 3.7) larger microcells exhibit slower timing, and therefore, are less desirable for fast timing applications.

When considering multiple incident photons, the voltage recovery time of each microcell must also be considered when specifying the timing characteristics of SiPMs. In order for a SiPM to be fast not only must the rise and decay time of the output pulse be fast, but so to must be the time required for the bias voltage to be restored and the microcell reset. 
In recent years there has been considerable attention paid to fast timing SiPMs and their applicability to time-of-flight PET (Buzhan, 353-355; Vinke, 188-191, Schaart, N179-N189). When used in such applications (i.e. coupled to a corresponding scintillation detector) the timing characteristics of the measured output pulse of the SiPM also depend on the timing characteristics of the scintillator.

\subsubsection{Dynamic Range}

As discussed earlier, each microcell operates as a binary device with the sum signal of all microcells triggered in an event linearly proportional to the incident photon intensity provided that the number of incident photons is small compared to the number of microcells. The dynamic range of a SiPM is the range of incident photons over which linearity between the SiPM output pulse and the number of incident photons in maintained. Beyond the dynamic range the microcells become saturated and the proportionality breaks down and becomes non-linear. The relationship between the total number of microcells $\left(\mathrm{N}_{\text {microcells }}\right)$, the number of microcells triggered $\left(\mathrm{N}_{\text {triggered }}\right)$, and the number of incident photons $\left(\mathrm{N}_{\mathrm{ph}}\right)$ is given by:

$$
\mathrm{N}_{\text {triggered }}=\mathrm{N}_{\text {microcells }}\left(1-\mathrm{e}^{-\mathrm{PDE} x} \frac{\mathrm{N}_{\mathrm{ph}}}{\mathrm{N}_{\mathrm{microcells}}}\right)
$$

with PDE equal to the photon detection efficiency. Figure 3.6 shows a plot of equation 3.14 for a SiPM with 3600 microcells and PDE values of $10 \%$ - 40\%. From the plot, one can observe that as the number of incident photons increase a non-linear response dominates and saturation occurs. It is also clear from the plot that as the PDE of a SiPM is increased the dynamic range is decreased. As a result, the relationship between dynamic range and detector efficiency is an exercise in compromise.

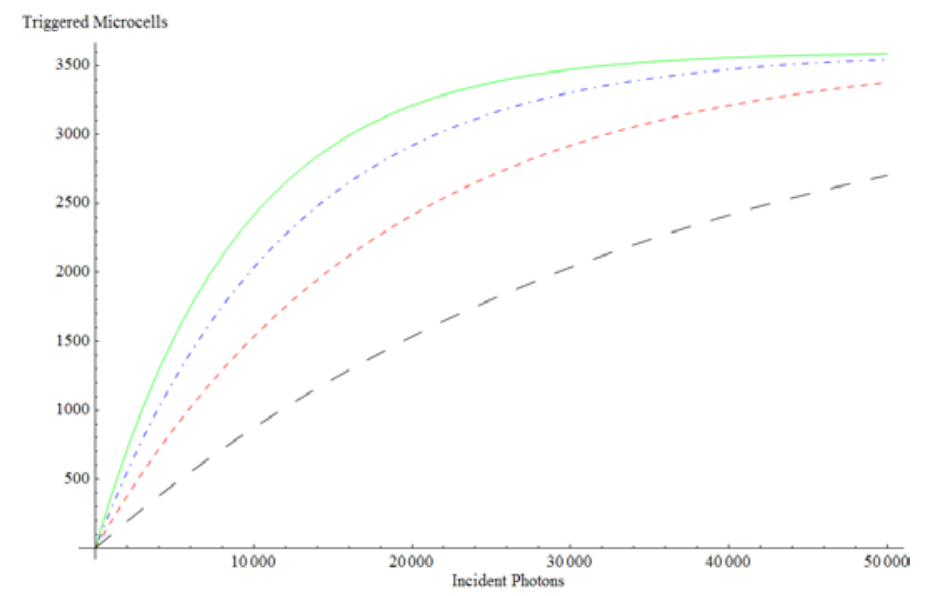

Fig. 3.6 Plot of equation 3.14 for a SiPM with 3600 microcells and multiple different PDE values: $10 \%$, black large dashed; $20 \%$, red dashed; $30 \%$, blue dot dashed; and $40 \%$, green solid. A linear response gives way to a nonlinear response as saturation occurs.

In PET applications the dynamic range of a SiPM is of particular importance. Within the dynamic range the number of triggered microcells is linearly proportional to the energy deposited within the scintillation detector and thus of the incident ionizing radiation. This allows for proper discrimination of events on an energy by energy basis. However, beyond the dynamic range the discrimination of events is impeded, thus degrading the ability to reject Compton-scattered annihilation photons or reject pulse pile-up due to two or more annihilation photons interacting in the same scintillation element at the same time (Roncali, 1365). 


\subsubsection{Optical Crosstalk}

Upon breakdown, avalanching silicon junctions exhibit luminescence as carriers are accelerated across the junction by the strong electric field. Work done by Lucaita et al. (Lucaita, 577-582) builds on prior literature and quotes $2.9 \times 10^{-5}$ photons emitted with energy high than $1.4 \mathrm{eV}$ per carrier crossing the junction. Further work by Akil et al. (Akil, 1022-1028) reviews prior literature examining possible mechanisms for the avalanche spectral response, as well as, presents further interpretations and data on the topic. The photons emitted during avalanche breakdown in one microcell can migrate to surrounding microcells and induce avalanche multiplication. This phenomenon is knows as optical crosstalk and results in the artificial inflation of a SiPM output pulse. Optical crosstalk is a function of the magnitude of the reverse bias voltage as well as the proximity between microcells. However, crosstalk may be minimized by decoupling each microcell from its neighbor by etching trenches between each cell and filling it with optically opaque material (Stewart, 159).

\subsubsection{Low Magnetic Field Sensitivity}

Unlike PMTs, silicon photomultipliers exhibit a low sensitivity to magnetic fields for two reasons. First, as opposed to PMTs, SiPMs are constructed largely of non-magnetic materials. Also, as SiPMs operate at a much lower bias voltage than PMTs, there is no need for magnetically sensitive front end preamplifiers typically required to match the high impedance of a PMTs output pulse with the impedance of associated amplifiers and subsequent electronics. Second, unlike the low energy electrons of PMTs whose trajectory from dynode to dynode can be easily altered by a magnetic field resulting in appreciable gain variations, the large magnitude electric field spanning the narrow depletion region of SiPMs results in high energy electrons exhibiting negligible magnetic field dependence over short distances. Consequently relatively stable gain on the order of $10^{6}-10^{7}$ is observed. In situations where PMTs are used in the presence of a magnetic field, they are often shielded with mu-metal which is held at the PMT photocathode potential.

In recent years, due to their low magnetic field sensitivity, there has been considerable interest in using SiPMs to fuse the functional modality of PET with the structural modality and unsurpassed soft tissue contrast of MRI (McElroy, 106-109; Hong, 882-888, España, 308-316). In this scenario, not only are the SiPMs negligibly affected by the magnetic field, the uniformity of the magnetic MRI field experiences little disturbance due to the non-magnetic structure of SiPMs (as opposed to the magnetic components and shielding of PMTs). This results in negligible degradation of PET detector performance as well as minimization of artifacts created in the MRI image as a result of the PET detector. It should also be noted that eddy currents may be induced within the SiPM due to the large RF field of the MRI, in which case appropriate shield measures of the SiPM are necessary.

In an unpublished manuscript, work at West Virginia University by Majewski et al. investigates the relationship between SiPM performance and magnetic field strength up to 14.1 Tesla (Majewski, 1-2). In this study, custom detectors consisting of $3 \times 3 \mathrm{~mm}^{2}$ SiPMs from Hamamatus (S10362-33-050SMD) arranged in a $4 \times 4$ array with a $5 \mathrm{~mm}$ pitch were designed. The detectors arrays were dry coupled to 2.85 $\mathrm{mm}$ thick light spreader window and a $1 \mathrm{~cm}$ LYSO cube. One detector module was placed perpendicular to the magnetic field and the second transversally to the field. The pulse response from each detector module was measured using an ${ }^{18} \mathrm{~F}$ source with data acquired via a custom designed FPGA based USB system (Proffitt, 2971-2975) and displayed in Kmax. Figure 3.7 below demonstrates the relationship between magnetic field strength and SiPM gain for both the perpendicular (red solid) and transversely (blue dashed) mounted detector modules. While the data is uncorrected for temperature variations a modest $11.8 \%$ decrease in gain for each detector is observed from 0 to 14.1 Tesla. Variations in energy resolution were negligible; ranging from 17.3\% FWHM at 0 Tesla to 17.6\% FWHM at 14.1 Tesla for the transversely mounted detector module. 


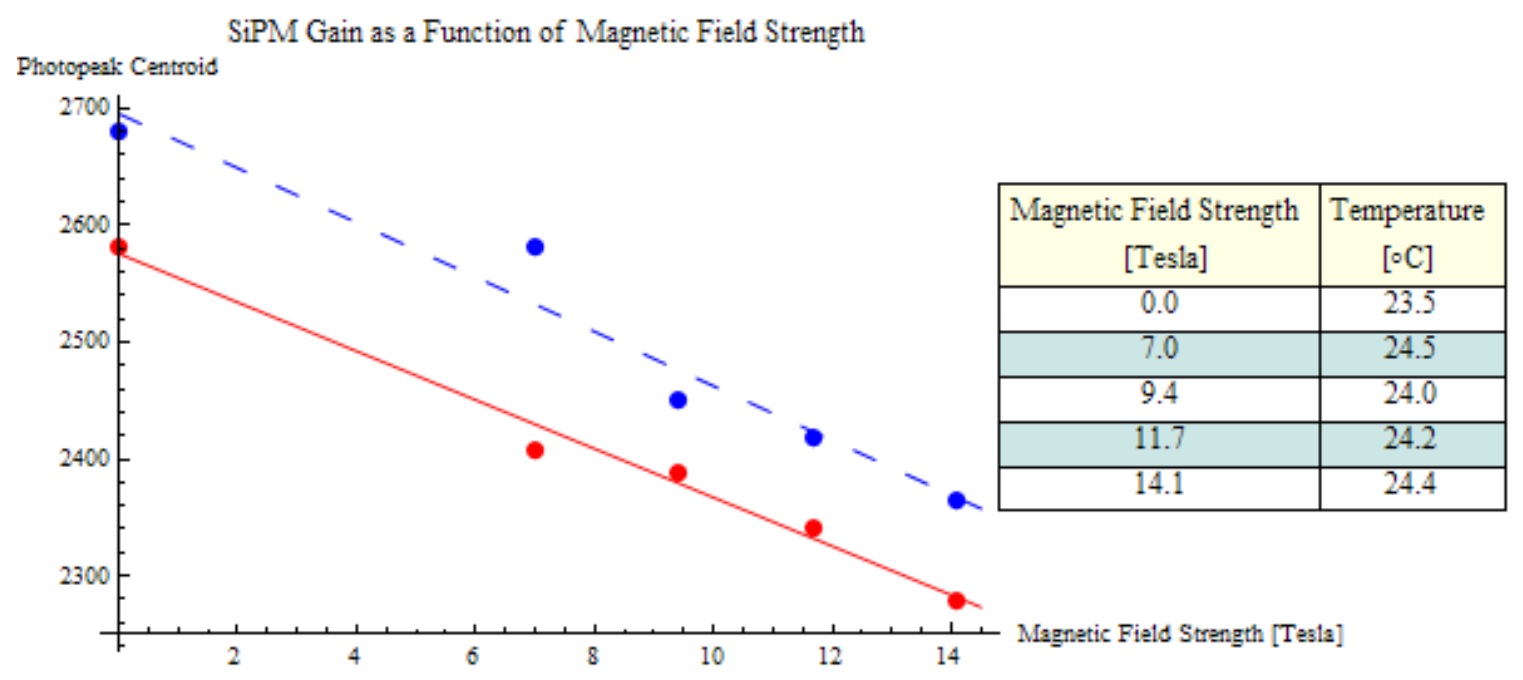

Fig. 3.7 Left: Plot of gain as a function of magnetic field strength for two SiPM based detector modules. One detector module was mounted perpendicular (red solid) to the magnetic field and the other transversely mounted (blue dashed). Right: Temperature of both detectors during the corresponding gain variation measurements.

In a separate study, the scintillation array for the transversely mounted detector module was changed to a $10 \times 10$ element LYSO scintillation array with $1.5 \times 1.5 \times 10 \mathrm{~mm}^{3}$ pixels. All other parameters were unchanged. Data was collected for 3 minutes with figure 3.8 below showing the associated raw images for 0 Tesla (left) and 14.1 Tesla (center). The distortion observed at the bottom left of both images is due gain response non-uniformity for the individual SiPM pixels. The difference between both raw images is shown to the right with no effect on the raw image due to the magnetic field observed.
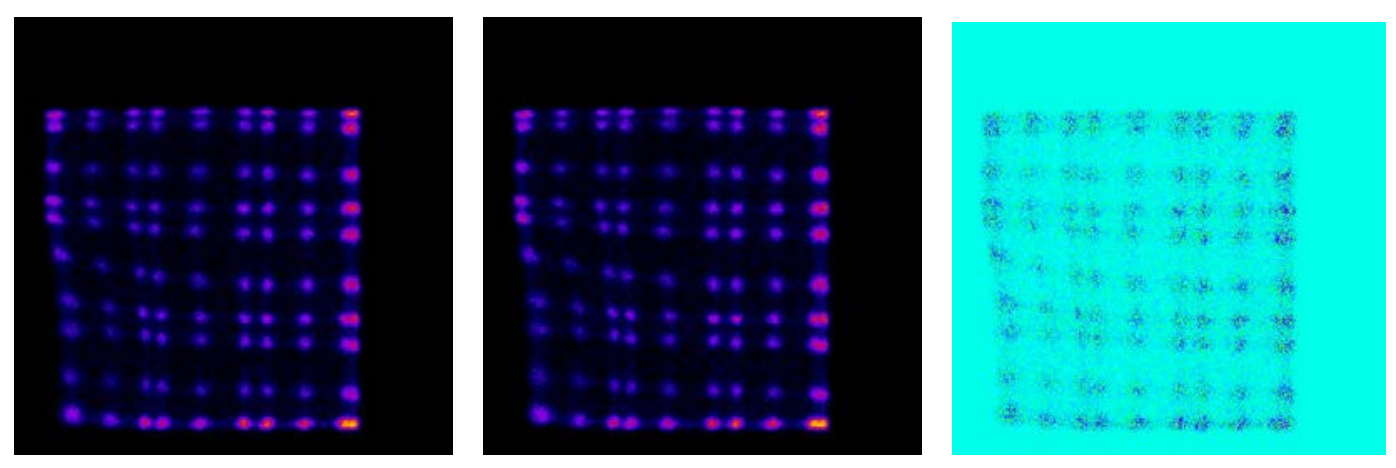

Fig. 3.8 Raw images for the transversely mounted detector module. Left: 0 Tesla, Center: 14.1 Tesla, Right: image difference. No effect on the raw image due to the magnetic field was observed.

\subsubsection{Dark Count Rate}

The most prominent source of noise and one of the main factors limiting SiPM performance is the dark count rate of the SiPM. The dark rate is a result of either thermally generated electron-hole pairs or high electric field assisted generation (tunneling) of free electrons. In either case, the generated free carriers can trigger avalanche breakdown in the detector in the absence of incident photons thus resulting in noise. This noise constitutes a dark or leakage current, as discussed in the section 3.2.3 above, of which is amplified and output as dark pulses. The dark count rate is measured as the average frequency of non- 
photon induced avalanche breakdowns per unit area of the SiPM and depends on the temperature, overvoltage (see equation 3.9) and area of the detector. Typical values for the dark count rate range from $\sim 200 \mathrm{~Hz} / \mathrm{mm}^{2}$ at $100{ }^{\circ} \mathrm{K}$ up to several (typically 1-2) $\mathrm{MHz} / \mathrm{mm}^{2}$ at room temperature (Dolgoshein, 369). Most dark pulses have an amplitude of one photoelectron (Roncali, 1364) however pulses with amplitudes of two or even three photoelectrons are clear evidence for optical crosstalk between microcells (Herbert, 358).

In terms of operational parameters, the magnitude of thermally generated free carriers can be reduced by cooling with a factor two reduction of thermally generated dark pulses for every $8{ }^{\circ} \mathrm{C}$ drop in temperature while the magnitude of field assisted free carries generated can be reduced by operating the SiPM at a lower bias voltage thus resulting in a weaker electric field spanning the depletion region (Renker, 6). The dark count rate can also be reduced at the manufacturing and production level by minimizing the number of generation-recombination centers, impurities, and crystal defects within the SiPM structure (Renker, 52).

\subsection{Overview}

Silicon photomultipliers are avalanche photodiode based semiconductor detectors first developed in the 1990s. In recent years SiPMs have received considerable attention regarding their applicability to PET applications as they, to a large extent, combine the advantages of both PMTs and APDs. While SiPMs present their own challenges and limitations they are undoubtedly the cornerstone of next generation PET scanners especially with regards to time-of-flight (TOF) PET and depth of interaction (DOI) extraction. New breakthroughs such as Philips 64 pixel fully digital SiPM (see references), details of which were presented for the first time at 2010s IEEE Nuclear Science Symposium and Medical Imaging Conference (Degenhardt, conference presentation), are leading the way. Such a device, which integrates both limited Geiger mode micorcells as well as low-voltage CMOS logic (data processing) onto a single silicon substrate, has unparalleled timing performance and low light sensitivity and eliminates the need for noise sensitive analog to digital converters (ADC) (discussed in chapter 4).

This chapter, along with the previous, have covered the two most common and basic components of every PET scanner; the scintillation detector and the associated photodetector. Therefore, the next chapter will examine the basic design and fundamental operation of PET scanners with a special emphasis given to depth of interaction.

\section{References}

Advansid. http://www.advansid.com/.

Akil, Nader, S. E. Kerns, D. V. Kerns Jr., A. Hoffmann, and J. P. Charles, “A Multimechanism Model for Photon Generation by Silicon Junctions in Avalanche Breakdown.” IEEE Transactions on Electron Devices, vol. 46, issue 5, pp. 1022-1028, 1999.

Akindinov, A. V., A. N. Martemianov, P. A. Polozov, V. M. Golovin, and E. A. Grigoriev, "New results on MRS APDs.” Nuclear Instruments and Methods in Physics Research A, vol. 387, issues 1-2, pp. 231-234, 1997.

Amplification Technologies. http://www.amplificationtechnologies.com/.

Ashcroft, Neil W., and N. David Mermin, Solid State Physics. New York: Holt, Rinehart, and Winston, 1976.

Biard, James R., and W. N. Shaunfield Jr., “A Model of the Avalanche Photodiode.” IEEE Transactions on Electrical Devices, vol. 14, issue 5, pp. 233-238, 1967. 
Biersack, Hans-Jürgen, and Leonard M. Freeman, eds. Clinical Nuclear Medicine, New York: Springer, 2007.

Bisello, D., Yu. Gotra, V. Jejer, V. Kushpil, N. Malakhov, A. Paccagnella, Z. Sadygov, I. Stavitsky, and E. Tsyganov, "Silicon avalanche detectors with negative feedback as detectors for high energy physics." Nuclear Instruments and Methods in Physics Research A, vol. 367, issues 1-3, pp. 212-214, 1995.

Brown, Robert G. W., Kevin D. Ridley, and John G. Rarity, "Characterization of silicon avalanche photodiodes for photon correlation measurements. 1: Passive Quenching.” Applied Optics, vol. 25, issue 22, pp. 4122-4126, 1986.

Brown, Robert G.W., Robin Jones, John G. Rarity, and Kevin D. Ridley, "Characterization of silicon avalanche photodiodes for photon correlation measurements. 2: Active Quenching.” Applied Physics, vol. 26, issue 12, pp. 2383-2389, 1987.

Bube, Richard H., Electrons in Solids: An Introductory Survey. $2^{\text {nd }}$ ed. Boston: Academic Press, 1988.

Burnham, C. A., and G. L. Brownell, “A Multi-Crystal Positron Camera.” IEEE Transactions on Nuclear Science, vol. 19, issue 3, pp. 201-205, 1972.

Busch, Georg and Horst Schade, Lectures on Solid State Physics. New York: Pergamon Press, 1976.

Buzhan, P., B. Dolgoshein, A. Ilyin, V. Kantserov, V. Kaplin, A. Karakash, A. Pleshko, E. Popova, S. Smirnov, Yu. Volkov, L. Filatov, S. Klemin, and F. Kayumov, “An Advanced Study of Silicon Photomultiplier.” ICFA Instrumentation Bulletin, vol. 23, pp. 28-41, 2001.

Buzhan, P., B. Dolgoshein, E. Garutti, M. Groll, A. Karakash, V. Kaplin, V. Kantserov, F. Kayumov, S. Klyomin, N. Kondratieva, A. Pleshko, E. Popova, and F. Sefkow, "Timing by silicon photomultiplier: A possible application for TOF measurements.” Nuclear Instruments and Methods in Physics Research A, vol. 567, issue 1, pp. 353355, 2006.

Buzhan, P., B. Dolgoshein, L. Filatov, A. IIyin, V. Kaplin, A. Karakash, S. Klemin, R. Mirzoyan, A. N. Otte, E. Popova, V. Sosnovtsev, and M. Teshima, "Large area silicon photomultipliers: Performance and applications." Nuclear Instruments and Methods in Physics Research A, vol. 567, issue 1, pp. 78-82, 2006.

Christensen, Ove, “Quantum efficiency of the internal photoelectric effect in silicon and germanium.” Journal of Applied Physics, vol. 47, issue 2, pp. 689-695, 1976.

Cova, S., M. Ghioni, A. Lacaita, C. Samori, and F. Zappa, "Avalanche photodiode and quenching circuits for singlephoton detection.” Applied Optics, vol. 35, issue 12, pp. 1956-1976, 1996.

Degenhardt, C., B. Zwaans, T. Frach, and R. de Gruyter, “Arrays of digital Silicon Photomultipiers: Intrinsic performance and Application to Scintillator Readout.” IEEE Nuclear Science Symposium and Medical Imaging Conference, Knoxville, TN, $2^{\text {nd }}$, November, 2010.

Del Guerra, A., N. Belcari, M. G. Bisogni, G. Llosá, S. Marcatili, and S. Moehrs, “Advances in position-sensitive photodetectors for PET applications.” Nuclear Instruments and Methods in Physics Research A, vol. 604, no. 12, pp. 319-322, 2009.

Derenzo, S. E., T. F. Budinger, and J. L. Cahoon, "High Resolution Computed Tomography of Positron Emitters.” IEEE Transactions on Nuclear Science, vol. 24, issue 1, pp. 544-558, 1977.

Dolgoshein, B. "Silicon photomultipliers in particle physics: possibilities and limitations." In Proceedings of the $42^{\text {nd }}$ Workshop on Innovative Detectors for Supercolliders, 28 September - 4 October 2003, World Scientific, Erice, Italy, Singapore, pp. 442-456, 2004. 
Dolgoshein, B., V. Balagura, P. Buzhan, M. Danilov, L. Filatov, E. Garutti, M. Groll, A. Ilyin, V. Kantserov, V. Kaplin, A. Karakash, F. Kayumov, S. Klemin, V. Korbel, H. Meyer, R. Mizuk, V. Morgunov, E. Novikov, P. Pakhlov, E. Popova, V. Rusinov, F. Sefkow, E. Tarkovsky, and I. Tikhomirov, "Status report on silicon photomultiplier development and its applications.” Instruments and Methods in Physics Research A, vol. 563, issue 2, pp. 368-376, 2006.

Emmons, R. B., “Avalanche-Photodiode Frequency Response.” Journal of Applied Physics, vol. 38, issue 9, pp. 3705-3714, 1967.

España S., L. M. Fraile, J. L. Herraiz, J. M. Udías, M. Desco, and J. J. Vaquero, "Performance evaluation of SiPM photodetectors for PET imaging in the presence of magnetic fields.” Nuclear Instruments and Methods in Physics Research A, vol. 613, issue 2, pp. 308-316, 2010.

Gasanov, A.G., V. M. Golovin, Z. Y. Sadygov, and N. Y. Yusipov, "Influences of local nonuniformities in semiconductor substrate on the characteristics of avalanche photodetectors." Letters in the Soviet Journal of Technical Physics. vol. 16, no. 1, pp. 14-17, 1990 (in Russian).

General Electric. http://www.gehealthcare.com/euen/molecular-imaging/index.html.

Hamamatsu Photonics K. K. http://sales.hamamatsu.com/.

Hamamatsu, "Photomultiplier Tubes: Basics and Applications." ${ }^{\text {rd }}$ Edition, [Online] Available: http://sales.hamamatsu.com/assets/applications/ETD/pmt_handbook_complete.pdf [2011, September 7]. Hamamatsu Photonics K. K., Hamamatsu, Japan, 2006.

Henriksson, Markus, "Detection probability for photon-counting avalanche photodiodes applied to a laser radar." Applied Optics, vol. 44, issue 24, pp. 5140-5147, 2005.

Herbert, D. J., N. D’Ascenzo, N. Belcari, A. Del Guerra, F. Morsani, and V. Saveliev, "Study of SiPM as a potential photodetector for scintillator readout.” Instruments and Methods in Physics Research A, vol. 567, issue 1, pp. 356-359, 2006.

Herbert, Deborah. J., V. Saveliev, A. Belcari, N. D’Ascenzo, A. Del Guerra, and Alexie Golovin, “First Results of Scintillation Readout with Silicon Photomultiplier.” IEEE Transactions on Nuclear Science, vol. 53, issue 1, pp. 389-394, 2006.

Hong, Seong Jong, In Chan Song, Mikiko Ito, Sun Il Kwon, Geon Song Lee, Kwang-Souk Sim, Kwang Suk Park, June Tak Rhee, and Jae Sung Lee, "An Investigation Into the Use of Geiger-Mode Solid-State Photomultipliers for Simultaneous PET and MRI Acquisition.” IEEE Transactions on Nuclear Science, vol. 55, issue 3, pp. 882888, 2008.

Johnson, K. M., "Photodiode Signal Enhancement Effect at Avalanche Breakdown Voltage.” Solid-State Circuits Conference. Digest of Technical Papers. 1964 IEEE International, vol. 7, pp. 64-65, 1964.

Kitai, Adrian, Principals of Solar Cells, LEDs and Diodes: The Role of the PN Junction. Chichester: Wiley, 2011.

Knoll, Glenn F., Radiation Detection and Measurement. New York: John Wiley \& Sons, Inc., 1979.

Lacaita, A. L., F. Zappa, S. Bigliardi, and M. Manfredi, “On the Bremsstrahlung Origin of Hot-Carrier-Induced Photons in Silicon Devices.” IEEE Transactions on Electron Devices, vol. 40, issue 3, pp. 577-582, 1993.

Lecrosnier, D., G. Pelous, C. Amouroux, M. Brilman, and G. Ripoche, "Optimization of avalanche silicon photodiodes: A new structure.” 1975 International Electron Devices Meeting, vol. 21, pp. 595-597, 1975. 
Li, Xiaoli, Cate Lockhart, Tom K. Lewellen, and Robert S. Miyaoka, "Study of PET Detector Performance with Varying SiPM Parameters and Readout Schemes.” IEEE Transactions on Nuclear Science, vol. 58, issue 3, pp. 590-596, 2011.

Llosá, G., R. Battiston, N. Belcari, M. Boscardin, G. Collazuol, F. Corsi, G. F. D. Betta, A. Del Guerra, N. Dinu, G. Levi, S. Marcatili, C. Marzocca, C. Piemonte, and A. Pozza, "Novel Silicon Photomultipliers for PET Applications.” IEEE Transactions on Nuclear Science, vol. 55, issue 3, pp. 877-881, 2008.

Majewski, S., J. Proffitt, J. McKisson, R. Raylman, A. Stolin, S. Velan, and A. G. Weisenberger, "Imaging Tests with Silicon Photomultipliers Made from MPPC Arrays in Magnetic Fields up to 14 Tesla.” Unpublished manuscript, West Virginia University, pp. 1-2, 2009.

Marroccheso, P. S., M. G. Bagliesi, K. Batkov, G. Bigongiari, M. Y. Kim, T. Lomtadze, P. Maestro, F. Morsani, and R. Zei, "Active control of the gain of a 3mm x 3mm Silicon Photomultiplier." Nuclear Instruments and Methods in Physics Research A, vol. 602, issue 2, pp. 391-395, 2009.

Mazzillo, M., G. Condorelli, D. Sanfilippo, G. Valvo, B. Carbone, G. Fallica, S. Billotta, M. Belluso, G. Bonanno, L. Cosentino, and P. Finocchiaro, "Silicon photomultiplier technology at STMicroelectronics." IEEE Transactions on Nuclear Science, vol. 56, issue 4, pp. 2434-2442, 2009.

McElroy, David P., Valeri Saveliev, Alla Reznik, and John A. Rowlands, "Evaluation of silicon photomultipliers: A promising new detector for MR compatible PET.” Nuclear Instruments and Methods in Physics Research A, vol. 571, issues 1-2, pp. 106-109, 2007.

Muehllehner, G., M. P. Buchin, and J. H. Dudek, “Performance Parameters for a Positron Imaging Camera.” IEEE Transactions on Nuclear Science, vol. 23, issue 1, pp. 528-537, 1976.

Nassalski, A., M. Moszyński, A. Syntfeld-Każuch, T. Szczęśniak, Ł. Świderski, D. Wolski, T. Batsch, and J. Baszak, "Silicon Photomultiplier as an Alternative for APD in PET/MRI Applications.” 2008 Nuclear Science Symposium Conference Record, pp. 1620-1625, 2008.

Naviscan. http://www.naviscan.com/.

Oldham, W. G., R. R. Samuelson, and P. Antognetti, “Triggering phenomena in avalanche diodes.” IEEE Transactions on Electron Devices, vol. 19, issue 9, pp. 1056-1060, 1972.

Otte, A. N., J. Barral, B. Dolgoshein, J. Hose, S. Klemin, E. Lorenz, R. Mirzoyan, E. Popova, and M. Teshima, “A test of silicon photomultipliers as readout for PET.” Nuclear Instruments and Methods in Physics Research A, vol. 545, issue 3, pp. 705-715, 2005.

Otte, N., "The Silicon Photomultiplier-A new device for High Energy Physics, Astroparticle Physics, Industrial and Medical Applications.” in Proceedings of the IX International Symposium of Detectors for Particle, Astroparticle, and Synchrotron Radiation Experiments, Stanford University, pp. 1-9, 2006.

Petasecca, M., B. Alpat, G. Ambrosi, P. Azzarello, R. Battiston, M. Ionica, A. Papi, G. U. Pignatel, and S. Haino, "Thermal and Electrical Characterization of Silicon Photomultiplier." IEEE Transactions on Nuclear Science, vol. 55, issue 3, pp. 1686-1690, 2008.

Philips, http://www.research.philips.com/initiatives/digitalphotoncounting/index.html.

Photonique SA. http://photonique.ch/.

Piemonte, Claudio, “A new Silicon Photomultiplier structure for blue light detection.” Nuclear Instruments and Methods in Physics Research A, vol. 568, issue 1, pp. 224-232, 2006. 
Proffitt, J., W. Hammond, S. Majewski, V. Popov, R. R. Raylman, A. G. Weisenberger, and R. Wojcik, “A flexible high-rate USB2 data acquisition system for PET and SPECT imaging.” IEEE Nuclear Science Symposium Conference Record, vol. 5, pp. 2971-2975, 2005.

Rech, I., I. Labanco, G. Armellini, A. Gulinatti, M. Ghioni, and S. Cova, "Operation of silicon single photon avalanche diodes at cryogenic temperatures.” Review of Scientific Instruments, vol. 78, issue 6, pp. 063105-1063105-3, 2007.

Renker, D. “Geiger-mode avalanche photodiodes for Cherenkov detectors.” Journal of Instrumentation, vol. 5, P01001, pp. 1-12, 2010.

Renker, D., “Geiger-mode avalanche photodiodes, history, properties, and problems.” Nuclear Instruments and Methods in Physics Research A, vol. 567, issue 1, pp. 48-56, 2006.

Roncali, Emilie, and Simon R. Cherry, "Applications of Silicon Photomultipliers to Positron Emission Tomography.” Annals of Biomedical Engineering, vol. 39, no. 4, pp. 1358-1377, 2011.

Ruegg, Heinz W., “An Optimized Avalanche Photodiode.” IEEE Transactions on Electron Devices, vol. 14, issue 5, pp. 239-251, 1967.

Salivahanan, S., N. Suresh Kumar, and A. Vallavaraj, Electronic Devices and Circuits. $2^{\text {nd }}$ ed. Tata McGraw-Hill, 2008.

Saveliev, V., and V. Golovin, "Silicon avalanche photodiodes on the base of metal-resistor-semiconductor (MRS) structures." Nuclear Instruments and Methods in Physics Research A, vol. 442, issues 1-3, pp. 223-229, 2000.

Schaart, D. R., S. Seifert, R. Vinke, H. T. van Dam, P. Dendooven, H. Löhner, and F. J. Beekman, "LaBr $: C e$ and SiPMs for time-of-flight PET: achieving 100 ps coincidence resolving time.” Physics in Medicine and Biology, vol. 55, issue 7, pp. N179-N189, 2010.

SensL. http://sensl.com/.

Siemens. http://www.usa.siemens.com/.

Stewart, A. G., V. Saveliev, S. J. Bellis, D. J. Herbert, P. J. Hughes, and J. C. Jackson, "Performance of 1-mm² Photomultiplier.” IEEE Journal of Quantum Electronics, vol. 44, issue 2, pp. 157-164, 2008.

Ter-Pogossian, Michel M., Michael E. Phelps, Edward J. Hoffman, and Nizar A. Mullani, “A Positron-Emission Transaxial Tomograph for Nuclear Imaging (PETT).” Radiology, vol. 114, no. 1, pp. 89-98, 1975.

Verheyden, R., S. Korpar, P. Križan, A. Stanovnik, R. Pestotnik, and R. Dolenec, "Performance study of Silicon Photomultipliers as photon detectors for PET.” 2010 Nuclear Science Symposium Conference Record, pp. 1494-1497, 2010.

Vinke, R., H. Löhner, D. R. Schaart, H. T. van Dam, S. Seifert, F. J. Beekman, and P. Dendooven, “Optimizing the timing resolution of SiPM sensors for use in TOF-PET detectors.” Nuclear Instruments and Methods in Physics Research A, vol. 610, issue 1, pp. 188-191, 2009.

Williams, C. W., M. C. Crabtree, and S. G. Burgiss, "Design and Performance Characteristics of a Positron Emission Computed Axial Tomograph- ECATÂ-II.” IEEE Transactions on Nuclear Science, vol. 26, issue 1, pp. 619-627, 1979.

Xie, Qingguo, Chien-Min Kao, Karen Byrum, Gary Drake, Alexandre Vaniachine, Robert G. Wagner, Victor Rykalin, and Chin-Tu Chen, “Characterization of Silicon Photomultipliers for PET Imaging.” 2006 IEEE Nuclear Science Symposium Conference Record, vol. 2, pp. 1199-1203, 2006. 
Zecotek Photonics. http://www.zecotek.com/. 


\section{4 PET: General Concepts}

\subsection{Introduction}

While there is wide variation in PET scanner design, size, configuration, and performance all are based on the coincidence detection of annihilation photon pairs separated by approximately $180^{\circ}$. Annihilation photon detection is actualized via the use of either direct semiconductor based detector modules or indirect scintillation based modules. Direct radiation detection directly converts incident radiation into electrons, and thus, a measurable current pulse while indirect radiation detection first converts incident radiation into light which is subsequently converted into electrons and a measurable current pulse. Examples of direct radiation detectors include silicon strips detectors based on CdTe or CZT. While such detectors often outperform indirect scintillation based detectors, semiconductor detectors can not be reliably manufactured in large quantities at this time due to insufficient crystal growing technology. As a result, a vast majority of PET detector modules rely on indirect radiation detection facilitated by the coupling of scintillation crystal(s) to one or more photodetectors.

Building on the fundamental concepts of the first three chapters, this chapter will focus on how PET utilizes the aforementioned electrical pulse generated by scintillation based detector modules. First, a brief overview of PET scanner design, operation, and data acquisition will be discussed followed by an outline of the general principles of coincidence detection. Next, the concept of depth-of-interaction (DOI) will be explored. Depth-of-interaction is based on the principle that incident annihilation photons usually travel a short distance within the scintillation array of a detector module prior to being detected. Typically, PET scanners do not measure the depth-of-interaction resulting in parallax error and the coupling of sensitivity and spatial resolution of the scanner. For example, increasing the length of the scintillation crystals can increase sensitivity, however, this results in degraded spatial resolution due to increased parallax error. While DOI based PET cameras were first proposed in the 1980s (Karp, 643-655; Rogers, 1061-1090; Rogers 519-522), the advent of solid state photodetectors (particularly SiPMs), has triggered a renewed interest in DOI in recent years. This is due in part to the increasingly compact size of solid state detectors of which enables novel detector geometries for extracting DOI information (reduce parallax error) as well as maximizing the detector module packing fraction (maximized scanner sensitivity). There have been various techniques employed for extracting DOI information with a summary of the most salient approaches discussed in section 4.4 below.

\subsection{PET Scanners and Data Acquisition}

A majority of clinical PET scanners currently in use are based on the block detector originally proposed by Casey et al. (Casey, 460-463). Each block detector is comprised of a block of scintillation crystal of which is segmented into an array of smaller detector elements. Using a saw, the scintillation block is segmented into individual detector elements by making multiple cuts of various depths, with the deepest cuts at the edge of the block. Each cut is filled with an opaque reflective material in order to optically isolate each detector element. Typically, each block detector is optically coupled to four PMTs with each PMT having a proprietary read out channel.

The depth of saw cuts in the block detector are empirically determined to share scintillation light in a linear fashion between the four PMTs as a function of the position of the annihilation photon interaction within the block (Phelps, 22). In order to localize the 2-D X and Y coordinates of annihilation photon interaction within the block detector, and thus in which detector element the interaction occurred, 
a center of gravity calculation is performed on an event by event basis using the four independent PMT signals (Fig . 4.1).

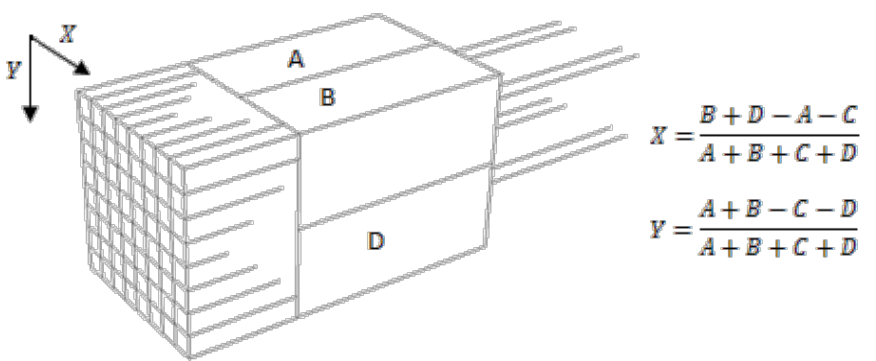

Fig. 4.1 Saw cut block detector with 8 x 8 detector elements mounted to four single channel PMTs. The 2dimensional localization of photon interaction is determined from the four individual PMT signals.

The spatial resolution of each block detector is limited to the size of the saw cut detector elements, with the size of the detector element limited by the light yield of scintillation crystal necessary to perform the center of gravity calculation. Currently, clinical PET scanners are able to achieve a spatial resolution of 4-5 mm FWHM (Rohren, 305-306). A variation on the block detector is the quadrant-sharing detector first proposed by Wong et al. (Wong, 1095-1101). While the quadrant-sharing detector increases spatial resolution accompanying increases in detector dead space and dead time also result.

While there are variations in design and configuration, in general, clinical PET scanners are comprised of multiple block or quadrant sharing detector modules (scintillator + PMTs) arranged in one or more rings or partial rings in either a circular or hexagonal geometry. The total number of rings determines the axial field of view of the scanner and is equal to the number of scintillation detector elements comprising the width of the scanner. For a scanner comprised of $\mathrm{N}$ detector modules, a single module is connected by a coincidence unit to N/2 detector modules, both in plane and axially, on the opposite side of the ring (Saha, 27-28). Therefore, an angle of acceptance, and thus the transverse field of view, is defined for each detector module (Fig. 4.2). The transverse field of view summed across all detector modules comprising the scanner defines the tansaxial field of view.

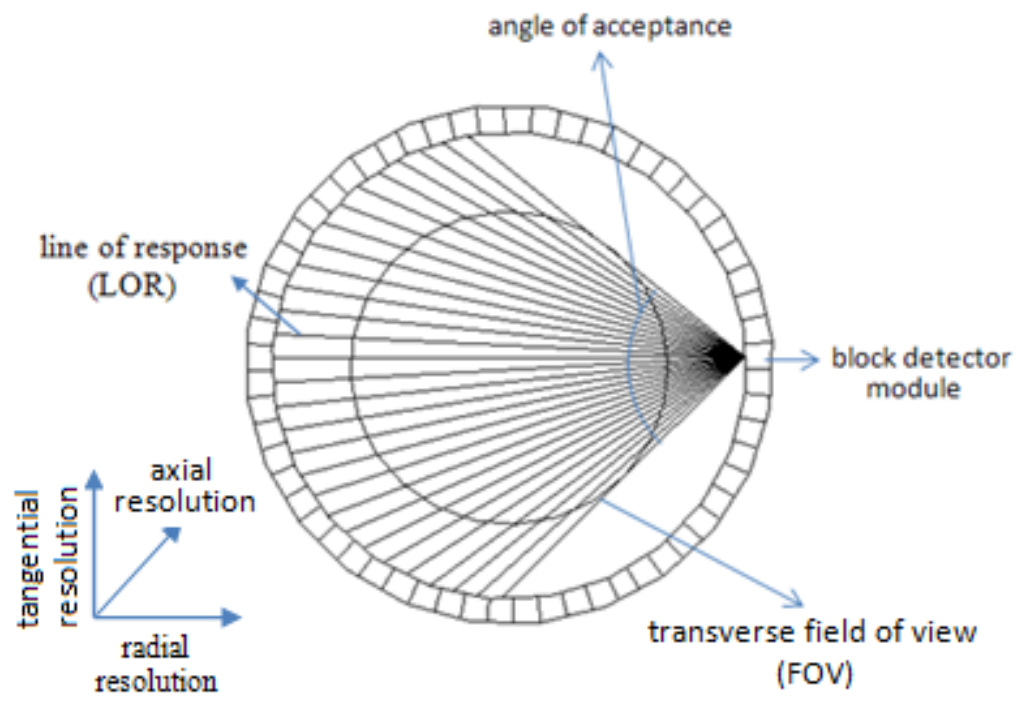

Fig. 4.2 PET ring composed of sixty block detector modules. A single module is in coincidence with N/2 modules on the opposing side of the ring, thus defining an angle of acceptance and the transverse field of view for the single module. The transverse field of view for all modules comprising the ring determines the transaxial field of view of the scanner. 
Once a true coincidence event is detected the localized $\mathrm{X}$ and $\mathrm{Y}$ coordinates of annihilation photon interaction are determined and projected to either the center or the face of the two opposing scintillation detection elements in which the interactions occurred. A line connects both these points and is referred to as the line of response (LOR). With regards to image reconstruction and the creating of a tomographic image, multiple lines of response collected over a range of angles are required.

While clinical whole body PET scanners offer an array of versatility they may not always be practical and or affordable, costing millions of dollars. A practical and more cost effective alternative are dedicated organ and or purpose specific PET scanners. Examples include the breast (Raylamn, 291-295), the brain (Yamamoto, 668-673), the prostate (Huber, 2653-2659), and the imaging of cardiac functionality (Weisenberger, 3705-3708).

Unlike clinical whole body PET scanners, dedicated organ, as well as purpose specific PET scanners, are much smaller in size, requiring significantly less constituent detector components (i.e. photodetectors, scintillators, etc.). This results not only in the reduction in the magnitude and complexity of associated electrics required to readout the detectors, but increased mobility of the imager, as well as significant cost savings. In terms of performance, unlike clinical whole body PET scanners which exhibit poor photon detection efficiency $(<1 \%$ ), a large field of view, and are currently limited to $4-5 \mathrm{~mm}$ FWHM spatial resolution (Rohren, 305-306), dedicated organ and or purpose specific PET imagers offer increased spatial resolution and photon detection efficiency, as well as, a smaller field of view thus reducing image degrading noise from the uptake of background activity from surrounding tissue or bone peripheral to the focal point. The close proximity afforded by dedicated organ and or purpose specific PET imagers, particularly insertable probes, to the anatomical structure being imaged also decreases the amount of injected radioactivity required to produce a statistically sound tomographic image.

The simplest of dedicated PET scanner is the two opposing detector module design. Such a scanner has been implemented at West Virginia University, and is the focus of studies presented in chapter 6. However, in order to outline the fundamentals of coincidence detection the detector modules, as well as the associated electronics comprising the scanner, and subsequent operation there of, will be outlined in the following section. One detector module is a compact depth of interaction (see section 4.4 on DOI) based PET detector while the second module is a simple planer gamma detector. While currently only experimental, the DOI based module is proposed as an endorectal probe placed proximally to the rectal wall/prostate interface and has therefore been designed with both anatomically practical and clinical considerations for prostate imaging. The probe is also proposed to operating in coincidence with one or more externally mounted large area gamma detectors or in tandem with a clinical whole body PET scanner.

\section{$\underline{4.3 \text { Coincidence Detection }}$}

Two detector modules are the minimum number of modules required to enable electronic collimation and coincidence detection. Consider the two modules of the dedicated PET scanner mentioned above. The first module, is compact DOI based PET detector and consists of a 18 x 18 LYSO scintillation array comprised of saw cut $0.7 \mathrm{~mm}$ x $0.7 \mathrm{~mm}$ x $10 \mathrm{~mm}$ pixels (Proteus Inc.). The longitudinal edges of each crystal underwent a special roughing treatment in order to optimize DOI measurement, the necessity of which will be discussed in section 4.4.2. Each pixel was optically isolated in the array via $50 \mu \mathrm{m}$ thick Toray Lumirror E60 septa. The septa is a diffuse white film reflector with a 90\% whiteness index and provides the best compromise between scintillation light yield and thus, crystal identification, as well as DOI resolution. In order to extract DOI information (section 4.4.2.1), both of the polished ends of the scintillation array were optically coupled (Visilox V-788 silicon optical compound from Rhodia Silicones) to a low profile ( 1 mm thick) 4 x 4 element SiPM with $3 \mathrm{~mm}$ x $3 \mathrm{~mm}$ active area pixels (Hamamatsu Photonics K. K.). A $1 \mathrm{~mm}$ thick glass light spreader window was placed between both 
scintillation array/SiPM interfaces. A light spreader window is necessary for scintillation arrays with pixel pitch smaller than the SiPM active element pitch. The window allows scintillation light to spread between neighboring $3 \mathrm{~mm}$ x $3 \mathrm{~mm}$ SiPM pixels in order to localize the scintillation event in 2-D space using the center of gravity method discussed above. The second module, operating in coincidence with the DOI module, consists of either a $1 \mathrm{~cm}$ diameter Hamamatsu R1635 PMT optically coupled to a $1 \mathrm{~cm}^{3}$ LYSO scintillator or a Hamamatsu H8500 PMT optically coupled to a 2 x 2 array of 12 x 12 element LYSO scintillation arrays comprised of $1.0 \mathrm{~mm} \times 1.0 \mathrm{~mm} \times 10.0 \mathrm{~mm}$ pixels depending on the studies being performed.

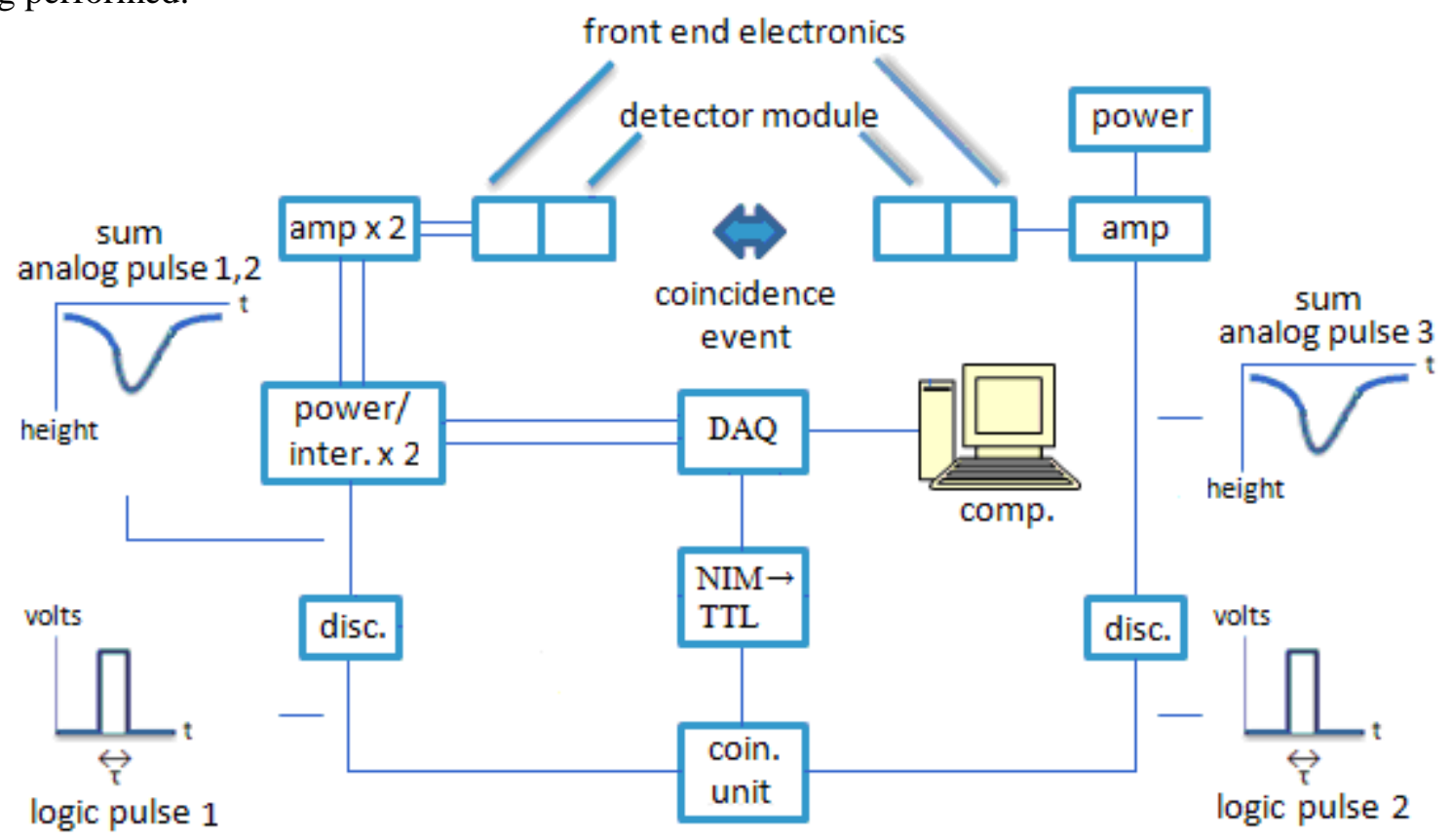

Fig. 4.3 Schematic diagram of basic coincidence detection and associated processing electronics for a DOI based detector module on the left and a gamma detector on the right. (amp=amplifier, inter.=interface, disc.=discriminator, coin.=coincidence, $\mathrm{DAQ}=$ data acquisition, comp.=computer)

In order to illustrate the fundamental operation of a basic coincidence circuit as well as outline the peripheral experimental apparatus architecture, consider the simplest coincidence geometry: the DOI module (left) and gamma detector (right) placed transverse to one another and separated by a distance d (Fig. 4.3). An annihilation event occurring between the two detector modules will produce two approximately back to back photons. So long as both annihilation photons are confined within the volume separating both detector modules prior to incidence, there is a high probability a coincidence event will be recorded. Once an incident photon deposits energy in either detector module, constituent analog pulses proportional to the individual charge build up in each pixel of the photodetector are generated and amplified. Each SiPM of the DOI module is interfaced to a 16 channel amplifier board (Adaptive I/O Technologies, Inc.) via flexible printed circuit (FPC) cables. The amplifier for the second coincidence gamma detector is integrated into the module itself.

With regards to the DOI module, each SiPM amplifier is connected to a custom designed interface box (Adaptive I/O Technologies, Inc.). The box supplies the required SiPM reverse bias voltage as well as sums the constituent analog pulses prior to transmitting the sum pulse from one SiPM (no preference) of the detector module to the discriminator/pulse height analyzer (Philips Scientific Model 715) for analysis. The constituent analog pulses of the gamma detector are summed via the front end electronics and transmitted directly to the discriminator as no interface box was necessary (further explanation forthcoming). 
The discriminator determines if the amplitude of the sum analog pulse, which is proportional to the energy deposited in the scintillation detector of a detector module, and therefore proportional to the energy of the incident radiation, meets or exceeds the specified threshold. As a side note, increasing the discriminator threshold raises the lower limit of the energy acceptance window. Consequently, the allowed energy spectrum is narrowed and results in an increased rejection rate of events corresponding to scattered incident radiation. Therefore, energy resolution of a detector module may be increased by raising the discriminator threshold. However, in doing so, detector module sensitivity is degraded.

If the energy threshold for either detector module is satisfied the discriminator, at time $t_{1}$, produces a NIM logic pulse (digital) of duration $\tau$. The NIM logic pulse is then sent to a coincidence unit (Philips Scientific Model 755) and upon arrival initiates a coincidence time window typically of width $2 \tau$. Depending on the original point of positron annihilation along the distance $d$ separating both detector modules, along with the probabilistic nature of scintillation light yield and scintillation decay time, there will not only be a characteristic difference in arrival time of the second annihilation photon to the second detector module, there will also be a characteristic difference in detection time. Therefore, assuming the sum analog electrical pulse from the second detector module satisfies the energy threshold a second logic pulse of duration $\tau$ will be initiated at a later time $t_{2}$. If the second NIM logic pulse arrives at the coincidence unit within the coincidence time window a valid coincidence event will be detected. The coincidence unit will then produce a single NIM logic pulse, of which is converted to a TTL logic pulse via a gate generator (Philips Scientific Model 794), and transmitted to a data acquisition (DAQ) box. A custom designed FPGA-based USB2 DAQ box (Proffitt, 2971-2975) with a modular extensible architecture with up to 64 channels of simultaneous ADCs per unit, and a sustained trigger rate of over $150 \mathrm{kHz}$ for all channels was used.

Along with the TTL logic pulse, the constituent analog pulses from both SiPMs of the DOI module are also transmitted to the DAQ via flat ribbon cable connected to the custom interface boxes. A separate channel is designated to each individual pixel of each SiPM for a total of thirty-two channels (sixteen per SiPM) readout by the DAQ. Typically the constituent analog pulses from the second detector module, in this case the gamma detector, would be readout by the DAQ as well, however, for the studies being performed, it was not necessary as the gamma detector was used to simply provide electronic collimation.

The rise of the TTL logic pulse from the coincidence unit is detected by the DAQ and a gate signal is generated. The gate signal initiates an integration gate of predetermined duration over which the individual analog pulses from each SiPM are integrated. The temporal delay between the point of sum analog pulse transmittance to the discriminator to the initiation of the integration gate (due to characteristic time required to analyze the pulse(s) in the coincidence circuitry) should be such that the constituent analog pulses transmitted directly to the DAQ from each SiPM fully arrives during the gate duration.

The thirty-two analog pulses readout by the DAQ are integrated by thirty-two ADCs within the DAQ box. Each ADC is composed of a capacitor and a switch. During the integration gate, the analog pulse from each channel charges a capacitor. The sum charge accumulation on each capacitor is a function of the magnitude of each analog pulse, which in turn, is proportional to the magnitude of incident light on each pixel of the SiPMs. Once the integration gate expires the switches are closed and the capacitors discharge, resulting in thirty-two channel specific voltages. As expected, like the charge, each voltage is directly proportional to the magnitude of incident light on each pixel of the SiPMs, with the sum voltage of all thirty-two channels proportional to the magnitude of energy deposited in the scintillation detector. The digitized magnitude of each individual voltage (raw data) is then transmitted from the DAQ to a computer, though a USB-2 connection, for further processing. Java based readout 
software was implemented on the computer with a user interface based on a Kmax scientific software package (Sparrow Corp.).

Utilizing the digitized voltage magnitudes, the software will perform a center of gravity calculation, thus localizing the coordinates of scintillation photon deposition on each SiPM face. The two individual sum magnitudes from each SiPM are also used to calculate the energy and the depth of interaction (section 4.4) of the annihilation photon. Output from these calculations is registered in either list mode or histogram mode and used to calculate the detector modules energy and depth of interaction resolution.

As an aside, when implementing the dual ended readout (section 4.4.21) design just discussed, the number of readout channels can add up quickly thus increasing complexity, as well as, cost. As a result, studies utilizing resistive readouts of which reduced the number of required DAQ readout channels from thirty-two to eight (four per SiPM) are currently being investigated at West Virginia University. Results however, remain preliminary and have yet to be published.

\subsection{Depth of Interaction}

While molecular imaging is becoming an increasingly vital modality, there are performance limitations inherent to the design of clinical PET scanners. One such limitation is the tradeoff between spatial resolution uniformity and sensitivity. For a majority of clinical PET applications, high spatial resolution is necessary in order to delineate small areas of radiotracer uptake, as well as, reduce partial volume effects. The spatial resolution of a scanner is limited to the $x-y$ dimensions of the scintillation crystal(s) employed. Concurrently, high scanner sensitivity is also necessary in order to increase annihilation photon detection efficiency. Increasing a scanner's sensitivity can result in either reduced scan time or the scan duration may remain unchanged and the dose of the injected radiopharmaceutical reduced. Aside from decreasing the discriminator threshold, there are two complementary mechanical methods for maximizing scanner sensitivity. First, the separation distance between detector modules can be minimized. In doing so, the solid angle coverage of the scanner is increased, and therefore, sensitivity is increased. Second, as a result of the penetrating power of $511 \mathrm{keV}$ annihilation photons, scanner sensitivity can be increased through the utilization of "long" scintillation crystals in the z dimension. Currently available state-of-the-art whole body clinical scanners such as the Siemens Biograph TruePoint PET-CT, Philips Gemini TF PET/CT, and GE Discovery PET/CT 690 utilize scintillation crystals of 20, 22, and $25 \mathrm{~mm}$ in length respectively, while older scanners still in use, such as the Siemens Advance NXi, utilize scintillation crystals as long as $30 \mathrm{~mm}$ (factory data; see references).

The compromise between spatial resolution uniformity and sensitivity arises due to depth of interaction effects. As a result of using "long" scintillation crystals, substantial image blur and degradation of spatial resolution, particularity in radial resolution, occurs due to mis-positioning of LORs known as parallax error. Parallax error is a result of annihilation photons incident at oblique angles penetrating more than one scintillation crystal and increases as the radial distance from the scanner's center of field of view increases. Due to multiple crystal penetration, the annihilation photon will typically deposit energy in a secondary or tertiary scintillation crystal at depth $\mathrm{z}$ and not in the initial crystal of incidence (Fig. 4.4).

In the absence of DOI correction, the x-y coordinate of energy deposition over depths within a scintillation crystal is typically projected to either the inner face or the center of the scintillation crystal from which LORs are assigned. Consequently, incorrect LORs corresponding to obliquely incident annihilation photons are generated resulting in image blur and degraded spatial resolution. It should be noted that parallax error may also occur at the center of the field of view if flat panel photodetectors with 
large entrance windows, such as the Hamamatsu H8500, are used, as annihilation photons may be obliquely incident peripheral to the center of the photodetector.
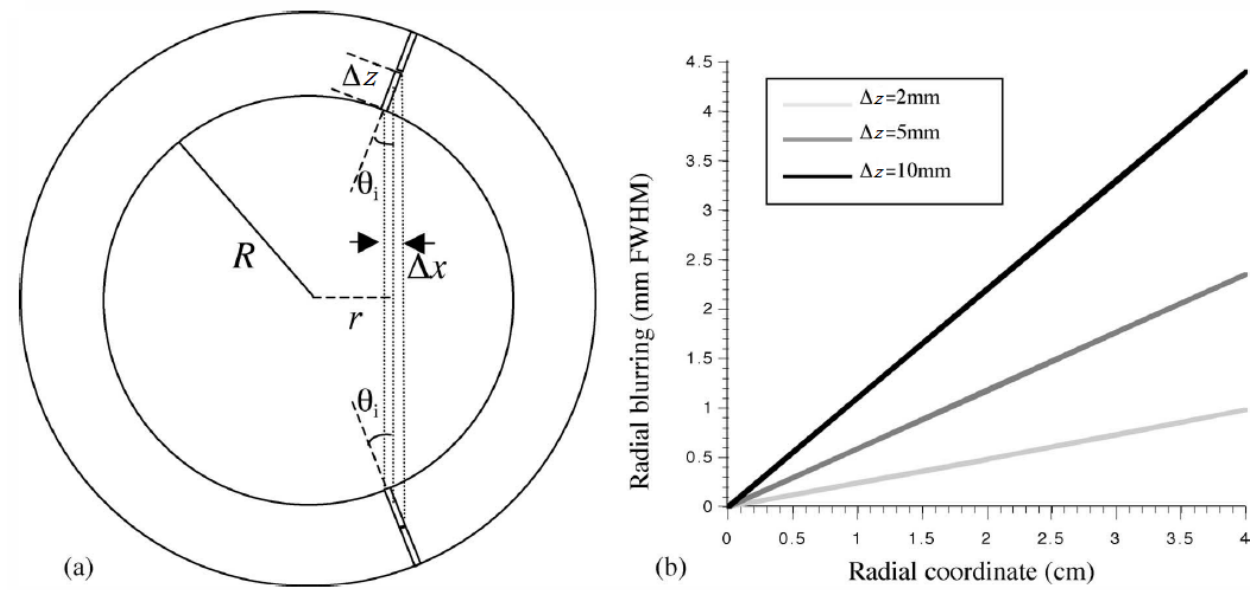

Fig. 4.4 (a) Generic PET ring with inner radius R. Coincidence annihilation photons emitted along radial coordinate $\mathrm{r}$ become increasingly oblique to the initial scintillation crystal of incidence as the distance from the center of the field of view increases. Energy is deposited in a secondary or tertiary scintillation crystal at depth $\mathrm{z}$ and not in the initial crystal of incidence. However, an identical LOR is generated for all three coincident events depicted as the $\mathrm{x}$ y coordinate of energy is projected to either the inner face or center of both scintillation crystals in the absence of DOI information. This results in parallax error and image blur as demonstrated in (b) (Levin, 448).

Parallax error can be appreciably reduced, and nearly isotropic and uniform spatial resolution throughout the entire FOV achieved, via incorporation of DOI information in the reconstruction algorithm. While DOI measurement capability has been incorporated into prototype preclinical dedicated organ PET scanners resulting in an approximately 30\%-60\% increase in spatial resolution near the edge of the scanner's radial field of view (Safavi-Naeini, 3305-3307; Yamaya, 1977-1980), to date, there has yet to be a clinically availably whole body PET scanner offering DOI information extraction and correction. This is due in large part to the increased cost of implementation, as well as, the increased complexity of electronics required to readout such a system. However, within the past decade there has been a steadily expanding interest in the development of DOI encoding dedicated organ and or purpose specific PET probes and scanners. As discussed above, this is due to monetary considerations, the decreased footprint required for the probe/scanner, the increased performance, and, due to the decreased architectural complexity of design, an increased freedom to explore and develop a plethora of novel detector geometries, configurations, and implementations. Such devices range from a mere demonstration of concept to small animal and pre-clinical PET scanners. However, according to a recent DOI review by Ito et al. all future DOI encoding detectors should meet the following criteria: good DOI resolution as to offer an appreciable increase in performance over non-DOI PET, good crystal identification, high stopping power, short dead time, good energy resolution, good time resolution, and low manufacturing cost (Ito, 72).

A review of the current and most salient DOI based detectors, along with the pros and cons of each, will be discussed below. For purposes of clarity, detector designs will be subdivided into either discrete or continuous DOI encoding. Also, a discussion of results obtained in Monte Carlo based simulation studies, such as those performed using GATE simulation toolkits, will be omitted as the results are often idealized and theoretical. 


\subsubsection{Discrete DOI Encoding}

Discrete DOI encoding is typically achieved by optically coupling multiple layers of individual scintillation crystal arrays to a single photodetector. The multi-layer approach can be subdivided into three structural categories: phoswich, light sharing, and relative offset. In general, discrete DOI encoding approaches are appealing as two reasons. First, since a majority of discrete DOI encoding detectors are of a single sided readout architecture there is a significant reduction in photodetector and readout electronics cost as compared to various multi-photodetector continuous DOI encoding designs. Second discrete DOI encoding is typically less complex and logistically easier to implement. However, there are drawbacks. First, while the photodetector cost savings and relatively mollified complexity of discrete DOI encoding facilitate relative ease of DOI extraction, one major drawback is that DOI specificity is equal to the number of layers of the detector with DOI resolution being a function of the axial length of each layer. As a result, in general, discrete DOI encoding results in decreased DOI sensitivity as compared to continuous DOI encoding approaches. Second, in comparison to single layer detectors, multi-layer detectors exhibit degraded light collection efficiency and timing performance due to increased light dispersion throughout the bulk of the scintillation array. Resolution around the periphery of multi-layer detectors is also problematic. However, this is due to a lack of light dispersion near the edges of the detector and is a dilemma for a majority of scintillation based radiation detectors in general.

\subsubsection{Phoswich Detectors}

Phoswich (phosphor sandwich) detectors are based on two or more arrays of optically isolated scintillation crystals layered atop one another and coupled to a single photodetector (Fig. 4.5). Layers are comprised of either distinctly different scintillation crystals or a single type crystal with varied dopant levels in each layer. The light decay time for each layer is different, and thus, the layer in which incident radiation interacts can be distinguished via the distinctly different analog output pulse shapes associated with each layer. There are various methods for pulse shape discrimination including constant fraction discrimination (CFD), constant time discrimination (CTD), charge comparison (CC), rise time discrimination (RTD), and delayed charge integration (DCI) (Chandrikamohan, 398-403; Du, 1757-1771). Examples of phoswich detectors are as follows.
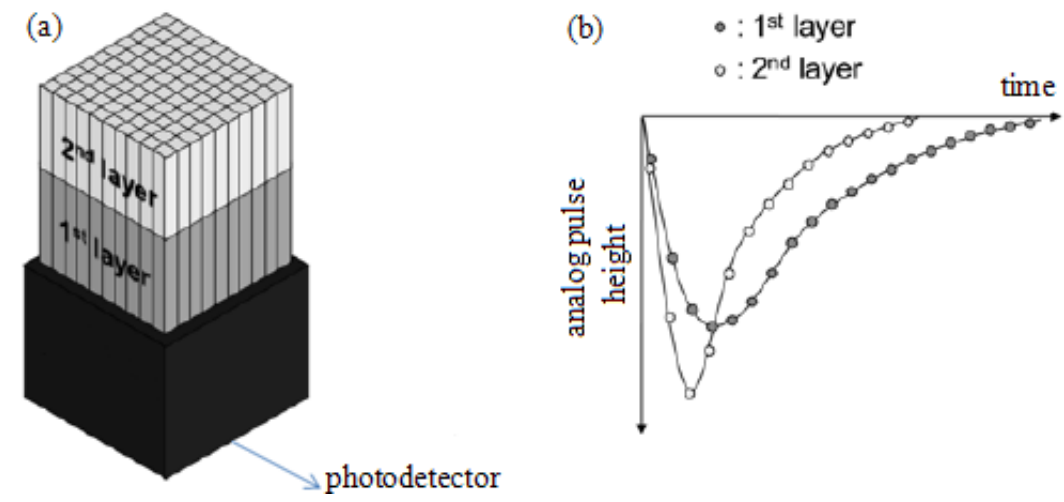

Fig. 4.5 (a) Schematic diagram of a two layer phoswhich detector. (b) Distinctly different analog pulses corresponding to their respective layers (Ito, 73).

Studies by Steun et al. focus on a two layer approach. The first layer consisted of LuYAP70 or LuYAP80 with the second layer consisted of LSO. All crystals were polished and measured $2 \times 2 \times 10$ $\mathrm{mm}^{3}$ and were coupled to a single PMT. Upon irradiation the reliability to distinguish LSO pluses from LuYAP70 pulses was $99.2 \%$ and $97.7 \%$ for LuYAP80 pulses. Energy resolution for the detector was not quoted (Steun, 344-347). Studies by Jung et al. also focus on a LSO/LuYAP dual layer phoswich 
detector of the same dimensions and coupled to a PMT. Upon irradiation the DOI misclassification error for LSO and LuYAP pulses was $0.2 \%$ and $2.4 \%$ respectively. Energy resolution for the LSO layer was 28.1\% FWHM and 21.1\% FWHM for the LuYAP layer (Jung, 669-675). While both studies exploit the slow component of light decay in LuYAP scintillators and provide encouraging results, they are only for a single layered pixel and whether such results will be maintained in a larger PET system is unknown.

One potential problem with the LSO/LuYAP combination is that the LuYAP emission spectrum overlaps the excitation spectrum of LSO, thus altering the emission of LSO such that the photo luminescence may have a decay time quite different from the decay time from gamma excitation (Eriksson, S288-S292). As a result of this potential problem, studies by Eriksson et al. focus on various other phoswich combinations with the first layer consisting of either LSO, GSO, or YSO, and the second layer consisting of either $\mathrm{LaBr}_{3}$ or $\mathrm{LaCl}_{3}$.

Recent studies by Vaquero et al. focus less on layer optimization and performance and more on the performance of the photodetector used to readout the crystal layers. As demonstrated above, phoswich detectors readout by PMTs produces excellent layer identification and respectable energy resolution. However, the performance of a SiPM based phoswich detector has yet to be well established. In an effort to quantify the performance of a SiPM based phoswich detector, Vaquero utilizes a 3 x 4 array of GSO scintillation crystals for the first layer and LYSO for the second layer. A reflector is placed between the layers to facilitate uniform light distribution. The two layers were coupled to either a $2 \times 2$ element Hamamatsu SiPM (6 mm x 6mm) or a 4 x 4 element SensL SiPM (15.8 mm x 15.3mm). Initial results indicate that the timing performance of SiPMs preserves the shape of the output analog pulse corresponding to the light decay in each layer. Therefore, layer misidentification is expected to be minimal and the uncertainty of SiPMs as a PMT replacement in phoswich detectors assuaged (Vaquero, 3311-3313).

While phoswich detector architecture and performance continues to be improved, one example of a prototype pre-clinical scanner currently utilizing phoswich based detector modules is the jPET-D4. The jPET-D4, a high performance dedicated PET brain scanner, is currently under development at the National Institute of Radiological Sciences (NIRS) and it composed of 5 rings of 24 block detectors each. Each block detector is comprised of 4 layers of 16 × 16 arrays of $2.9 \times 2.9 \times 7.5 \mathrm{~mm}^{3}$ GSO scintillation crystals coupled to a PSPMT. Each layer is doped with a different amount of cerium. Utilizing a DOI compression method, initial phantom studies indicate that the jPET-D4 scanner can achieve uniform spatial resolution of 2-3 mm across the entire field of view (Yamaya, 1977-1980; Kitamura, 231-234)

A new design scheme for a phoswich based PET detector module has been proposed by Wei et al. Unlike convention phoswich detector modules, the varied scintillation crystals are alternated in the $x-y$ plane and not in the $\mathrm{z}$ plane. This results in increased event localization in the transverse plane, especially for high dimension crystal arrays. However no DOI information is extracted (Wei, 2874-2876).

\subsubsection{Light Sharing Detectors}

Similar to phoswich detectors, the light sharing approach also relies on layers of multiple scintillation crystal arrays coupled to a single photodetector. There are currently two architectural variants to the light sharing approach; the disparate layer based design, and the intra-layer light spreader window design.

First, the disparate layer based design as illustrated by Tsuda et al. (Fig. 4.6). The detector is comprised of 4 layers of identical GSO scintillation crystals stacked atop one another and coupled to a single PSPMT. Each layer is a $16 \times 16$ array of $1.42 \times 1.42 \times 4.5 \mathrm{~mm}^{3}$ etched scintillation crystals, however, a novel, layer specific arrangement of reflective material and air gaps between individual crystals distinguishes one layer from another. As a result of the air gaps, scintillation photons are shared 
between surrounding crystals and, when coupled with the unique reflector arrangement of each layer, the light distribution centroid for each crystal is shifted such that it is possible for each crystal of interaction to be expressed on a single 2-D position histogram without overlapping. Thus, DOI of an event is simply determined its resultant 2-D histogram position. However, the DOI misidentification rate is a more subjective matter as the ratio between histogram peaks and valleys is the metric used to quantify DOI accuracy. In general, as the number of layers is increased, the peak-to-valley ratio diverges towards unity and DOI accuracy decreases. This is a consequence of increased scatter, attenuation, and light dispersion within the crystals and the resulting mis-positioning of events in the flood histogram.
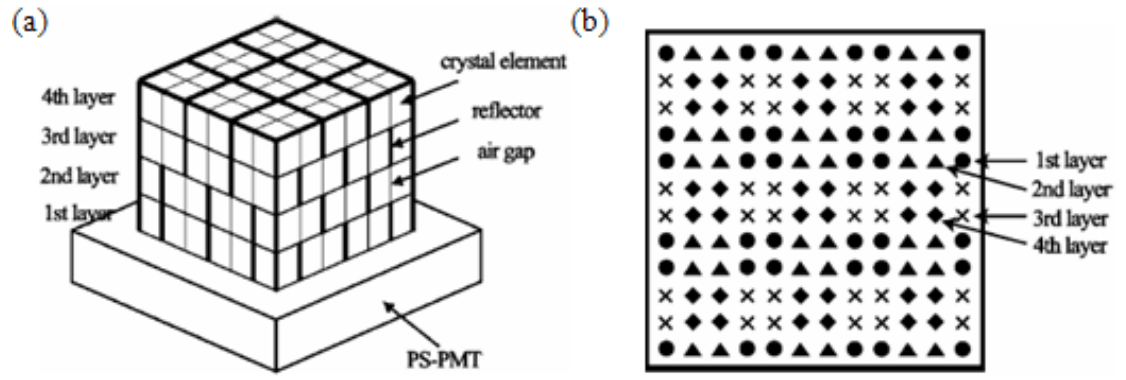

Fig. 4.6 (a) Schematic diagram of a four layer light sharing DOI detector. The disparate air gap and reflector arraignment of each layer shifts the centroid of light distribution of each crystal such that each crystal of interaction is expressed on a single 2-D histogram (b) without overlapping.

Studies were performed using both a collimated fan beam to irradiate reach layer separately and a broad beam to uniformly irradiate the entire detector. On a layer by layer basis, initial histogram images for the collimated beam show all pixels clearly separated except around the edges. Encouraging initial results utilizing the broad beam are presented for a central portion of the detector and demonstrated the ability to resolve individual crystals across all four layers on a single 2-D histogram. Energy resolution decreased as layer number increased (due to increased attenuation of higher layer scintillation photons) and, while initially encouraging, was only measured for a single centrally located crystal stack. For layers one through four energy resolution equaled $11.2 \%, 11.5 \%, 12.6 \%$ and $13.7 \%$ respectively (Tsuda, 25372542).

Follow up studies expanded the detector to a hybrid phoswich eight layer light sharing detector. However both pixel separation and energy resolution for all layers was worse (Inadama, 2766-2769). The most recent studies have investigated the applicability of SiPMs to the light sharing design. Adopting the reflector arrangement proposed by Tsuda above and utilizing a 4 x 4 element SiPM coupled to a four layer scintillation array with each layer consisting of $6 \times 6$ array of $1.46 \times 1.46 \times 4.5 \mathrm{~mm}^{3}$ LYSO scintillation crystals initial results proved similar to prior results when employing a PSPMT (Nishikido, 3923-3925).

While results for the disparate layer based design are promising, two major drawbacks to such an approach are the extremely high mechanical precision required to fabricate and align such intricate multilayer arrays, as well as the increased financial cost to do so. Consequently, in an attempt to mitigate both the cost and design and fabrication complexities inherent to the disparate approach, as well as avoid the complex algorithms utilized for pulse shape discrimination, an alternate light sharing design is simplistically based on placing a transparent light spreader window between two identical scintillation arrays stacked atop one another and coupled to a single photodetector. In doing so, the depth encoding is solely based on the width of the light distribution on the face of the photodetector instead of spatial encoding indicative of the disparate layer approach. However, similar to the disparate approach the centroid of light distribution for each scintillation crystal in the top layer is shifted allowing for both the 
$\mathrm{x}-\mathrm{y}$ position and the layer of interaction to be expressed on a single 2-D flood histogram without overlapping. Studies by Düppenbecker et al. utilize two identical 32 x 32 element scintillation arrays composed of polished LYSO crystals with a $1 \mathrm{~mm}$ pitch and optically separated by a specular reflector. The arrays are stacked with a light spreader window placed in between and coupled to a digital SiPM. While initial results demonstrate the ability to distinguish the layers based on the width of the light distribution while achieving an energy resolution of 13\% FWHM for both layers, a detailed analysis was only performed on a single crystal stack (Düppenbecker, 2252-2253).

Other more extensive studies by Kang et al. explore the use of 2 layers of 4 x 4 element LYSO scintillation arrays comprised of $3 \times 3 \times 10 \mathrm{~mm}^{3}$ optically isolated crystals separated by a light spreader window of either 3 or $5 \mathrm{~mm}$ in thickness and coupled to a SiPM (Fig. 4.7). For both windows all crystals were clearly resolved with the $3 \mathrm{~mm}$ window providing the best average energy resolution of $15.1 \%$ FWHM and 14.6\% FWHM for the top and bottom layers respectively (Kang, 2859-2864).

(a)

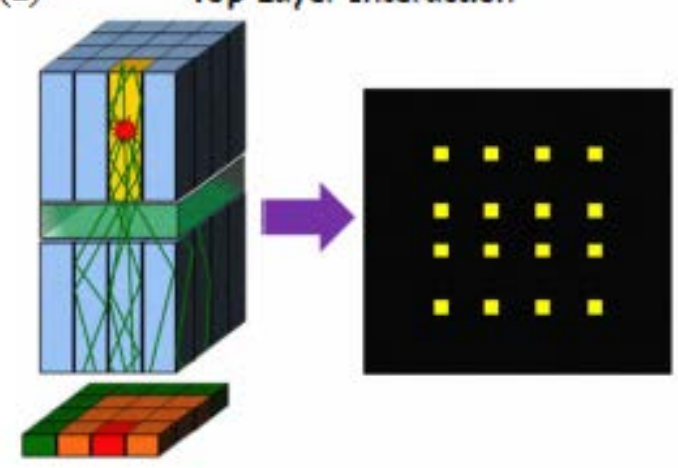

(b)

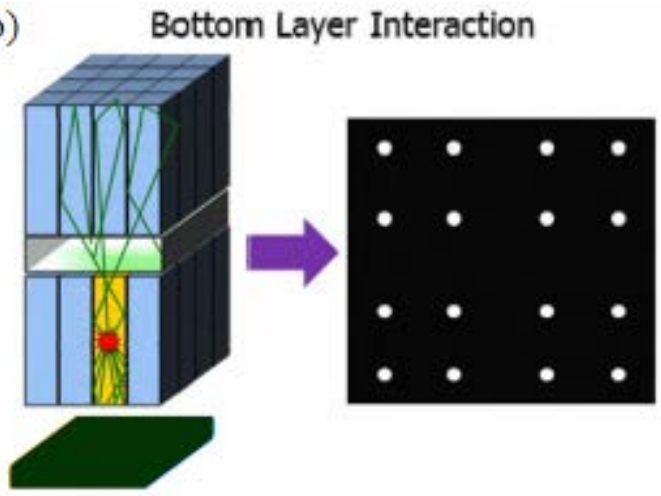

Fig. 4.7 Schematic diagrams of the intra-layer light spreader window approach along with corresponding flood histograms for uniform irradiation of both the top (a) and bottom (b) layer. The centroid of light distribution for each scintillation crystals in the top layer is shifted allowing for both the $x-y$ position and the layer of interaction (a function of the width of the light distribution) to be expressed on a single 2-D flood histogram without overlapping.

\subsubsection{Relative Offset Detectors}

Similar to both the phoswich and light sharing approaches above the relative offset design is a multi-layer approach as well. However, instead of stacking the scintillation arrays precisely one on top of the other each layer is offset by one half a crystal pitch in both the $\mathrm{x}$ and $\mathrm{y}$ direction. As with the light sharing approaches, this method shifts the centroid of light distribution for each crystal in the top layer(s) allowing for all crystals in all layers to be separately expressed on a single 2-D flood histogram. However, along with the aforementioned drawbacks of multi-layer detectors, an extremely accurate alignment of each layer is required in order to mitigate the overlap of crystal centroids in the flood image. Examples of the relative offset approach include studies by both Lie et al. and Belcari et al. The detector constructed by Liu utilized both an upper $6 \times 6$ array and lower $7 \times 7$ array of $1.8 \times 1.8 \times 10 \mathrm{~mm}^{3}$ optically isolated LSO scintillation crystals. The upper layer was off set by one half crystal pitch while the lower layer was coupled to a PSPMT (Fig. 4.8a). When uniformly irradiated each crystal element was clearly separated on a single 2-D flood histogram with energy resolution in the lower array averaging 20.7\% FWHM and 21.9\% FWHM in the upper array. In further studies the spatial resolution in coincidence was measured for a pair of two layer offset detectors at different angles of incident radiation. At $0^{\circ}$ the spatial resolution was $1.4 \mathrm{~mm}$ FWHM and at $30^{\circ} 3.4 \mathrm{~mm}$ FWHM as compared to $1.5 \mathrm{~mm}$ FWHM and $4.5 \mathrm{~mm}$ FWHM respectively for non-DOI encoding detectors thus proving the incorporation of DOI information markedly deduces the impact of parallax error and increases spatial resolution (Liu, 182-190). 
Studies by Belcari et al. utilize a PSMPT along with two $5 \mathrm{x} 5$ arrays of YAP scintillation crystals. The crystal dimensions were $2 \times 2 \mathrm{~mm}^{2}$ with the top layer measuring $10 \mathrm{~mm}$ thick and the bottom layer $20 \mathrm{~mm}$ thick. Upon uniform irradiation all 50 crystal elements were correctly identified on the flood histogram thus adding further validity to the results obtained by Liu (Belcari, 246-247).

Recent studies by Ito et al. have expanded the relative offset approach to four layers. Utilizing $1.5 \times 1.5 \times 7 \mathrm{~mm}^{3}$ LYSO scintillation crystals four staggered arrays were coupled to a single PMT. Upon uniform irradiation all crystals in the center of the detector were clearly identified while those near the edge were less well separated. Energy resolution decreased from 13.3\% FWHM in the first layer to 25\% FWHM in the fourth layer as a result of the offset structure causing increased scintillation photon scattering and decreased light collection efficiency (Ito, 976-981). However, overall, initial results are encouraging.
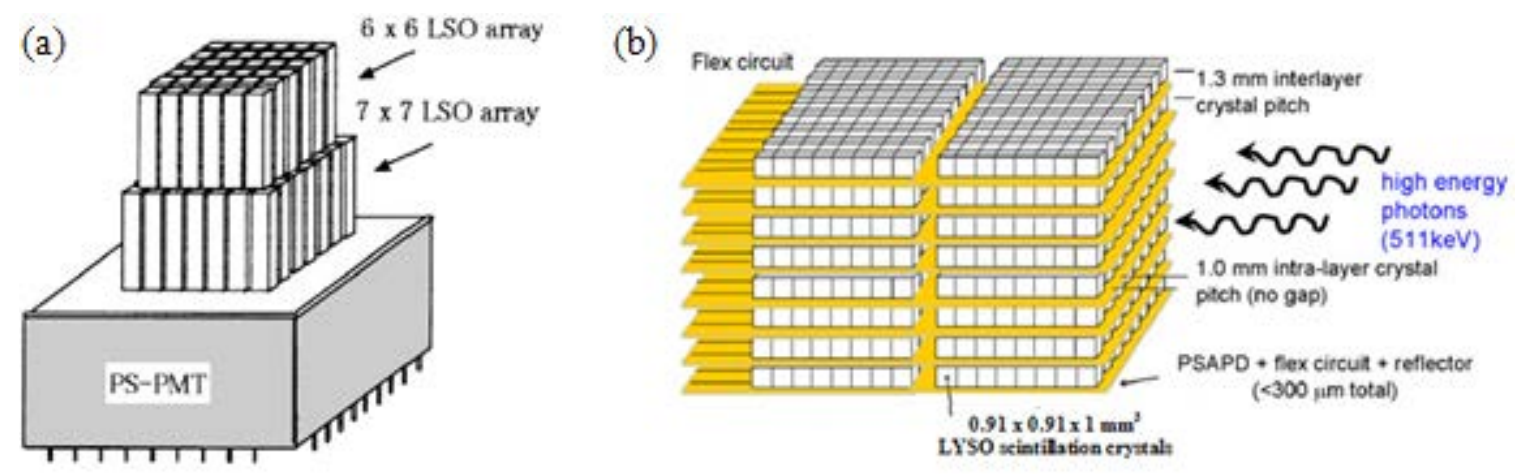

Fig. 4.8 (a) Schematic diagram of a discrete DOI encoding two layer relative offset detector (Liu, 183). (b) Schematic diagram of an eight layer discrete DOI encoding detector based on sub-millimeter scintillation crystals coupled to thin PSAPDs and providing direct DOI sampling (Vandenbroucke, 5897).

\subsubsection{Direct DOI Encoding Detector}

The aforementioned discrete DOI encoding detectors estimate DOI indirectly. For the phoswich approach DOI information depends on subsequent pulse shape discrimination analysis while for the light sharing and relative offset approaches DOI information depends on neighboring scintillation array structure surrounding the initial crystal of interaction. A novel discrete DOI encoding detector first proposed by Levin et al. (Levin, 2236-2243) removes the so to speak middle man and provides direct DIO sampling, thus enhancing overall DOI performance. The detector is comprised of two modules mounted on a common flex circuit one in front of the other. Each module is comprised of an array of scintillation crystals coupled to a thin PSAPD. The scintillation crystals are mounted such that their long side is coupled to the face of the PSAPD thus resulting in a light collection efficiency of $\geq 90 \%$. Ultimately it is proposed the detector consist of sixteen total detector modules, stacked atop one another in an $8 \times 2$ configuration. The most recent results, as reported by Vandenbroucke et al., focus on the performance of one layer of the detector shown in Fig. 4.8b. Utilizing two 8 x 8 arrays of 0.91 x 0.91 x $1 \mathrm{~mm}^{3}$ optically isolated LYSO scintillation crystals each coupled to a separate thin PSAPD, average energy resolution for "face on" and "edge on" incident radiation was 13.5\% FWHM and 14.6\% FWHM respectively. Average $\mathrm{x}-\mathrm{y}$ spatial resolution, when measured in the "edge on" configuration, was $.837 \mathrm{~mm}$ FWHM (Vandenbroucke, 5895-5911). DOI resolution was not explicitly quoted, however, as DOI resolution is a function of the lateral length of the scintillation crystal of interaction and nearly cubic scintillation crystals were used, spatial x-y resolution and depth resolution should be similar. While these results are impressive, there are serious questions concerning performance, construction viability, and increased cost when more than one layer is attempted. 


\subsubsection{Continuous DOI Encoding}

Continuous DOI encoding is achieved through a panoply of multifarious detector module architectures and DOI information extraction methods. However, in general, as opposed to discrete DOI encoding continuous DIO encoding results in increased DOI sensitivity due to the unmitigated sampling of DOI information. It should also be mentioned that certain continuous DOI encoding detectors, such as monolithic crystal detectors discussed below, exhibit appreciable timing performance and light collection efficiently as compared to the multi-layer discrete DOI encoding detectors discussed above.

\subsubsection{Dual Ended Readout Detectors}

One such detector geometry providing continuous DOI measurement is the dual-ended readout design first proposed by Hamamatsu Photonics K. K. (Shimizu, 717-721). The dual ended readout design employs two photodetectors each of which is optically coupled to the opposing axial ends of either a pixelated scintillation crystal array (Fig. 4.9) or a monolithic scintillation crystal as discussed above. The dual ended readout approach is appealing, especially when compared to a number of discrete DOI encoding approaches discussed above, in that calculations to extract DOI (discussed below) do not require pulse shape discrimination, scintillation crystal arrays are less complex thus reducing the cost as well as the mechanical precision necessary to manufacture them, extremely accurate alignment to prevent pixel overlap is not necessary, and, as with most continuous DOI encoding detectors, DOI resolution is improved. There are however drawbacks which include increased photodetector, amplifier, and readout electronics costs.

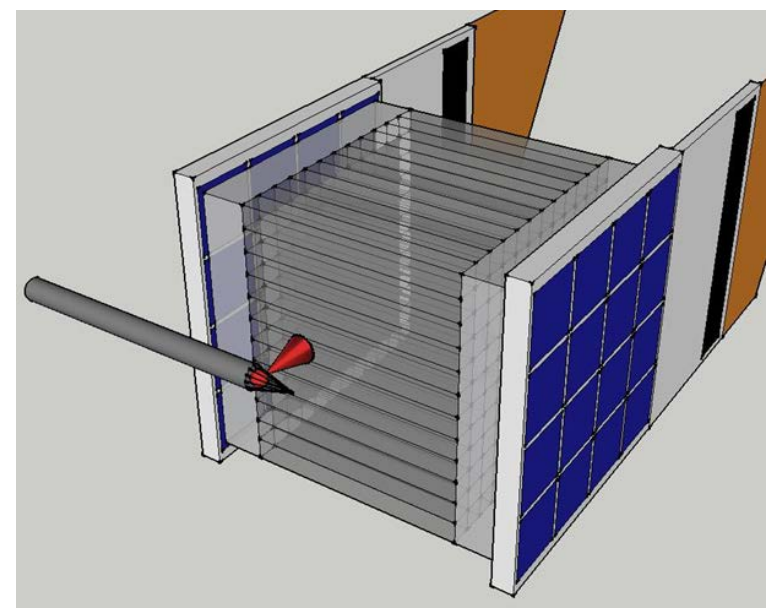

Fig. 4.9 Schematic diagram of a pixelated scintillation array coupled to light spreader windows and photodectors on opposing axial ends. The detector is irradiated by a collimated narrow beam needle source at various positions along the axial length of the scintillation crystals. The signal asymmetry in the readout from both photodetectors is used to determine DOI.

In the pixelated approach the axial point of incident radiation interaction along the length of a scintillation crystal (DOI) is estimated on an event by event and crystal by crystal basis utilizing the ratio of the magnitude of incident light on one photodetector to the sum magnitude of incident light on both photodetctors. In other words, the voltage output corresponding to the integration of the sum analog pulse of one photodetector (PD1) divided by the total voltage output corresponding to the aggregate integration of the sum analog pulses from both photodetectors (PD1+PD2):

$$
\text { DOI }=\mathbf{k} \frac{\mathrm{PD} 1}{(\mathrm{PD} 1+\mathrm{PD} 2)}+\mathrm{C}=\mathbf{k} * \text { DOI Ratio }+\mathrm{C}
$$


with $\mathrm{k}$ equal to calibration factor and $\mathrm{C}$ equal to a constant. Once multiplied by a conversion factor (further discussed in chapter 6) the voltage ratio yields the axial coordinate of interaction, while the total voltage output, proportional to the sum scintillation photon yield, is used to estimate the energy of the incident radiation. For multiple events at a given axial coordinate, a subsequent distribution of estimated depths of interaction and energy are generated with the full-width-half-maximum (FWHM) of each corresponding to the detectors depth and energy resolution respectively.

In order for the fractional output method to yield viable depth of interaction information, the magnitude of light incident on both axially opposing photodetectors must be depth dependent. Scintillation photons generated at the axial point of incident radiation interaction propagate in opposite directions via internal reflection towards both photodetectors. The magnitude or intensity of scintillation photons that reaches both photodetectors is governed by equation 1.21. As discussed in chapter 2, one of the properties defining an optimum scintillation crystal includes transparency to the wavelength of its own emitted scintillation photons. This stipulation requires the bulk attenuation coefficient $\mu$ of equation 1.21 to be equal to zero for the scintillation crystal, thus rendering the magnitude of light incident on either photodetector depth independent. However, the crystal surfaces not in contact with a photodetector may undergo a roughening treatment, of which results in an effective attenuation coefficient $\mu_{\mathrm{e}}$ greater than zero along the surfaces of the crystal. Consequently, while scintillation photons ideally remain transparent to the bulk of the scintillation crystal, a resultant attenuation of scintillation photons at each boundary reflection along the path length of the scintillation crystal is observed and magnitude of light incident on either photodetector becomes depth dependent. As the effective attenuation coefficient is increased, via increased surface roughening, DOI sensitivity, and thus DOI resolution, are increased. For small values of $\mu_{\mathrm{e}}$ the magnitude of light incident on either photodetector varies linearly with DOI, however, for large values of $\mu_{\mathrm{e}}$ the magnitude becomes nonlinear with DOI. This nonlinearly results in a degraded capacity to determine the energy of an event, resulting in reduced energy resolution along the axial length of the scintillation crystal, and thus, detector wide degradation of energy resolution. Along with the nonlinear variations, decreases in light yield as a result of decreasing the cross-sectional area of scintillation crystals in pursuit of increased $x-y$ resolution, can compound the degradation in energy resolution. Therefore, when implementing the pixelated dual-ended readout design, an exercise in compromise between DOI resolution, energy resolution, and in some cases $\mathrm{x}-\mathrm{y}$ resolution, must be undertaken.

It should also be noted that nonlinearity in light yield can also lead to non-uniformity in photodetector signal asymmetry. As a result, the procedure required to properly calibrate the DOI response of a detector module, and thus optimize DOI performance, is increased in complexity. Recent studies by Taghibakhsh et al. attempt to address the deleterious effects associated with nonlinear light yield through the use of a single parameter adaptive equation. The equation empirically corrects the height of the output analog pulses to enable a global energy spectrum, as well as, restores the uniformity of signal asymmetry along the axial length of a scintillation crystal (Taghibakhsh, 2151-2154). Examples of the pixelated dual ended approach are as follows.

Utilizing a 4 x 8 array of $3 \mathrm{~mm}$ x $5 \mathrm{~mm}$ x $50 \mathrm{~mm}$ BGO scintillation crystals with coarsely ground sides and two position sensitive PMTs (Hamamatsu R2487), the aforementioned initial Hamamatsu design achieved an approximate DOI resolution of $9.5 \mathrm{~mm}$ FWHM across all pixels at each of five incremental $10 \mathrm{~mm}$ irradiation position along the axial length of the detector (Shimizu, 717-721). Subsequent variations of the initial Hamamatsu design were developed, however initial gains in DOI performance were modest. Examples include studies by Moses et al., in which a PMT was used to readout one end of an $8 \times 8$ BGO array and a 64 pixel photodiodes (PD) was used to readout the other (Moses, 1441-1445), as well as subsequent follow up studies by both Huber et al. and Wang et al. (Huber, 684-688; Wang, 775-781). 
Significant increases in the performance of the dual-ended readout design were marked by the advent and, adoption of, the position sensitive avalanche photodiode (PSAPD) (Shah, 1687-1692). Examples include studies by Dokhale and St. James, as well as multiple studies by Yang; all at UC Davis. Utilizing a $7 \times 7$ array of saw cut $1 \times 1 \times 20 \mathrm{~mm}^{3}$ LSO scintillation crystals separated by a milti-layer polymer reflector and coupled to $8 \times 8 \mathrm{~mm}^{2}$ PSAPDs at both ends, studies by Dokhale et al. demonstrated a DOI resolution of 3-4 mm FWHM along the entire length of crystal and for various crystals occupying different locations within the array. Energy resolution was shown to vary between $13 \%$ and 15\% FWHM for various DOI positions demonstrating nearly uniform light collection along the length of the crystals (Dokhale, 4293-4304). Studies by Yang et al. explored the use of various $20 \mathrm{~mm}$ long LSO scintillation arrays (polished and unpolished) with individual pixel sizes of 1.0, 1.3, and $1.5 \mathrm{~mm}$ separated by a $64 \mu \mathrm{m}$ thick polymer film and coupled to $8 \times 8 \mathrm{~mm}^{2}$ PSAPDs at both ends. The best DOI resolution was achieved for the $1 \mathrm{~mm}$ array with unpolished crystals and when averaged over all crystals and all depths equaled $3.9 \mathrm{~mm}$ FWHM. The worst DOI resolution, $16.5 \mathrm{~mm}$ FWHM, was for the single polished array $(1.3 \mathrm{~mm})$ and clearly demonstrates the marginal dependence of light yield with depth for polished crystals. Energy resolution averaged over all crystals and all depths arrays ranged from $14.7 \%$ to $15.4 \%$ for the arrays tested (Yang, 2131-2142).

Further studies by Yang et al. include the investigation of two methods, a linear detector calibration and a linear crystal calibration, for mapping the DOI response for a large number of detector elements comprising a scanner (Yang, 433-445), as well as, the deployment of a prototype PET scanner comprised of two detector plates each of which is comprised of four detector modules. Each detector module was composed of $7 \times 7$ array of $0.9225 \times .09225 \times 20 \mathrm{~mm}^{3}$ LSO scintillation crystals separated by a $65 \mu \mathrm{m}$ thick specular reflector and coupled to $8 \times 8 \mathrm{~mm}^{2}$ PSAPDs at both ends. Studies on an individual detector module yielded an average DOI resolution of approximately $2 \mathrm{~mm}$ FWHM when averaged over 21 interior crystals and all depths and an energy resolution of 15\%. Subsequent phantom studies performed with the scanner showed DOI measurements significantly improved the reconstructed spatial resolution (Yang, 1132-1140).

Recent studies by St. James characterize the performance of finely pixelated sub-mm scintillation arrays of which exhibit encouraging performance. Employing a 10 x 10 array of 0.7 x 0.7 x $20 \mathrm{~mm}^{3}$ LSO scintillation crystals with either a $65 \mu \mathrm{m}$ thick specular, or a $50 \mu \mathrm{m}$ thick diffuse reflector, and coupled to $8 \times 8 \mathrm{~mm}^{2}$ PSAPDs at both ends. On the flood histogram, the specular array demonstrated poor crystal identification near the edges across the entire temperature range tested while the diffuse reflector array was able to clearly identify all the crystals at temperatures of $0^{\circ} \mathrm{C}$ and lower. DOI resolution averaged over crystals over all depths, as well as energy resolution averaged over all crystals and all depths, equaled $2.1 \mathrm{~mm}$ FWHM and 29\% for the specular array and $1.7 \mathrm{~mm}$ FWHM and 31\% for the diffuse array at $0^{\circ} \mathrm{C}$. Studies were also performed on both a specular and diffuse $13 \times 13$ array of $0.5 \times 0.5 \times 20$ $\mathrm{mm}^{3}$ LSO scintillation crystals, however, crystal identification, DOI resolution, and energy resolution were worse (St. James, 4605-4619). This is most likely due to the nonlinearity and diminished light yield of finely pixelated scintillation crystals discussed above.

Concurrent to the use of PSAPDs in the dual ended readout design are the use of CMOS solid state photomultipliers (SSPM), as well as next generation silicon photomultipliers (SIPM). Studies by Dokhale et al. employing a $4 \times 4$ array of $1.43 \times 1.43 \times 20 \mathrm{~mm}^{3}$ saw cut LYSO scintillation crystals optically isolated by a 3M reflector film and coupled a 4 x 4 array of $1.5 \times 1.5 \mathrm{~mm}^{2}$ CMOS SSPM elements at both ends demonstrated promising initial performance results for a dual ended readout CMOS SSPM approach. All pixels in the flood histogram were clearly visible, the DOI resolution measured for each crystal and averaged over all depths ranged from $2.3 \mathrm{~mm}$ to $2.9 \mathrm{~mm}$, and the depth independent energy resolution measured for each crystal ranged from 13\% to 17\% FWHM (Dokhale, 2809-2812). Recent studies by Taghikhsh et al. investigate the applicability of SiPMs to the dual ended readout approach, and while the studies were little more than an investigative proof of concept utilizing only a 
single scintillation crystals, the results proved encouraging. Studies evolved the use of either a saw cut or polished $2 \times 2 \times 20 \mathrm{~mm}^{3}$ LYSO scintillation crystal coupled to a $2.1 \times 2.1 \mathrm{~mm}^{2}$ SiPM at both ends. The signal asymmetry between SiPMs for both the saw cut and the polished crystals irradiated from one end demonstrate a much wider profile for the saw cut crystal and thus increased DOI sensitivity and higher DOI resolution. DOI resolution for the saw cut crystal was estimated to be $2.1 \mathrm{~mm}$ FWHM while the DOI resolution for the polished crystal was estimated to be $9.0 \mathrm{~mm}$ FWHM. Energy resolution for the saw cut crystal varied from 14\% FWHM at either end of the crystal to 19\% FWHM at the center. Energy resolution for the polished crystal proved depth independent and remained constant at 14\% FWHM (Taghibakhsh, 2821-2826; Taghibakhsh, S250-S254).

\subsubsection{Monolithic Crystal Detectors}

Another such detector design providing continuous DOI measurement are detectors employing a continuous monolithic scintillation crystal coupled to one or more photodetectors. The interest in monolithic crystal based detectors is on the rise as such detectors seek to address various performance issues inherent to pixelated crystal based detectors. One issue often experienced by detectors employing a finely pixelated scintillation array is decreased light yield (as discussed in the dual ended readout section) thus resulting in degraded energy resolution. Light collection efficiency is also problematic as discussed in the discrete multi-layer DOI encoding section above. Furthermore, a second issue stems from the necessity to optically isolate each pixel in an array in order to minimize light sharing and crosstalk. This is achieved by placing an intracrystal reflector between each individual pixel in an array. However, by doing so the dead space of the scintillation array is increased and thus overall detector sensitivity is decreased. In addition, a third issue often associated with pixelated scintillation arrays involves cost and labor. As the cross sectional area of pixels in an array is decreased, and or the intricacy and complexity of design of an array is increased, not only does mechanical precision to manufacture such arrays increase, so does the cost of doing so. Detectors employing monolithic scintillation crystals seek to offer a solution to these issues.

The monolithic crystal approach is contingent on creating look-up tables (LUT) to map the light distribution corresponding to various point of interaction within the crystal. The tables are then used as a reference against collected light signals to determine the 3-dimensional coordinate of incident radiation interaction within the crystal. However, based on the data acquisition and analysis technique employed monolithic scintillation crystal detectors can be divided into two categories.

First, the direct DOI approach, as is illustrated in studies by Ling et al. One quarter of a detector consisting of a $50 \times 50 \times 8 \mathrm{~mm}^{3}$ LYSO monolithic scintillation crystal coupled to a $52 \mathrm{~mm}^{2}$ flat panel PMT was irradiated via an electronically collimated $511 \mathrm{keV}$ gamma beam at various points on the crystal surface. Subsequent light distributions were recorded for both a normal and a $45^{\circ}$ angle of gamma incidences. Symmetry was then use to generate the detector wide statistically based positioning look-up tables corresponding to light distributions across the x-y plane of two DOI regions separated by $4.24 \mathrm{~mm}$. Utilizing a maximum-likelihood positioning algorithm it was found that under performance evaluation testing the DOI region misclassification rate was approximately 25\%. Even so, when compared to detector performance corresponding to only one DOI region (the entire crystal), intrinsic spatial resolution was shown to be maintained in the center of the crystal $(1.27 \mathrm{~mm}$ in $\mathrm{x}, 1.30 \mathrm{~mm}$ in y) and significantly improve in the corners (1.6 $\mathrm{mm}$ in $\mathrm{x}, 1.79 \mathrm{~mm}$ in y) (Ling, 2213-2228).

The second monolithic crystal category, an indirect DOI approach, is illustrated in studies by both Maas et al. and Schaart et al. Studies by Maas et al. employ variously sized LSO or LYSO monolithic scintillation crystals with various surface treatments and coupled to either one or two $8 \times 4$ element $\mathrm{APD}(\mathrm{s})$. The APD(s) are couple to either the front, the back, or both opposing ends of the crystal. The front surface of the crystal is irradiated at various $\mathrm{x}-\mathrm{y}$ coordinates and at various angles via an 
electronically collimated gamma beam in order to document the corresponding light distributing (Fig. 4.10).

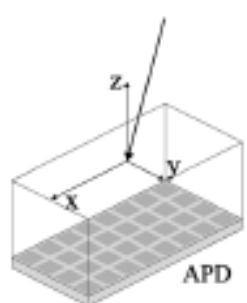

(a)

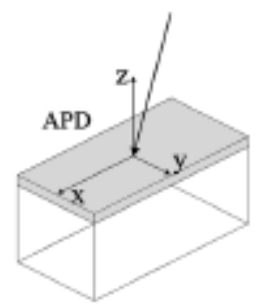

(b)

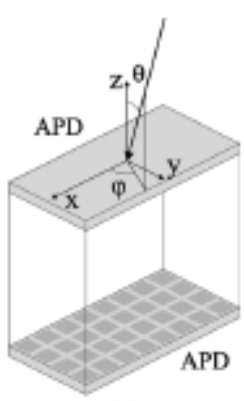

(c)

Fig. 4.10 Schematic diagram of monolithic scintillation crystal based detectors with (a) back side readout, (b) front side readout, and (c) dual ended readout (Maas, 1073). Estimating the entry point of incident radiation provides an intrinsic correction for DOI.

Using a statistical nearest neighbor algorithm a reference set of look-up tables is generated for each particular angle of incidence with the entry point of subsequent incident annihilation photons estimated by comparing the measured light distribution with the reference set. Estimating the entry point rather than the point of interaction within the crystal has the advantage of eliminating parallax error even though the DOI is not explicitly determined (Maas, 1896). Reconstructed spatial resolution for the single ended readout of a $20 \times 10 \times 10$ monolithic crystal was consistently better when read out from the front side as opposed to the back side of the detector as a majority of interactions occur in the first $5 \mathrm{~mm}$ of the crystal. The best reconstructed spatial resolution, $1.96 \mathrm{~mm}$, was obtained for a $20 \mathrm{x} 10 \mathrm{x} 10$ polished LYSO crystal with front side readout. Experiments with the $20 \mathrm{~mm}$ thick crystals read out on both sides yielded reconstructed spatial resolution only slightly worse than the best $10 \mathrm{~mm}$ thick crystal. Thus increasing the crystal thickness significantly improves the detector efficiency without compromising resolution (Maas, 1071-1077). Follow up studies by Maas further explore the benefits of the dual ended readout. In these studies a $20 \mathrm{~mm}$ thick trapezoidal or rectangular monolithic LYSO scintillation crystal is coupled to a PSAPD on both ends. Both geometries yield an energy resolution of approximately $11 \%$ FWHM, while the rectangular geometry yielded the best spatial resolution; $1.9 \mathrm{~mm}$ near the edge of the crystal and getting better towards the center (Maas, 1893-1908).

Studies by Schaart et al. follow in the footsteps of Maas, however next generation $4 \mathrm{x} 4$ element SiPM arrays are employed and coupled to either the front or back face of a $13.2 \times 13.2 \times 10 \mathrm{~mm}^{3} \mathrm{LYSO}$ monolithic scintillation crystal. Measurements performed using the same method as Maas confirms the increased performance of the front side readout versus the back side read out. Spatial resolution of $\leq 1.6$ mm FWHM was obtained while energy resolution was between 12\% and 14\% FWHM (Schaart, 35813585; Schaart, 3501-3512).

\subsubsection{Phosphor Coated Scintillator Detector}

A novel DOI detector providing continuous encoding was recently proposed by Du et al. and is based on coating the far ends of scintillation crystals with a thin layer of phosphor powder and coupling the near ends of the crystals to a single photodetector. The phosphor powder absorbs a fraction of the scintillation photons generated in the bulk scintillation crystal and subsequently emits wavelength shifted photons with a longer decay time. The fraction of scintillation photons absorbed by the phosphor powder is dependent on the proximity of scintillation photon generation to the coated area. As a result, the constituent light pulse is a convolution of scintillation photons with both short and long decay times with the ratio of the two determining the shape of the analog output pulse of the photodetector. Therefore, like 
the phoswich approach, DOI information can be extracted via pulse shape discrimination of the output analog pulse. However, unlike the phoswich approach, DOI encoding is continuous. Studies by Du utilize a 4 x 4 array of unpolished $1.5 \times 1.5 \times 20 \mathrm{~mm}^{3}$ LSO crystals half coated on the far end with YGG phosphor powder and coupled on the near end to a single PMT. Unpolished crystals were used to facilitate light loss as described in the dual ended readout section. Consequently energy resolution, as measured for a single centrally located crystal, ranged from 26.9\% FWHM to 33.0\% FWHM over various depths. DOI resolution averaged over all crystals and all depths was estimated to be $8.0 \mathrm{~mm}$ FWHM (Du, 1757-1771). While not spectacular, the main advantage to this approach is the ease of implementation and cost savings, of which are on par with a number of discrete DOI encoding approaches, while still offering continuous DOI readout.

\section{$\underline{4.5 ~ O v e r v i e w}$}

Position emission tomography is based on the indirect detection of coincident annihilation photons. This is achieved through the use of scintillation based detector modules typically oriented in a ring geometry. However, as the ring geometry is not always practical and or cost efficient. As a result there is an increasing interest in dedicated organ, as well as purpose specific PET probes and scanners; many of which are incorporating depth of interaction capability. The extraction of DOI information and incorporation into a PET detector's reconstruction algorithm can appreciably reduce parallax error and provide nearly isotropic and uniform spatial resolution throughout the entire field of view. Currently there is a plethora of DOI encoding detector modules offering both discrete and continuous DOI encoding. With each DOI approach there is a trade off between DOI resolution and other detector performances. However, when considering small dedicated organ probes the dual ended readout approach appears to offer the best compromise between over all performance, cost, and logistical implantation. As such, due to the archaic nature surrounding the current diagnosis and treatment of prostate cancer further discussed in chapter five, a high resolution dual ended readout DOI PET detector proposed for prostate imaging has been implemented at West Virginia University. Utilizing the DOI module as an endorectally inserted probe, it is proposed that the incorporation of DOI information will vastly improve the spatial resolution of the prostate thus aiding in diagnosis and biopsy guidance of prostatic carcinomas. A full review of the DOI module's performance will be presented in chapter six.

\section{References}

Adaptive I/O Technologies, Inc. http://www.ait-instruments.com/.

Belcari, N., F. Attanasi, V. Rosso, and A. Del Guerra, "Staggered double-layer array crystals for the reduction of the depth of interaction uncertainty in in-beam PET: A preliminary study.” Nuclear Instruments and Methods in Physics Research, vol. 617, issue 1, pp. 246-247, 2010.

Casey, M. E., and R. Nutt, "A multicrystal two dimensional BGO detector system for positron emission tomography.” IEEE Transactions on Nuclear Science, vol. 33, issue 1, pp. 460-463, 1986.

Chandrikamohan, P, and T. A. DeVol, "Comparison of Pulse Shape Discrimination Methods for Phoswich and CsI:Tl Detectors.” IEEE Transactions on Nuclear Science, vol. 54, issue 2, pp. 398-403, 2007.

Dokhale, P. A., R. W. Silverman, K. S. Shah, R. Grazioso, R. Farrell, J. Glodo, M. A. McClish, G. Entine, V. H. Tran, and S. R Cherry, "Performance measurements of a depth-encoding PET detector module based on position-sensitive avalanche photodiode read-out.” Physics in Medicine and Biology, vol. 49, issue 18, pp. 4293-4304, 2004. 
Dokhale, P., C. Staples, J. Christian, Y. Yang, S. Cherry, W. Moses, and K. Shah, "Performance Measurements of a SSPM-LYSO-SSPM Detector Module for Small Animal Positron Emission Tomography.” 2009 IEEE Nuclear Science Symposium Conference Record, pp. 2809-2812, 2009.

Du, Huini, Y. Yang, J. Glodo, Y. Wu, K. Shah, and S. R. Cherry, “Continuous depth-of-interaction encoding using phosphor-coated scintillators.” Physics in Medicine and Biology, vol. 54, issue 6, pp. 1757-1771, 2009.

Düppenbecker, P. M., S. Lodomez, R. Haagen, P. K. Marsden, and V. Schulz, "Investigation of a Sub-Millimeter Resolution PET Detector with Depth of Interaction Encoding Using Digital SiPM Single Sided Readout.” 2008 IEEE Nuclear Science Symposium Conference Record, pp. 2252-2253, 2008.

Eriksson, L., C. L. Melcher, M. Zhuravleva, M. Eriksson, H. Rothfuss, and M. Conti, "Phoswich solutions for the PET DOI problem.” Nuclear Instruments and Methods in Physics Research A, vol. 648, supplement 1, pp. S288-S292, 2011.

General Electric, http://www.gehealthcare.com/euen/molecularimaging/products/pet_ct_imaging/discovery_petct690/.

General Electric, http://www.gmecorp-usa.com/IM/NM/PS/GE/ADVANTAGE/specs-01.pdf.

Hamamatsu Photonics K. K. http://sales.hamamatsu.com/.

Huber, J. S., W. S. Choong, W. W. Moses, J. Qi, J. Hu, G. C. Wang, D. Wilson, S. Oh, R. H. Huesman, S. E. Derenzo, and T. F. Budinger, "Initial Results of a Positron Tomograph for Prostate Imaging." IEEE Transactions on Nuclear Science, vol. 53, issue 5, pp. 2653-2659, 2006.

Huber, J. S., W. W. Moses, M. S. Andreaco, and O. Petterson, “A LSO Scintillator Array for a PET Detector Module with Depth of Interaction Measurement.” IEEE Transactions on Nuclear Science, vol. 48, issue 3, pp. 684-688, 2001.

Inadama, N., H. Murayama, M. Hamamoto, T. Tsuda, Y. Ono, T. Yamaya, E. Yoshida, K. Shibuya, F. Nishikido, J. Kikuchi, and T. Doke, "8-layer DOI encoding of 3-Dimensional Crystal Array.” 2005 IEEE Nuclear Science Symposium Conference Record, pp. 2766-2769, 2005.

Ito, M., J. S. Lee, S. I. Kwon, G. S. Lee, B. Hong, K S. Lee, K. S. Sim, S. J. Lee, J. T. Rhee, and S. J. Hong, “A Four-Layer DOI Detector with a Relative Offset for Use in an Animal PET System.” IEEE Transactions on Nuclear Science, vol. 57, issue 3, pp. 976-981, 2010.

Ito, Mikiko, S. J. Hong, and J. S. Lee, "Positron Emission Tomography (PET) Detectors with Depth-of-Interaction (DOI) Capability.” Biomedical Engineering Letters, vol. 1, issue 2, pp. 70-81, 2011.

Jung, J. H., Y. Choi, Y. H. Chung, O. Devroede, M. Krieguer, P. Bruyndonckx, and S. Tavernier, “Optimization of LSO/LuYAP phoswich detector for small animal PET." Nuclear Instruments and Methods in Physics Research A, vol. 571, issue 3, pp. 669-675, 2007.

Kang, J., Y. Choi, J. H. Jung, W. Hu, H. K. Lim, and B. T. Kim, “A Depth-Encoding PET Detector Inserting Glass Plate Between Layers.” 2011 IEEE Nuclear Science Symposium Conference Record, pp. 2859-2863, 2011.

Karp, J. S., and G. Muehllehner, “Performance of a position-sensitive scintillation detector.” Physics in Medicine and Biology, vol. 30, issue 7, pp. 643-655, 1985.

Kitamura, K. A. Ishikawa, T. Mizuta, T. Yamaya, E. Yoshida, and H. Murayama, "Detector normalization and scatter correction for the jPET-D4: A 4-layer depth of interaction PET scanner.” Nuclear Instruments and Methods in Physics Research A, vol. 571, issue 1-2, pp. 231-234, 2007. 
Levin, C. S., "Design of a High-Resolution and High-Sensitivity Scintillation Crystal Array for PET with Nearly Complete Light Collection.” IEEE Transactions on Nuclear Science, vol. 49, issue 5, pp. 2236-2243, 2002.

Levin, C. S., "New Imaging Technologies to Enhance the Molecular Sensitivity of Positron Emission Tomography." Proceedings of the IEEE, vol. 96, issue 3, pp. 448, 2008.

Ling, T., T. K. Lewellen, and R. S. Miyaoka, "Depth of interaction decoding of a continuous crystal detector module.” Physics in Medicine and Biology, vol. 52, issue 8, pp. 2213-2228, 2007.

Liu, H., T. Omura, M. Watanabe, and T. Yamashita, "Development of a depth of interaction detector for $\gamma$-rays." Nuclear Instruments and Methods in Physics Research A, vol. 459, issue 1-2, pp. 182-190, 2001.

Maas, M. C., D. J. van der Laan, D. R. Schaart, J. Huizenga, J. C. Brouwer, P. Bruyndonckx, S. Léonard, C. Lemaître, and C. W. E. van Eijk, "Experimental Characterization of Monolithic-Crystal Small Animal PET Detectors Read Out by APD Arrays.” IEEE Transactions on Nuclear Science, vol. 53, issue 3, pp. 1071-1077, 2006.

Maas, M. C., D. R. Schaart, D. J. van der Laan, P. Bruyndonckx, C. Lemaître, F. J. Beekman, and C. W. E. van Eijk, "Monolithic scintillator PET detectors with intrinsic depth-of-interaction correction.” Physics in Medicine and Biology, vol. 54, issue 7, pp. 1893-1908, 2009.

Moses, W. W., and S. E. Dorenzo, "Design Studies of a PET Detector Module using A PIN Photodiode to Measure Depth of Interaction.” IEEE Transactions on Nuclear Science, vol. 41, issue 4, pp. 1441-1445, 1994.

Nishikido, F., N. Inadama, K. Shibuya, E. Yoshida, T. Yamaya, I. Oda, K. Kitamura, and H. Murayama, "Four-layer DOI-PET detector with a silicon photomultiplier array.” .” 2008 IEEE Nuclear Science Symposium Conference Record, pp. 3923-3925, 2008.

Phelps, Michael E., ed. PET: Physics Instrumentation, and Scanners. New York: Springer Science + Business Media, Inc., 2006.

Philips Scientific. http://www.phillipsscientific.com/.

Philips,

http://www.healthcare.philips.com/us_en/products/nuclearmedicine/products/geminitf/index.wpd\#\&\&/wEXAQ UOY3VycmVudFRhYlBhdGgFFkRldGFpbHM6U3BlY2lmaWNhdGlvbnOMsm5HSTlfUhiZBla88BAYqeUic $\underline{w}==$.

Proffitt, J., W. Hammond, S. Majewski, V. Popov, R. R. Raylman, A. G. Weisenberger, and R. Wojcik, “A flexible high-rate USB2 data acquisition system for PET and SPECT imaging.” IEEE Nuclear Science Symposium Conference Record, vol. 5, pp. 2971-2975, 2005.

Proteus Inc. http://www.apace-science.com/proteus/yap.htm.

Raylman, R.R., S. Majewski, B. Kross, V. Popov, J. Proffitt, M. F. Smith, A. G. Weisenberger, and R. Wojcik, "Development of a dedicated positron emission tomography system for the detection and biopsy of the breast." Nuclear Instruments and Methods in Physics Research A, vol. 569, issue 2, pp. 291-295, 2006.

Rhodia Silicones. http://www.precisionconverting.com/optical.htm.

Rogers, J. G., “Testing an Improved Scintillation Camera for PET and SPECT.” IEEE Transactions on Nuclear Science, vol. 33, issue 1, pp. 519-522, 1986.

Rogers, J. G., D. P. Saylor, R. Harrop, X. G. Yao, C. V. M. Leitao, and B. D. Pate, "Design of an efficient position sensitive gamma ray detector for nuclear medicine.” Physics in Medicine and Biology, vol. 31, issue 10, pp. 1061-1090, 1986. 
Rohren, E. M., T. G. Turkington, and R. E. Coleman, “Clinical Applications of PET in Oncology.” Radiology, vol. 231, issue 2, pp. 305-332, 2004.

Safavi-Naeini, M., D. R. Franklin, M. Petasecca, M. L. F. Lerch, R. Kirkham, G. Moorhead, P. Dunn, G. De Geronimo, and A. B. Rosenfeld, "Preclinical Studies Using a Prototype High-Resolution PET System with Depth of Interaction.” 2011 IEEE Nuclear Science Symposium Conference Record, pp. 3305-3307, 2011.

Saha, Gopal B., Basics of PET Imagine: Physics, Chemistry, and Regulations. New York: Springer Science + Business Media, Inc., 2005.

Schaart, D. R., H. T. van Dam, S. Seifert, R. Vinke, P. Dendooven, H. Löhner, and F. J. Beekman, "SiPM-Array Based PET Detector with Depth-of-Interaction Correction.” 2008 IEEE Nuclear Science Symposium Conference Record, pp. 3581-3585, 2008.

Schaart, D. R., H. T. van Dam, S. Seifert, R. Vinke, P. Dendooven, H. Löhner, and F. J. Beekman, “A novel, SiPMarray-based, monolithic scintillator detector for PET.” Physics in Medicine and Biology, vol. 54, issue 11, pp. 3501-3512, 2009.

Shah, K. S., R. Farrell, R. Grazioso, E. S. Harmon, and E. Karplus, "Position-Sensitive Avalanche Photodiodes for Gamma-Ray Imaging.” IEEE Transactions on Nuclear Science, vol. 49, issue 4, pp. 1687-1692, 2002.

Shimizu, K., T. Ohmura, M. Watanabe, H. Uchida, and T. Yamashita, "Development of 3-D Detector System for Positron CT.” IEEE Transactions on Nuclear Science, vol. 35, issue 1, pp. 717-720, 1988.

Siemens, http://www.activexray.com/pdf/Siemens_Biograph.pdf.

Sparrow Corp. http://www.sparrowcorp.com/.

St. James, S., Y. Yang, Y. Wu, R. Farrell, P. Dokhale, K. S. Shah, and S. R. Cherry, "Experimental characterization and system simulations of depth of interaction PET detectors using $0.5 \mathrm{~mm}$ and $0.7 \mathrm{~mm} \mathrm{LSO}$ arrays." Physics in Medicine and Biology, vol. 54, issue 14, pp. 4605-4619, 2009.

Steun, M., G. Brandenburg, H. Larue, H. Saleh, E. Zimmermann, K. Ziemons, and H. Halling, "Pulse Shape Discrimination of LSO and PuYAP Scintillators for Depth of Interaction in PET.” IEEE Transactions on Nuclear Science, vol. 50, issue 3, pp. 344-347, 2003.

Taghibakhsh, F., A. Reznik, and J. A. Rowlands, "Silicon photomultipliers for positron emission tomography detectors with depth of interaction encoding capability.” Nuclear Instruments and Methods in Physics Research A, vol. 663, supplement 1, pp. S250-S254, 2011.

Taghibakhsh, F., C. S. Levin, and J. A. Rowlands, “Analytic Pulse Height Correction in Dual-Ended Readout PET Detectors.” 2010 IEEE Nuclear Science Symposium Conference Record, pp. 2151-2154, 2010.

Taghibakhsh, F., S. Cuddy, T. Rvachov, D. Green, A. Reznik, and J. A. Rowlands, "Detectors with Dual-Ended Readout by Silicon Photomultipliers for High Resolution Positron Emission Mammography Applications.” 2009 IEEE Nuclear Science Symposium Conference Record, pp. 2821-2826, 2009.

Tsuda, T., H. Murayama, K. Kitamura, T. Yamaya, E. Yoshida, T. Omura, H. Kawai, N. Inadama, and N. Orita, “A Four-Layer Depth of Interaction Detector Block for Small Animal PET.” IEEE Transactions on Nuclear Science, vol. 51, issue 5, pp. 2537-2542, 2004.

Vandenbroucke, A., A. M. K. Foudray, P. D. Olcott, and C. S. Levin, "Performance characterization of a new high resolution PET scintillation detector.” Physics in Medicine and Biology, vol. 55, issue 19, pp. 5895-5911, 2010. 
Vaquero, J. J., J. J. Sánchez, E. Lage, J. M. Udías, P. Guerra, and M. Desco, “Design of DOI PET detector modules using phoswich and SiPMs: First Results.” 2011 IEEE Nuclear Science Symposium Conference Record, pp. 3311-3313, 2011.

Wang, G. C., J. S. Huber, W. W. Moses, W. S. Choong, and J. S. Maltz, "Calibration of a PEM Detector with Depth of Interaction Measurement.” IEEE Transactions on Nuclear Science, vol. 55, issue 3, pp. 775-781, 2004.

Wei, Q., S. Wang, T. Ma, L. Lu, T. Dai, and Y. Liu, “Design of a high resolution phoswich PET detector.” 2011 IEEE Nuclear Science Symposium Conference Record, pp. 2874-2876, 2011.

Weisenberger, A. G., S. Majewski, D. Gilland, W. Hammond, B. Kross, V. Popov, J. Proffitt, J. McKisson, and M. F. Smith, "Implementation of a Mobile Cardiac PET Imager for the Emergency Room and Intensive Care Unit." 2007 Nuclear Science Symposium Conference Record, vol. 5, pp. 3705-3708, 2007.

Yamamoto, S., M. Honda, T. Oohashi, K. Shimizu, and M. Senda, "Development of a Brain PET System, PET-Hat: A Wearable PET System for Brain Research.” IEEE Transactions on Nuclear Science, vol. 58, issue 3, pp. 668673, 2011.

Yamaya, T., E. Yoshida, M. Satoh, T. Tsuda, K. Kitamura, T. Obi, T. Hasegawa, H. Haneishi, N. Inadama, S. Tanada, and H. Murayama, "The jPET-D4: Imaging Performance of the 4-Layer Depth-of-Interaction PET Scanner.” 2005 IEEE Nuclear Science Symposium Conference Record, pp. 1977-1980, 2005.

Yang Y. , P. A. Dokhale, R. W. Silverman, K. S. Shah, M. A. McClish, R. Farrell, G. Entine, and S. R. Cherry, "Depth of interaction resolution measurement for a high resolution PET detector using position sensitive avalanche photodiodes.” Physics in Medicine and Biology, vol. 51, issue 9, pp. 2131-2142, 2006.

Yang, Y., J. Qi, Y. Wu, S. St. James, R. Farrell, P. A. Dokhale, K. S. Shah, and S. R. Cherry, "Depth of interaction calibration for PET detectors with dual-ended readout by PSAPDs.” Physics in Medicine and Biology, vol. 54, issue 2, pp. 433-445, 2009.

Yang, Y., Y. Wu, J. Qi, S. St. James, H. Du, P. A. Dokhale, K. S. Shah, R. Farrell, and S. R. Cherry, “A Prototype PET Scanner with DOI-Encoding Detectors.” The Journal of Nuclear Medicine, vol. 49, issue 7, pp. 1132-1140, 2008. 


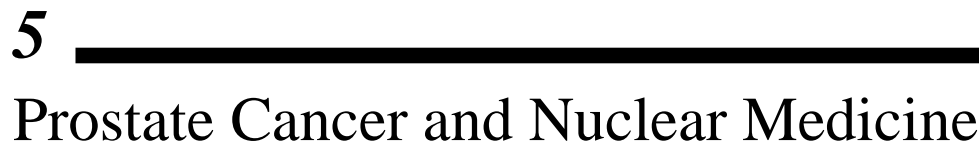

\section{$\underline{5.1 \text { Introduction }}$}

According to the National Cancer Institute (NCI), 241,740 new cases of prostate cancer will be diagnosed in the United States in 2012, of which, will be accompanied by 28,170 deaths due to the disease. Prostate cancer is the second most common form of cancer found in men in the United States, with only skin cancer being more prevalent (National Cancer Institute). Prostate cancer is also the second leading cause of cancer death in American men, with only lung cancer being more virulent (American Cancer Society (ACS)).

Worldwide, the most recent statistics for new cases of prostate cancer and deaths are from 2008. In that year, according to the GLOBOCAN Project, 899,102 new cases of prostate cancer were diagnosed which accounted for 13.6 percent of all new cancer cases in men. In that same year, 258,133 deaths due to prostate cancer were recorded which accounted for 6.1 percent of all deaths due to cancer (Ferlay). Since prostate cancer is most prevalent in men over the age of 50, and almost 2 out of 3 prostate cancers are found in men over the age of 65 (ACS), a global trend towards an aging population correlates to an expected rise in the number of new prostate cancer diagnoses as well as deaths due to prostate cancer. Taking into account current technology, practices, and procedures the GLOBOCAN Project predicts an approximately 21 percent increases in both new prostate cancer incidences as well as deaths by 2015 when compared to the statistics from 2008 (Ferlay). It should be noted that while the number of incidences is expected to rise, the screening of asymptomatic men becoming common practice in western countries, as well as the latent slow going nature of prostate cancer resulting in a majority of men dying with, rather than of, prostate cancer, is a source of inflation among the numbers.

In an effort to set the stage, as well as outline the motivation behind the studies presented in forthcoming chapter, this chapter will first present an overview of the prostate, prostate cancer, the current and most common early detection tests, and subsequent diagnosis of prostate cancer. Second, an in depth review of nuclear medicine's current role with regards to prostate cancer, especially in comparison to differing imaging modalities such as MRI, CT, and Transrectal Ultrasound, will be presented.

\section{$\underline{5.2}$ The Prostate}

The prostate is an accessory sex gland found only in men. The prostate begins to develop prior to birth and continues to grow until adulthood reaching a size roughly equivalent to a table tennis ball. During ejaculation, the prostatic acini (discussed below) are compressed by their surrounding fibromuscular stroma secreting prostatic fluid into the urethra. Secretions include proteolytic enzymes, prostatic acid phosphatase (PAP), prostate specific antigen (PSA), zinc, citrate, etc. which condition the urethral lumen prior to ejaculation. Prostatic secretions also aid in semen liquefaction post deposition in gelatinous form via seminal vesicle clotting enzymes (Aumúller, 127-133).

\subsubsection{Anatomy and Histology of the Prostate}

The prostate is located anteriorly to the rectum with its base abutting the bladder and seminal vesicles and its apex abutting the urethral sphincter (Fig 5.1). The parenchyma of the prostate is comprised of many acini, which are composed of four layers of cells. First, attached to the basal membrane are the basal 
(stem) cells, on top of which are early progenitor intermediate cells followed by late progenitor intermediate cells, and finally the luminal (secretory) cells (Algaba, 9-24). The acini empty into multiple ducts of which radiate from the verumontanum (veru); their point of termination. The veru, centrally situated within the prostate, is a small prominence in the prostatic urethra (the curved segment of the urethra which runs through the prostate from base to apex) that projects into the urethral lumen (Myers, 3). Flanking the veru are the ejaculatory ducts. As a whole, the prostate is invested by visceral fascia less its base and apex. Beneath the fascia and on the posterolateral surface of the prostate an intricate plexus of neurovascular bundles is found.

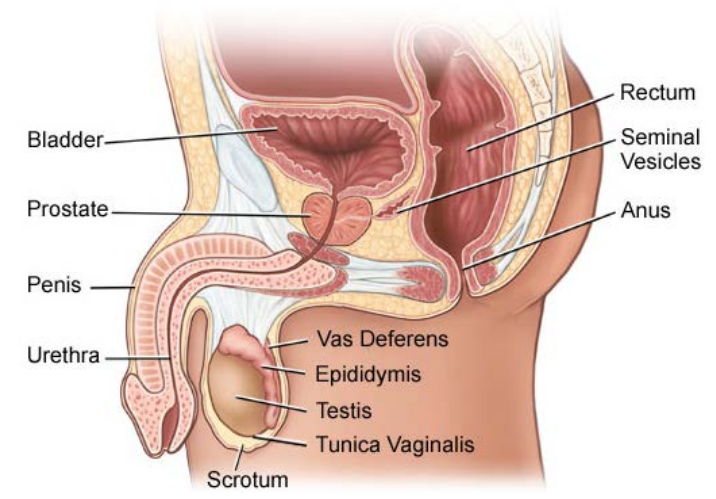

Fig 5.1 Male reproductive anatomy. The prostate is located below the bladder and in front of the rectum (Main Line Health).

While the architectural understanding of the prostate has evolved over the years, the zonal anatomy as defined by McNeal (McNeal, 340-345; McNeal 35-49) is widely accepted today. According to McNeal, while the prostate appears homogeneous on the macroscopic level, the prostate is divided into three distinct histological zones: the peripheral zone (70\% to $75 \%$ ), the central zone (20\% to $25 \%$ ), and the transition zone (5\% to 10\%) (Fig. 5.2).

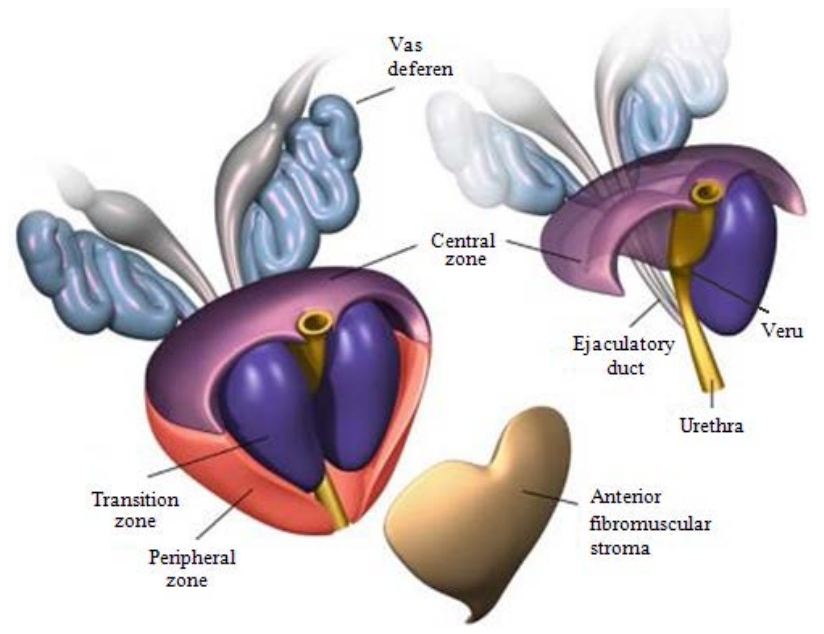

Fig. 5.2 Zonal anatomy of the prostate (Shah, 2).

The peripheral zone is characterized as a disc of tissue with ducts radiating laterally from the prostatic urethra lateral and distal to the veru. The central zone, having larger acini supported by a denser stromal tissue as compared to the peripheral zone, is characterized as an inverted cone shaped region, broad at the base and tapering towards the veru, whose ducts follow the encased ejaculatory ducts. The transition zone is characterized as two glandular groups bilateral to the urethra and proximal to the veru whose stromal tissue intermingles with anterior fibromuscular stromal tissue. The ducts in the transition zone are smaller compared to ducts in the other two zones and branch anteriorly from their singular 
urethral origin. According to McNeal it is also the transition zone that gives rise to benign prostatic hyperplasia (enlargement of the prostate).

\subsubsection{Prostate Cancer}

The vast majority of prostate cancers are adenocarcinomas with approximately $70 \%$ arising in the peripheral zone of the prostate (Kirby, 12). While the exact cause of prostate cancer is still unknown, risk factors such as age, race, nationality, family history, diet, and one's genes are thought to play a role. Prior to developing prostate cancer, pre-cancerous conditions such as proliferative inflammatory atrophy (PIA) (De Marzo, 1985-1992) and or prostatic intraepithelial neoplasia (PIN) (Bostwich, 1823-1836) are common precursors. It should however be mentioned that, in general, PIA, PIN, and prostate cancers are slow growing with autopsy studies of men deceased from causes unrelated to prostate cancer revealing that prostate cancer was found in 30 percent of men in their 50s and in 70 percent to 90 percent of men in their 80s to 90s worldwide (Breslow, 680-688; Guileyardo, 311-316; Bassett, 91-94; Sakr, 379-385). In many cases, the latent prostate cancer(s) found during autopsy had little to no impact on quality of life of the individual prior to death.

\subsection{Early Detection of Prostate Cancer}

Screening asymptomatic males for prostate cancer, while controversial, has become relatively common in western countries. According to the American Cancer Society males with an average risk for developing prostate cancer who are expected to live at least ten more years should consider screening beginning at age 50. For those individuals with a high to severe risk of developing prostate cancer screening should be considered beginning at ages 45 and 40 respectively (ACS). Typically screening involves both a digital rectal exam as well as one or more types of prostate-specific antigen (PSA) blood tests.

\subsubsection{Digital Rectal Exam}

A digital rectal exam involves a physician inserting the gloved tip of their index finger into a patents rectum in order to palpate the posterior surface of the prostate through the anterior rectal wall. A normal healthy prostate is roughly the size of a table tennis ball and is palpably smooth, mobile, symmetric, and of benign consistency (Myers, 4). A palpable cancerous lesion within the prostate is typically discernible as firm and asymmetric.

\subsubsection{PSA Blood Tests}

Prostate-specific antigen is a glycoprotein produced in the prostate. Healthy men typically have $4 \mathrm{ng} / \mathrm{mL}$ or less of PSA in their blood. While the level of PSA in the blood can be elevated in the absence of prostate cancer, for example, by $\mathrm{BPH}$, recent ejaculation, riding a bicycle, a urinary tract infection, prostatitis, and or age, men with a PSA level between 4 and $10 \mathrm{ng}$ per $\mathrm{mL}$ of blood have a 25 percent chance of having prostate cancer while men with PSA levels over $10 \mathrm{ng}$ per $\mathrm{mL}$ of blood have over a 50 percent chance of having prostate cancer (ACS).

While the PSA screening blood test can be beneficial in that it is a simple test that can detect potentially life threatening cancerous lesions early, it is hardly perfect. The European Randomized Study of Screening for Prostate Cancer found that PSA based screening reduced the mortality rate of prostate cancer by 20 percent, however 48 men would need to be treated for prostate cancer in order to prevent one death from prostate cancer. The study also found that PSA based screening was associated with a high risk of prostate cancer over diagnosis (Schröder, 1320-1328). More recently, in October 2011, the U.S. Preventive Service Task Force (USPSTF) controversially went so far as to issue a draft report, based 
in part on reviews done by Chou et al. and Lin et al. (Chou, 762-771; Lin 1-56), recommend against PSA based screening for asymptomatic males. In the draft, the USPSTF concluded that PSA based screening resulted in little to no reduction in prostate cancer mortality and often leads to further unnecessary test and treatments and even unnecessary radical prostatectomies resulting in possible impotence, incontinence, and appreciable decreases in quality of life. Once finalized, the recommendation will appear on the USPSTF website (see references).

It should be noted, that in an effort to improve the clinical significance of PSA based screening, PSA screening derivatives including percent free PSA, PSA velocity, PSA density, and age specific PSA have been developed in recent years. An overview of these derivatives can be found in the ACS booklet Prostate Cancer with a more in depth analysis found in the review by Bullock (Bullock, 196-211). The overall clinical value of PSA screening derivatives is currently still in dispute.

\section{$\underline{\text { 5.3.3 Biopsy and Current Prostate Gland Imaging Modalities }}$}

Regardless of screening method, if an individual's early detection tests lead their physician to suspect prostate cancer a prostate core needle biopsy will be preformed. This involves an urologist administering a local anesthetic and inserting an 18 gauge spring loaded needle through the rectal wall, or though a small incision in the perineum, into the prostate. When the needled is retracted a prostatic core tissue sample will be removed and sent to pathology to be graded using the Gleason scoring system (Gleason, 171-198). Typically, the prostate will be divided into a grid, with 8 to 18 core samples taken depending on the urologist's discretion. It has become common practice to use Transrectal Ultrasound (TRUS) to guide prostate biopsy with various protocols presented and discussed in the literature (Hodge, 66-70; Melchior, 463-471; Rodriguez, 2115-2120, Ravery, 298-303; Scherr, 18-31).

While TRUS allows an urologist to "see" the prostate, both to guide biopsy and asses prostate volume, newly suspected prostate cancer(s) that are most likely localized within the prostate are typically not ultrasonographically visible. Work done by Carter et al. supports this assertion and suggests that TRUS may not be a good method for detecting nonpalpable prostate cancer(s) localized within the prostate. Carter demonstrated that for 59 patients with palpable prostate caner TRUS had a sensitivity of only 52 percent and a specificity of 68 percent. (Carter, 1008-1010). An in vitro detection study of 19 prostatectomy specimens with histologically confirmed prostate cancer by Tayler et al. suggest that sonoelastography performs considerably better than standard ultrasound. Of seven lesions with volume $\geq$ $1 \mathrm{~cm}^{3}$ sonoelastography has a sensitivity of 71 percent and an accuracy of 55 percent. Of 22 lesions with volume $\leq 1 \mathrm{~cm}^{3}$ sonoelastography has a sensitivity of 41 percent and an accuracy of 34 percent (Taylor, 981-985). While this appears to be an improvement over standard ultrasound, the use of sonoelastography in a screening and diagnostic capacity requires further research.

In terms of guidance and visualization of internally localized prostate cancer(s), other modalities such as CT and MRI offer little improvement.

Utilizing CT, the prostate appears as a soft tissue structure of uniformly homogenous attenuation and is typically difficult to distinguish from surrounding muscles, vessels, and perineal structures (Wefer, 272). Therefore, for intraprostatic cancer(s), CT offers little usefulness in terms of screening, localization, and or subsequent biopsy guidance. As such, CT's primary role is in assessing prostate volume and detecting nodal metastases.

With regards to MRI, the depiction of the zonal anatomy of the prostate largely depends on the MRI pulse sequence used. For example, on T1-weighted images, the prostate demonstrates uniform signal intensity and the zonal anatomy is undifferentiated while on T2-weighted images, the zonal 
anatomy if well defined (Wefer, 272). In terms of prostate cancer screening, localization, and biopsy guidance there is no clear consensus in the literature. The efficacy of MRI to be used in such a capacity depends heavily on the MRI modality and protocol employed, the subsequent data analysis, and the definition of what constitutes a clinically significant cancerous lesion. In the literature, the sensitivity for prostate cancer(s) detection using a T2-weighted sequence ranges from 37 percent using a body coil (Quint, 323-327) to 96 percent using an endorectal coil (Hricak, 703-709). However, in both studies the specificities must also be considered; 100 percent and 36 percent respectively. The use of MRI dynamic contrast as well as MRI spectroscopy has also been explored with sensitivities reaching 73.5 percent (81 percent specificity) (Jager, 645-652) and 76 percent (57 percent specificity) respectively (Wefer, 400-404). An excellent analysis of the aforementioned MRI studies as well as others can be found in the review by Kirkham et al. (Kirkham, 1163-1175). Due the variable efficacy of MRI in detecting prostate cancer(s) as well as cost, like CT, MRI's current primary role is assessing prostate volume and detecting nodal metastases and seminal vessel invasion.

\section{$\underline{5.4}$ Prostate Cancer and Nuclear Medical Imaging}

As discussed above, conventional early detection tests and associated morphological imaging modalities are insufficient in confirming the presence or absence of intraprostatic cancer(s), localizing intraprostatic cancer(s), guiding biopsy, and or aiding in treatment decisions. Nuclear medical imaging, including PET, attempts to address these shortcomings, however the use of nuclear based imagers with regards to prostate cancer is an evolving landscape.

\subsubsection{Radiopharmaceuticals}

Currently, based on observations of increased glucose metabolism in cancer cells as compared to healthy cells by Warburg (Warburg, 519-530), ${ }^{18} \mathrm{~F}$-fluorodeoxy-glucose (FDG) is the most commonly used radiotracer in PET oncology (Inoue, 1-9). FDG has proven effective in imaging, staging, and monitoring varies cancers including lung cancer (Sazon, 417-421), head and neck cancer (Adams, 12551260), colorectal cancer (Hung, 1375-1378), and breast cancer (Scheidhauer, 618-623), however, the same can not be said for prostate cancer. Imaging prostate cancer(s) with FDG is problematic for two reasons. First, the uptake of FDG by prostate caner(s) is low due to the slow growth of a majority of prostate carcinomas. In a study of 64 patients (48 with untreated prostate caner and 16 with confirmed $\mathrm{BPH}$ ) by Effert et al. low FDG uptake was observed in 81 percent of primary prostate tumors (Effert, 994-998). A separate study by Hofer et al. (Hofer, 31-35) confirms the low uptake of FDG by prostate carcinomas. In both studies, it was concluded that due to the low uptake of FDG by prostate carcinomas there was either significant overlap or no difference in FDG uptake between BPH tissue, prostatitis, postoperative scar tissue, and cancerous prostatic tissue. As a result, prostate cancer differentiation was extremely difficult. Second, FDG is rapidly expelled in the urine, with areas of "high" uptake within the prostate concealed by an overwhelming abundance of activity in the ureters and bladder (Hara, 990-995). Therefore, taking into account both factors, the use of FDG to visualize as well as localize intraprostatic cancer(s) is extremely limited at best.

In 1998, in an effort so circumvent the challenges associated with FDG and clearly differentiate and localize intraprostatic cancer(s) as well as local metastasis Hara et al. proposed the use of ${ }^{11} \mathrm{C}$-choline (Hara, 990-995). In a ten patent study Hara et al. demonstrated the use of ${ }^{11} \mathrm{C}$-choline to successful detect the primary prostatic malignancy (histological verified prior via biopsy) in each patient with the prostate being the only organ in the pelvic to significantly uptake ${ }^{11} \mathrm{C}$-choline and negligible urinary and or bladder activity observed. Unlike FDG uptake which depends on the rate of glucose metabolism within cancer cells, the preferential and marked uptake of ${ }^{11} \mathrm{C}$-choline observed in the prostate is a function of increased phospholipid metabolism. Abnormalities in phospholipid metabolism are a hallmark of caner 
cells (Podo, 413-439) and neoplastic tissue with prior studies by Hara and colleagues (Hara, 842-847; Shinoura, 497-503) demonstrating increased accumulation of ${ }^{11} \mathrm{C}$-choline in neoplastic tissue as compared to healthy tissue. Choline is vital component of phopholipids in the cell membrane, thus increased phospholipid metabolism and synthesis results in increased ${ }^{11} \mathrm{C}$-choline uptake. Other studies by Picchio et al. and Kotzerke et al. (Picchio, 1337-1340; Kotzerke, 1415-1419) corroborate Harra's 1998 results with work by Yamaguchi et al. suggesting ${ }^{11} \mathrm{C}$-choline PET may provide increased accuracy for primary prostate cancer localization compared to MRI/MRS (Yamaguchi 742-748).

In term of ${ }^{11} \mathrm{C}$-choline specificity, studies by Reske et al., Scher et al., and de Jong et al. not only demonstrate a high uptake of ${ }^{11} \mathrm{C}$-choline in the prostate but a significantly higher uptake of ${ }^{11} \mathrm{C}$-choline in prostate cancer(s) as compared to BPH and prostatitis in an overwhelming majority of patients (Reske, 1249-1254; Scher, 45-53; de Jong 18-23). This differential uptake results in a clear delineation between prostate cancer(s) and surrounds benign tissue. In the study by Scher et al. 58 patents were examined. Of the 58, 37 had histologically verified prostate cancer with ${ }^{11} \mathrm{C}$-choline PET missing the primary malignancy in 5 individuals. In the remaining 21 patients who showed no signs of malignancy ${ }^{11} \mathrm{C}$ choline PET was a false positive in 8 individuals. The false positives were attributed to uptake by BPH and or prostatitis with only 2 individuals showing neither benign ailment. It should be noted that while

${ }^{11} \mathrm{C}$-choline exhibits fare less uptake within the bladder and ureters than FDG, in all three of the above mentioned studies moderate to significant bladder activity, of which did interfere with prostate imaging, was observed in ratios of 9:26, 19:58, and 3:30 respectively.

Along with ${ }^{11} \mathrm{C}$-choline there are other choline derivatives including ${ }^{11} \mathrm{C}$-acetate (Kato, 14921495), ${ }^{11} \mathrm{C}$-methionine (Tóth, 66-69), and ${ }^{18} \mathrm{~F}$-florocholine employed in a dual-phase protocol (Kwee, 262269) being investigated as applicable radiopharmaceuticals for prostate cancer visualization and localization.

\subsubsection{Prostate Specific Nuclear Medical Instrumentation}

In response to the inadequacies of current imaging modalities with regards to detection and localization of intraprostatic cancer(s) as well as the development of new radiopharmaceuticals capable of delineating prostate cancer(s) from surrounding tissue, there has been considerable interest in recent years in the development and optimization of novel nuclear medical instruments specific to prostate cancer imaging. When compared to clinical whole body PET scanners which are expensive, exhibit poor photon detection efficiency $(<1 \%$ ) and spatial resolution of 4-5 mm FWHM (Rohren, 305-306), as well as a large field of view (FOV), dedicated prostate imagers appear extremely attractive. Such dedicated imagers would be much smaller and thus substantial less expensive as well as mobile. They would also offer improved spatial resolution, photon detection efficiency, and a smaller field of view thus reducing image degrading background from the uptake of activity from surrounding organs. Monte Carlo simulation by Huh et al. suggest an APD and LSO scintillation crystal based endorectal probe operating in coincidence with a conventional clinical PET scanner can achieve $1 \mathrm{~mm}$ spatial resolution FWHM and increased photon detection efficiency (Huh, 339-343). While Monte Carlo simulations are encouraging, the development of functional and potentially clinically viable detectors is of greater interest. Currently, there are a handful of groups endeavoring in such a pursuit.

Work by Huber and colleagues centers around the development of prostate specific PET scanner based on two elliptical detector banks. The detector banks are situated on either side of a patient bed and operate in coincidence. The bank below the patient bed is fixed while the one above is telescopic to allow patient access. The prostate is centered within the field of view with each detector module angled towards the camera center (Huber, 1506-1511; Qi, 107-113). Initial phantom results demonstrate the detectors ability to resolve a cluster of four axially mounted line sources ( $5 \mathrm{~mm}$ separation between each 
line source) placed both 4 and $8 \mathrm{~cm}$ radially from the detectors center. In accordance with NEMA standards, spatial resolution was determined such that; the transverse spatial resolution $1 \mathrm{~cm}$ and $10 \mathrm{~cm}$ radially from the detector center was $4 \mathrm{~mm}$ FWHM and $5.5 \mathrm{~mm}$ FWHM respectively, and the tangential spatial resolution $10 \mathrm{~cm}$ radially from the detector center was $4 \mathrm{~mm}$ FWHM (Huber, 2653-2659). The use of two external coincidence detectors has also been explored by Turkington et al. utilizing two radially adjustable planer detectors (Turkington, 2806-2809) originally designed for a PET mammography system (Turkington, 10-16). The results were positive, however, image quality was not optimal.

In contrast, there are other groups which propose the use of an internal endorectal prostate probe. One such group is focusing on the development of a Compton scatter based imaging probe. Such a detector is based on the Compton imaging principal in which ionizing radiation incident on a scatter detector placed close to the source is Compton scattered and then absorbed in a second detector. The two detectors operate in coincidence, with the first scatter detector providing electronic collimation thus decoupling resolution and sensitivity. Position and energy measurements in both detectors allow for the determination of the ionizing radiation's origin within a conical ambiguity. A prototype external Compton probe has been built and tested (Bernabeu, 58-61; Llosá, 4168-4171, Lacasta, 3032-3035) with the most resent results reported by Llosá et al. Llosá reports a maximum resolution of $5 \mathrm{~mm}$ FWHM for a $356 \mathrm{keV}$ photon and a source placed approximately $11 \mathrm{~cm}$ from the scatter detector (Llosá, 936-941). While this result is a marked improvement over SPECT, it offers little advantage over clinical whole body PET scanners currently offered. Of greater concern is the fact that the prototype, while a prototype, is garishly unfeasible as a prostate probe in terms of size and the ability to achieve comparable, if not better, resolution with a clinically viable probe is unknown.

Two additional examples of endorectal probes being developed and tested include work by Cui et al., as well as, Levin and Vandenbroucke et al. First, studies by Cui focus on the development of a Cadmium Zinc Telluride (CZT) based gamma camera. The gamma camera is constructed from two $5 \mathrm{~mm}$ thick 6 x 8 element pixelated CZT arrays (pixel pitch of $2.46 \mathrm{~mm}$ ) coupled side by side to an applicationspecific-integrated-circuit (ASIC) all of which is housed in a stainless steel sheath with an outer diameter of $25 \mathrm{~mm}$. Using Tc-99m, energy resolution was shown to be 5\% FWHM at $140 \mathrm{keV}$ (a significant improvement over commonly used scintillation detectors with typical energy resolution ranging from $15 \%$ to $30 \%$ FWHM) and a spatial resolution of $5.7 \mathrm{~mm}$ was obtained when the probe was placed $23 \mathrm{~mm}$ from the source (the approximate distance between the endorectal inserted probe and the center of the prostate) (Cui, 1-8). While the spatial resolution is on par with clinical whole body PET systems currently available, these initial results are encouraging, especially when considering the practical, and potentially clinically viable, size of the probe.

Second, studies by Levin and Vandenbroucke et al. focus on the development of a novel compact endorectal PET probe operating in coincidence with an external panel detector. The probe is constructed from two separate detector modules of which are mounted one in front of the other on a common flex circuit. Each module is comprised an array of scintillation crystals which is coupled to a thin PSAPD. In an effort to maximize light collection efficiency, the crystals are oriented such that the long side of each crystal is coupled to the PSAPD. Ultimately it is proposed the probe consist of sixteen total detector modules, stacked atop one another in an $8 \times 2$ configuration. Initial results for one module utilizing a $3 \mathrm{x}$ 8 array of $1 \times 1 \times 3 \mathrm{~mm}^{3}$ LSO scintillation crystals (half ground and half polished) and no intracrystal reflector resulted in energy resolution ranging from $9.7 \%$ to $14.1 \%$ at $511 \mathrm{keV}$, average $\mathrm{x}-\mathrm{y}$ spatial resolution of $1.1 \mathrm{~mm}$ FWHM, and, unlike any of the aforementioned prostate specific imagers, an average depth of interaction resolution ranging from $2.97 \pm 0.07 \mathrm{~mm}$ FWHM to $3.03 \pm 0.05 \mathrm{~mm}$ FWHM (Levin). The most recent results, as reported by Vandenbroucke et al., demonstrate that for one layer utilizing two $8 \times 8$ arrays of $0.91 \times 0.91 \times 1 \mathrm{~mm}^{3}$ optically isolated LYSO scintillation crystals, average energy resolution for "face on" and "edge on" incident radiation was 13.5\% $\pm 0.7 \%$ FWHM and 14.6\% $\pm 1.7 \%$ 
FWHM respectively. Average x-y spatial resolution, when measured in the "edge on" configuration, was $.837 \pm 0.045 \mathrm{~mm}$ FWHM (Vandenbroucke, 5895-5911). DOI resolution was not explicitly quoted, however, as DOI resolution is a function of the lateral length of the scintillation crystal of interaction and nearly cubic scintillation crystals were used, spatial x-y resolution and depth resolution should be similar. While these results are impressive, there are serious questions concerning performance, construction viability, and increased cost when more than one layer is attempted.

\subsubsection{Dual Modality PET/TRUS}

Along with the development of prostate specific nuclear medical instruments there is growing interest in the fusion of PET and TRUS. The co-registration of both modalities would provide a structural anatomic view of the pelvic region and the prostate (TRUS) as well as functional metabolic information used to localize suspected cancerous lesions (PET). Advantages of a co-registration between PET and TRUS include improved biopsy guidance and increased rate of detection of prostate cancer recurrence. Currently there have been no clinical studies using PET/TRUS co-registration, however phantom studies by Huber et al. are promising (Huber, 2187-2190; Huber, 674-681).

\subsection{Overview}

Approximately 1 in 6 men will be diagnosed with prostate cancer during their lifetime (ACS). While prostate cancer is typically slow growing and a majority of men will die with rather than of prostate cancer, the current methods for screening and diagnosis prostate cancer are archaic. The PSA blood tests are unreliability and current imaging modalities employed (US, CT, MRI) are unable to localize intraprostatic cancer(s) thus making core need biopsy truly a "shot in the dark" at best. Nuclear medical instruments dedicated for prostate imaging seek to address these issues. Currently, in response to new radiopharmaceuticals capable of delineating prostate cancer(s) from surrounding tissue, there are a handful of groups exploring both external and internal prostate specific imagers. The work and studies presented in the forthcoming chapter will focus on the optimization and performance of an endorectal prostate specific depth of interaction based PET detector operating in coincidence with an external panel detector developed at West Virginia University.

\section{References}

Adams, S., R. P. Baum, T. Stuckensen, K. Bitter, and G. Hör, "Prospective comparison of ${ }^{18}$ F-FDG PET with conventional imaging modalities (CT, MRI, US) in lymph node staging of head and neck cancer.” European Journal of Nuclear Medicine and Molecular Imaging, vol. 25, issue 9, pp. 1255-1260, 1998.

Algaba, F., I. Trias, and Y. Arce, “Natural History of Prostatic Carcinoma: The Pathologist’s Perspective.” Prostate Cancer. eds. J. Ramon and L. Denis, series eds. P. M. Schlang and H. J. Senn, vol. 175, Berlin: Springer, 2007, pp. 9-24, Print. Recent Results in Cancer Research

American Cancer Society, Prostate Cancer, [Online] Available: http://www.cancer.org/Cancer/ProstateCancer/DetailedGuide/index , Atlanta, GA, 2012.

Aumüller, G., and J. Seitz, "Protein Secretion and Secretory Processes in Male Accessory Sex Glands." International Review of Cytology, vol. 121, pp. 127-231, 1990.

Bassett, M. T., L. M. Levy, C. Chetsanga, and E. Chokunonga, "Zimbabwe National Cancer Registry: summary data 1986-1989. National Cancer Registry Advisoty Committee.” Central African Journal of Medicine, vol. 38, issue 3, pp. 91-94, 1992. 
Bernabeu, J., N. H. Clinthorne, Y. Dewaraja, C. Lacasta, G. Llosá, M. Mikuž, S. Roe, W. L. Rogers, A. Studen, P. Weilhammer, L. Zhang, and D. Žontar, "Development of a high efficiency and high resolution Compton probe for prostate imaging.” Nuclear Instruments and Methods in Physics Research A, vol. 527, issues 1-2, pp. 58-61, 2004.

Bostwich, D. G. "High Grad Prostatic Intraepithelial Neoplasia: The Most Likely Precursor to Prostate Cancer.” Cancer, vol. 75, issue S7, pp. 1823-1836, 1995.

Breslow, N., C. W. Chan, G. Dhom, R. A. B. Drury, L. M. Franks, B. Gellei, Y. S. Lee, S. Lundberg, B. Sparke, N. H. Sternbya, and H. Tulinius, "Latent carcinoma of prostate at autopsy in seven areas. Collaborative study organized by the International Agency for Research on Cancer Lyons, France.” International Journal of Cancer, vol. 20, issue 5, pp. 680-688, 1977.

Bullock, A. D., and G. L. Andriole, "Screening for Prostate Cancer: Prostate-Specific Antigen, Digital Rectal Examination, and Free, Density, and Age-Specific Derivatives.” Prostate Cancer: Principles \& Practice. eds. P. W. Kantoff, P. R. Carroll, and A. V. D’Amico, $2^{\text {nd }}$ eds. R. K. Ross, J. T. Isaacs, and H. I. Scher, Philadelphia: Lippincott Williams \& Wilkins, 2002.

Carter, H. B., U. M. Hamper, S. Sheth, R. C. Sanders, J. I. Epstein, P. C. Walsh, "Evaluation of transrectal ultrasound in the early detection of prostate cancer.” Journal of Urology, vol. 142, issue 4, pp. 1008-1010, 1989.

Chou, Roger, J. M. Croswell, T. Dana, C. Bougatsos, I. Blazina, R. Fu, K. Gleitsmann, H. C. Koenig, C. Lam, A. Maltz, B. Rugge, and K. Lin, "Screening for Prostate Cancer: A Review of the Evidence for the U. S. Preventive Service Task Force.” Annals of Internal Medicine, vol. 155, issue 11, pp. 762-771, 2011.

Cui, Y., T. Lall, B. Tsui, J. Yu, G. Mahler, A. Bolotnikov, P. Vaska, G. De Geronimo, P. O’Connor, G. Meinken, J. Joyal, J. Barrett, G. Camarda, A. Hossain, K. H. Kim, G. Yang, M. Pomper, S. Cho, K. Weisman, Y. Seo, J. Babich, N. LaFrance, and R. B. James, “Compact CdZnTe-based gamma camera for prostate cancer imaging.” Presented at the 2011 IEEE Nuclear Science Symposium and Medical Imaging Conference, Valencia, Spain, pp. 1-8, October, 2011.

de Jong, I. J., J. Pruim, P. H. Elsinga, W. Vaalburg, H. J. A. Mensink, "Visualization of Prostate Cancer with ${ }^{11}$ CCholine Positron Emission Tomography.” European Urology, vol. 42, issue 1, pp. 18-23, 2002.

De Marzo, A. M., V. L. Marchi, J. I. Epstein, and W. G. Nelson, "Proliferative Inflammatory Atrophy and the Prostate: Implications for Prostatic Carcinogenesis.” American Journal of Pathology, vol. 155, issue 6, pp. 1985-1992, 1999.

Effert, P. J., R. Bares, S. Handt, J. M. Wolff, U. Büll, and G. Jakse, "Metobolic Imaging of Untreated Prostate Cancer by Positron Emission Tomography with ${ }^{18}$ Fluorine-labled Deoxyglucose.” Journal of Urology, vol. 155, issue 3, pp. 994-998, 1996.

Ferlay J., H. R. Shin, F. Bray, D. Forman, C. Mathers, and D. M. Parkin. GLOBOCAN 2008 v1.2, Cancer Incidence and Mortality Worldwide: IARC CancerBase No. 10 [Internet]. Lyon, France: International Agency for Research on Cancer; 2010. Available from: http://globocan.iarc.fr, accessed on 14/January/2012.

Gleason, D. F., “The Veteran’s Administration Cooperative Urologic Research Group: histologic grading and clinical staging of prostate carcinoma.” Urologic Pathology: The Prostate, Philadelphia: Lea and Febiger, 1977.

Guileyardo, J. M., W. D. Johnson, R. A. Welsh, K. Akazaki, and P. Correa, "Prevalence of latent prostate carcinoma in two U.S. populations.” Journal of the National Cancer Institute, vol. 65, issue 2, pp. 311-316, 1980.

Hara, T., N. Kosaka, and H. Kishi, “PET Imaging of Prostate Cancer Using Carbon-11-Choline.” Journal of Nuclear Medicine, vol. 39, issue 6, pp. 990-995, 1998. 
Hara, T., N. Kosaka, N. Shinoura, T. Kondo, "PET imaging of brain tumor with [methyl- ${ }^{11} \mathrm{C}$ ] choline.” Journal of Nuclear Medicine, vol. 38, issue 6, pp. 842-847, 1997.

Hodge, K. K., J. E. McNeal, and T. A. Stamey, “ Ultrasound guided transrectal core biopsy of the palpably abnormal prostate.” Journal of Urology, vol. 142, issue 1, pp. 66-70, 1989.

Hofer, C., C. Laubencacher, T. Block, J. Breul, R. Hartung, and M. Schwaiger, "Flourine-18-Fluorodeoxyglucose Positron Emission Tomography is Useless for the Detection of Local Recurrence after Radical Prostatectomy.” European Urology, vol. 36, issue 1, pp. 31-35, 1999.

Hricak, H., S. White, D. Vigneron, J. Kurhanewicz, A. Kosco, D. Levin, J. Weiss, P. Narayan, and P. R. Carroll, "Carcinoma of the prostate gland: MR imaging with pelvic phase-array coils versus integrated endorectal-pelvic phase-array coils.” Radiology, vol. 193, issue 3, pp. 703-709, 1994.

Huber, J. S., Q. Peng, W. W. Moses, B. W. Reutter, J. Pouliot, and I. C. Hsu, "Development of a PET-Transrectal Ultrasound Prostate Imaging System.” IEEE Transactions on Nuclear Science, vol. 58, issue 3, pp. 674-681, 2001.

Huber, J. S., S. E. Derenzo, J. Qi, W. W. Moses, R. H. Huesman, and T. F. Budinger, “Conceptual Design of a Compact Positron Tomograph for Prostate Imaging.” IEEE Transactions on Nuclear Science, vol. 48, issue 4, pp. 1506-1511, 2001.

Huber, J. S., W. S. Choong, W. W. Moses, J. Qi, J. Hu, G. C. Wang, D. Wilson, S. Oh, R. H. Huesman, S. E. Derenzo, and T. F. Budinger, "Initial Results of a Positron Tomograph for Prostate Imaging.” IEEE Transactions on Nuclear Science, vol. 53, issue 5, pp. 2653-2659, 2006.

Huber, J. S., W. W. Moses, J. Pouliot, and I. C. Hsu, “Dual-modality PET/ultrasound imaging of the prostate.” IEEE Nuclear Science Symposium Conference Record, vol. 4, pp. 2187-2190, 2005.

Huh, S. S., N. H. Clinthorne, and W. L. Rogers, "Investigation of an internal PET probe for prostate imaging." Nuclear Instruments and Methods in Physics Research A, vol. 579, issue 1, pp. 339-343, 2007.

Hung, G. U., Y. C. Shiau, S. C. Tsai, T. H. Choa, Y. J. Ho, and C. H. Kao, "Value of ${ }^{18}$ F-flouro-deoxyglucose positron emission tomography in the evaluation of recurrent colorectal cancer." Anticancer Research, vol. 21, issue 2B, pp. 1375-1378, 2001.

Inoue, T., N. Oriuchi, K. Tomiyoshi, and K. Endo, "A shifting landscape: What will be next FDG in PET oncology.” Annals of Nuclear Medicine, vol. 16, issue 1, pp. 1-9, 2002.

Jager, G. J., E. T. Ruijter, A. A. van de Kaa, J. J. de la Rosette, R. O. Oosterhof, J. R. Thornbury, S. H. Ruijs, and J. O. Barentsz, "Dynamic TurboFLASH subtraction technique for contrast-enhanced MR imaging of the prostate: correlation with histopathologic results.” Radiology, vol. 203, issue 3, pp. 645-652, 1997.

Kato, T., E. Tsukamoto, Y. Kuge, T. Takei, T. Shiga, N. Shinohara, C. Katoh, K. Nakada, and N. Tamaki, "Accumulation of $\left[{ }^{11} \mathrm{C}\right]$ acetate in normal prostate and benign prostatic hyperplasia: a comparison with prostate cancer.” European Journal of Nuclear Medicine, vol. 29, issue 11, pp. 1492-1495, 2002.

Kirby, R. S., and Manish I. Patel, Fast Facts: Prostate Cancer. $6^{\text {th }}$ ed. Oxford: Health Press, 2009.

Kirkham, A. P. S., M. Emberton, and C. Allen, "How Good is MRI at Detecting and Characterizing Cancer within the Prostate?” European Urology, vol. 50, issue 6, pp. 1163-1175, 2006.

Kotzerke, J., J. Prang, B. Neumaier, B. Volkmer, A. Guhlmann, K. Kleinschmidt, R. Hautmann, and S. N. Reske, "Experience with carbon-11 choline positron emission tomography in prostate carcinoma." European Journal of Nuclear Medicine, vol. 27, issue 9, pp. 1415-1419, 2000. 
Kwee, S. A., H. Wei, I. Sesterhenn, D. Yun, and M. N. Coel, "Localization of Primary Prostate Cancer with DualPhase ${ }^{18}$ F-Flourocholine PET.” Journal of Nuclear Medicine, vol. 47, issue 2, pp. 262-269, 2006.

Lacasta C., J. Bernabeu, V. Borshchov, D. Burdette, E. Chesi, N. H. Clinthorne, Y. K. Dewaraja, K. Honscheid, H. Kagan, A. Listratenko, G. Llosá, M. Mikuž, P. Modesto, M. Protsenko, W. L. Rogers, V. Starkov, A. Studen, P. Weilhammer, L. Zhang, G. Zinovjev, D. Žontar, "Development and test of TAB bonded micro-cables for silicon detectors in a Compton prostate probe.” IEEE Nuclear Science Symposium Conference Record, vol. 5, pp. 3032-3035, 2005.

Levin, C., "New Photon Sensor Technologies for PET in Prostate-Specific Imaging Configurations.” Presented at the Topical Symposium on Advanced Molecular Imaging Techniques in the Detection, Diagnosis, Therapy, and Follow-Up of Prostate Cancer, Rome, Italy, December, 2005, [Online] Available: http://www.iss.infn.it/garibaldi/temp/Levin.pdf.

Lin, Kenneth, J. M. Croswell, H. Koenig, C. Lam, A. Maltz, "Prostate-Specific Antigen-Based Screening for Prostate Cancer: An Evidence Update for the U.S. Preventive Service Task Force.” [Online] Available: http://www.uspreventiveservicestaskforce.org/uspstf12/prostate/prcascres.pdf, Agency for Healthcare Research and Quality Publication No. 12-05160-EF-1, Rockville, MD, 2011.

Llosá, G., J. Bernabeu, D. Burdette, E. Chesi, N. H. Clinthorne, K. Honscheid, H. Kagan, C. Lacasta, M. Mikuž, P. Modesto, W. L. Rogers, A. Studen, and P. Weilhammer, "Last Results of a First Compton Probe Demonstrator.” IEEE Transactions on Nuclear Science, vol. 55, issue 3, pp. 936-941, 2008.

Llosá, G., J. Bernabeu, D. Burdette, E. Chesi, V. Cindro, N. H. Clinthorne, Y. K. Dewaraja, K. Honscheid, S. S. Huh, H. Kagan, C. Lacasta, N. Malakhov, M. Mikuž, P. Modesto, W. L. Rogers, J. Steinberg, A. Studen, P. Weilhammer, L. Zhang, and D. Žontar, "Development of a Pre-clinical Compton Probe Prototype for Prostate Imaging.” IEEE Nuclear Science Symposium Conference Record, vol. 7, pp. 4168-4171, 2004.

Main Line Health, “Anatomy of the Prostate.” [Online] Available: http://www.mainlinehealth.org/stw/Page.asp?PageID=STW038281, Philadelphia, PA, 2012.

McNeal, J. E., “Origin and evolution of benign prostatic enlargement.” Investigative Urology, vol. 15, issue 4, pp. 340-345, 1978.

McNeal, J. E., “The zonal anatomy of the prostate.” Prostate, vol. 2, issue 1, pp. 35-49, 1981.

Melchior, S. W., and M. K. Brawer, "Role of transrectal ultrasound and prostate biopsy.” Journal of Clinical Ultrasound, vol. 24, issue 8, pp. 463-471, 1996.

Myers, R. P. “Gross and Applied Anatomy of the Prostate.” Prostate Cancer: Principles \& Practice. eds. P. W. Kantoff, P. R. Carroll, and A. V. D’Amico, $2^{\text {nd }}$ eds. R. K. Ross, J. T. Isaacs, and H. I. Scher, Philadelphia: Lippincott Williams \& Wilkins, 2002.

National Cancer Institute, What You Need to Know About Prostate Cancer, [Online] Available: http://cancer.gov/cancertopics/wyntk/prostate, National Institute of Health Publication No. 08-1576, Bethesda, MD, 2012.

Picchio, M., C. Messa, C. Landoni, L. Gianolli, S. Sironi, M. Brioschi, M. Matarrese, D. V. Matei, F. de Cobelli, A. del Maschio, F. Rocco, P. Rigatti, and F. Fazio, "Value of $\left[{ }^{11} \mathrm{C}\right]$ Choline-Positron Emission Tomography for Re-staging Prostate Cancer: A Comparison with $\left[{ }^{18} \mathrm{~F}\right]$ Fluorodeoxygluucose-Positron Emission Tomography.” Journal of Urology, vol. 169, issue 4, pp. 1337-1340, 2003.

Podo, F. S. Canecari, R. Canese, M. E. Pisanu, A. Ricci, and E. Iorio, “Tumour Phospholipid Metabolism.” NMR in Biomedicine, vol. 12, issue 7, pp. 413-439, 1999. 
Qi, J., J. S. Huber, R. H. Huesman, W. W. Moses, S. E. Derenzo, and T. F. Budinger, "Septa Design for a Prostate Specific PET Camera.” IEEE Transactions on Nuclear Science, vol. 52, issue 1, pp. 107-113, 2005.

Quint, L. E., J. S. Van Erp, P. H. Bland, S. H. Mandell, E. A. Del Buono, H. B. Grossman, G. M. Glazer, and P. W. Gikas, "Carcinoma of the prostate; MR images obtained with body coil do not accurately reflect tumor volume.” American Journal of Roentgenology, vol. 156, issue 3, pp. 511-516, 1991.

Ravery, V., T. Billebaud, M. Toublanc, L. Boccon-Gibod, J. F. Hermieu, F. Moulinier, E. Blanc, V. Delmas, and L. Boccon-Bibod, “Diagnostic Value of Ten Systematic TRUS-Guided Prostate Biopsies.” European Urology, vol. 35, issue 4, pp. 298-303, 1999.

Reske, S. N., N. M. Blumstein, B. Neumaier, H. W. Gottfried, F. Finsterbusch, D. Kocot, P. Möller, G. Glatting, and S. Perner, "Imaging Prostate Cancer with ${ }^{11}$ C-Choline PET/CT.” Journal of Nuclear Medicine, vol. 47, issue 8, pp. 1249-1254, 2006.

Rodriguez, L. V., and M. K. Terris, "Risks and Complications for Transrectal Ultrasound Guided Prostate Needle Biopsy: A Prospective Study and Review of the Literature.” Journal of Urology, vol. 160, issue 6 part 1, pp. 2115-2120, 1998.

Rohren, E. M., T. G. Turkington, and R. E. Coleman, “Clinical Applications of PET in Oncology.” Radiology, vol. 231, issue 2, pp. 305-332, 2004.

Sakr, W. A., G. P. Haas, B. F. Cassin, J. E. Pontes, and J. D. Crissman, "The frequency of carcinoma and intraepithelial neoplasia of the prostate in young male patients.” Journal of Urology, vol. 150, issue 2 part 1, pp. 379-385, 1993.

Sazon, D. A., S. M. Santiago, G. W. Soo Hoo, A. Khonsary, C. Brown, M. Mandelkern, W. Blahd, and A. J. Williams, "Fluorodeoxyglucose-positron emission tomography in the detection and staging of lung cancer." American Journal of Respiratory and Critical Care Medicine, vol. 153, issue 1, pp. 417-421, 1996.

Scheidhauer, K., A. Scharl, U. Pietrzyk, R. Wagner, U. J. Göhring, K. Schomäcker,, and H. Schicha, "Quantitative $\left[{ }^{18} \mathrm{~F}\right]$ FDG positron emission tomography in primary breast cancer: clinical relevance and practicability.” European Journal of Nuclear Medicine and Molecular Imaging, vol. 23, issue 6, pp. 618-623, 1996.

Scher, B., M. Seitz, W. Albinger, R. Tiling, M. Scherr, H. C. Becker, M. Souvatzogluou, F. J. Gildehaus, H. J. Wester, and S. Dresel, "Value of ${ }^{11} \mathrm{C}$-choline PET and PET/CT in patients with suspected prostate cancer." European Journal of Nuclear Medicine and Molecular Imaging, vol. 34, issue 1, pp. 45-53, 2007.

Scherr, D. S., J. Eastham, M. Ohori, and P. T. Scardino, "Prostate biopsy techniques and indications: when, where, and how?” Seminars in Urologic Oncology, vol. 20, issue 1, pp. 18-31, 2002.

Schinoura, N., M. Nishijima, T. Hara, T. Haisa, H. Yamamoto, K. Fujii, I. Mitsui, N. Kosaka, T. Kondo, and T. Hara, "Brain tumors: detection with C-11 choline PET.” Radiology, vol. 202, issue 2, pp. 497-503, 1997.

Schröder, F. H., J. Hugosson, M. J. Roobol, T. L. J. Tammela, S. Ciatto, et al. "Screening and Prostate -Cancer Mortality in a Randomized European Study.” New England Journal of Medicine vol. 360, issue 13, pp. 13201328, 2009.

Shah, R. B., and Ming Zhou, Prostate Biopsy Interpretation: An Illustrated Guide. Berlin: Springer, 2011.

Taylor, L. S., D. J. Rubens, B. C. Porter, Z. Wu, P. A. di Sant'Agnese, G. Nadasdy, D. Pasternack, E. M. Messing, P. Nigwekar, and K. J. Parker, "Prostate Cancer: Three Dimensional Sonoelastography for in Vitro Detection." Radiology, vol. 237, issue 3, pp. 981-985, 2005.

Tóth, G., Z. Lengyel, L. Balkay, M. A. Salah, L. Trón, and C. Tóth, "Detection of Prostate Cancer with ${ }^{11}$ CMethionine Positron Emission Tomography.” Journal of Urology, vol. 173, issue 1, pp. 66-69, 2005. 
Turkington, T. G., M. F. Smith, T. C. Hawk, S. Majewski, B. J. Kross, R. Wojcik, A. G. Weisenberger, T. R. DeGrado, and R. E. Coleman, "PET Prostate Imaging with Small Planar Detectors.” IEEE Nuclear Science Symposium Conference Record, vol. 5, pp. 2806-2809, 2004.

Turkington, T. G., S. Majewski, A. G. Weisenberger, V. Popov, M. F. Smith, W. H. Sampson, R. Wojcik, and D. Kieper, "A large field of view positron emission mammography imager.” IEEE Nuclear Science Symposium Conference Record, vol. 3, pp. 1883-1886, 2002.

U.S. Preventive Service Task Force, "Screening for Prostate Cancer.” [Online] Available: http://www.uspreventiveservicestaskforce.org/uspstf/uspsprca.htm\#comment , Rockville, MD, 2011.

Vandenbroucke, A., A. M. K. Foudray, P. D. Olcott, and C. S. Levin, "Performance characterization of a new high resolution PET scintillation detector.” Physics in Medicine and Biology, vol. 55, issue 19, pp. 5895-5911, 2010.

Warburgh, O., F. Wind, and E. Negelein, “The Metabolism of Tumors in the Body.” Journal of General Physiology, vol. 8, issue 6, pp. 519-530, 1927.

Wefer, A. E., and H. Hricak, “Imaging and Staging of Prostate Cancer.” Prostate Cancer: Principles \& Practice. eds. P. W. Kantoff, P. R. Carroll, and A. V. D’Amico, $2^{\text {nd }}$ eds. R. K. Ross, J. T. Isaacs, and H. I. Scher, Philadelphia: Lippincott Williams \& Wilkins, 2002.

Wefer, A. E., H. Hricak, D. B. Vigneron, F. V. Coakley, Y. Lu, J. Wefer, U. Mueller-Lisse, P. R. Carroll, and J. Kurhanewicz, "Sextant Localization of Prostate Cancer: Comparison of Sextant Biopsy, Magnetic Resonance Imaging and Magnetic Resonance Spectroscopic Imaging with Step Section Histology.” Journal of Urology, vol. 164, issue 2, pp. 400-404, 2000.

Yamaguchi, T., J. Lee, H. Uemura, T. Sasaki, N. Takahashi, T. Oka, K. Shizukuishi, H. Endou, Y. Kubota, T. Inoue, "Prostate cancer: a comparative study of ${ }^{11} \mathrm{C}$-choline PET and MR imagine combine with proton MR spectroscopy.” European Journal of Nuclear Medicine and Molecular Imaging, vol. 32, issue 7, pp. 742-748, 2005. 


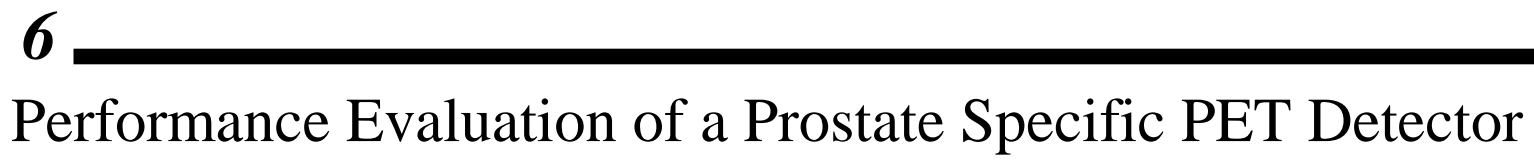

\subsection{Introduction}

Prostate cancer is not only the second most common form of cancer found in American men but also the second most lethal form of cancer (American Cancer Society (ACS)). Unfortunately, early detection methods employed to screen individuals are unreliable and current imaging modalities including US, CT, MRI are inadequate with regards to localizing and differentiating intraprostatic lesions. With the advent of new prostate specific radiopharmaceuticals such as various ${ }^{11} \mathrm{C}$ derivatives, including ${ }^{11} \mathrm{C}$-choline, which has been successfully employed as a PET molecular tracer for intraprostatic lesions detection (Hara, 990-995), there has been considerable interest in recent years in reevaluating nuclear medical imaging's role with regards to prostate cancer. However, conventional PET scanners with their large ring geometry are not only expensive but exhibit spatial resolution on the order of 4-5 mm FWHM (Rohren, 305-306) which has proven to be inefficient in the detection of intraprostatic lesions, small pelvic lymph node metastases, and the local invasions to nearby tissue (Oehr, 259-264). Consequently, small prostate specific nuclear medical instruments offering increased resolution, high photon detection efficiencies, and reduced cost are becoming extremely attractive. Therefore, the objective of this work is the development and testing of a compact depth of interaction based PET detector for prostate imaging. The detector is designed such that in subsequent studies the module can be packaged and employed as an endorectally insertable probe operating in coincidence with an external gamma detector or conventional PET scanner. Such a geometry would provide a high resolution image used to facilitate the reliable detection, localization, and characterization of small cancerous lesions within the prostate. Consequently, this increased performance will not only aid in diagnosis and biopsy guidance, but treatment planning as well.

The development and testing of the DOI based PET detector can be broken down into four separate studies: SensL SiPM based proof of concept studies, systematic Hamamatsu SiPM based pilot studies as well as full scale studies, and DOI based image reconstruction studies.

\subsection{SensL Proof of Concept Studies}

Proof of concept DOI studies were performed utilizing an apparatus similar to the one discussed in section 4.3. While similar, differences included a DOI module based on SensL SiPMs (SPMArray2), a 12 x 12 element scintillation array comprised of saw cut $1.0 \mathrm{~mm}$ x $1.0 \mathrm{~mm}$ x $10 \mathrm{~mm}$ pixels, and $2 \mathrm{~mm}$ thick light spreader windows (Fig. 6.1). Also, flexible printed circuit (FPC) cables were use to interface each SiPM to custom 16 channel differential pre-amplifiers connected to interface/power supply board (SPMArray2-A0 and SMPArray2A1). The second coincidence detector consisted of a $1 \mathrm{~cm}$ diameter Hamamatsu R1635 PMT optically coupled to a $1 \mathrm{~cm}^{3}$ LYSO scintillator. Separation distance between the DOI module and the coincidence detector was approximately $15 \mathrm{~cm}$.

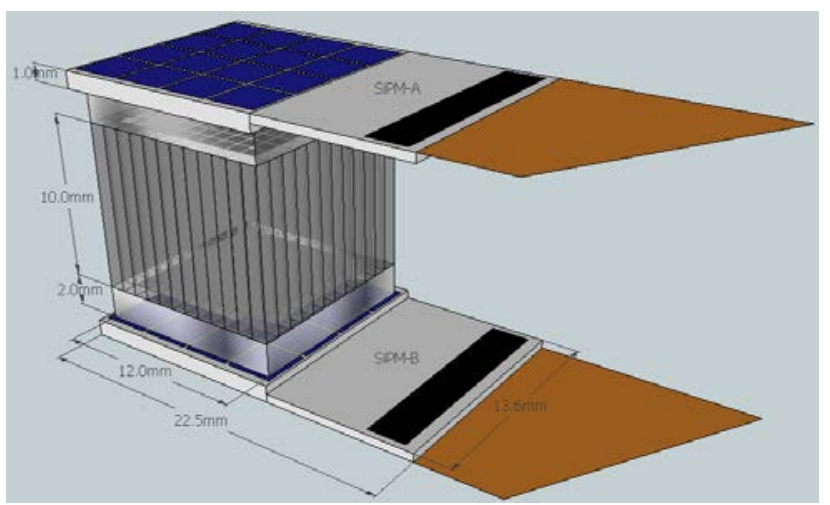

Fig 6.1 Schematic diagram of the sensL based DOI detector module. Dimensions of the module are approximately $22.5 \mathrm{~mm}$ x $13.6 \mathrm{~mm}$ x $16 \mathrm{~mm}$. 


\subsubsection{Methods}

Prior to any DOI studies being performed, a preliminary energy calibration followed by a mutual gain equalization calibration were preformed on the DOI module in order to ensure accurate DOI estimates in subsequent DOI studies. The energy calibration was preformed by first disabling the coincidence detector, specifying the coincidence circuitry to operate in singles mode, and irradiating the DOI module with a broad uniform beam from 4 equally spaced ${ }^{22} \mathrm{Na}$ button sources (Fig. 6.2b). Next, using the acquired flood histograms for each SiPM (Fig. 6.2c), crystal maps for each SiPM were created and saved in Kmax. Finally, with the crystal maps saved, the DOI module was again irradiated with a broad uniform beam and the subsequent $511 \mathrm{keV}$ photopeak for each scintillation pixel was documented and stored in Kmax. Prior to performing the mutual gain equalization calibration, a single centrally located LYSO pixel on which subsequent DOI studies would be preformed was selected in an ROI (region of interest) and is indicated by the red arrow in Figure 6.2c. Once again the DOI module was irradiated with a broad uniform beam in order to determine the energy resolution and the relationship between the output signals from both SiPMs for the selected pixel.

Next, a mutual gain equalization calibration was preformed as accurate DOI estimates are SiPM gain dependent. If the gain of both SiPMs are not equal DOI estimates can become skewed and DOI resolution degraded. The mutual gain equalization was preformed by first enabling the coincidence detector and placing a capillary tube $\left(0.5 \mathrm{~mm}\right.$ inner diameter) filled with a solution of ${ }^{18} \mathrm{~F}$ between both detector modules ( $1 \mathrm{~mm}$ from the DOI module) such that a narrow electronically collimated $511 \mathrm{keV}$ fan beam was incident on the center (5 mm in depth) of the DOI module scintillation array. Next, the reverse bias voltage of each SiPM was adjusted such that the centroid of the DOI ratio histogram for the single centrally selected pixel was positioned at 50\%. As a result of SiPM gain fluctuation with temperature, the gain of both SiPM was monitored throughout the following DOI studies with the reverse bias voltage of one or both SiPMs adjusted in order to maintain mutual gain equalization.

(a)

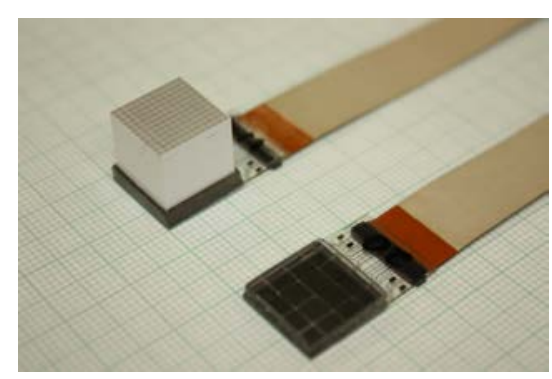

(b)

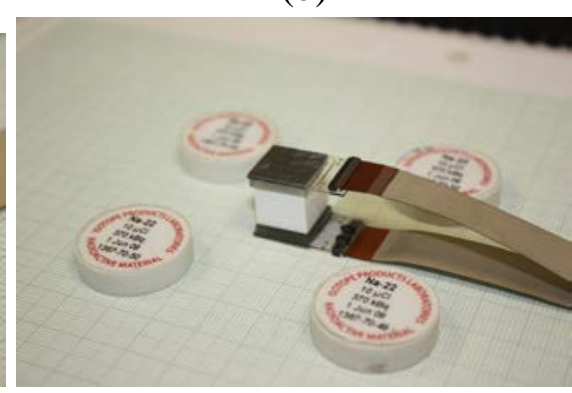

(c)

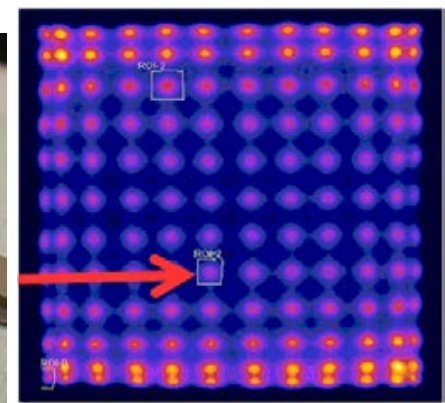

Fig. 6.2 (a) the SensL based DOI module being prepared for laboratory test (b) the SensL based DOI module with four equally spaced ${ }^{22} \mathrm{Na}$ sources, (c) flood histogram obtained from one SiPM of the DOI module when uniformly irradiated. All 144 LYSO pixels are visible and well differentiated. The single centrally located LYSO pixel selected for DOI studies is marked by the red arrow. Both photos (a) and (b) are against a $1 \mathrm{~mm}$ scale background.

Following both the energy and mutual gain equalization calibrations, DOI studies were preformed. Two separate trials were preformed. The first trial consisted of two capillary tubes spaced $5 \mathrm{~mm}$ apart and filled with a solution of ${ }^{18} \mathrm{~F}$. The capillary tubes were positioned between either detector module such that they were $1 \mathrm{~mm}$ from the DOI module, and irradiating the DOI scintillation array at depths of $3 \mathrm{~mm}$ and 8 $\mathrm{mm}$. The second trial was identical to the first, however three capillary tubes spaced $2.5 \mathrm{~mm}$ apart and positioned at irradiation depths of $2.5 \mathrm{~mm}, 5.0 \mathrm{~mm}$, and $7.5 \mathrm{~mm}$ were used. Fan beam width $\mathrm{r}$ incident on the DOI module scintillation array was approximated to be $0.571 \mathrm{~mm}$ using the following equation:

$$
r=s+\frac{b}{a}(s+d)
$$


with s equal to the source diameter, a equal to the distance between the source and the coincident module, $b$ equal to the distance between the source and the DOI module, and d equal to the side length of the coincidence detector.

\subsubsection{Results}

\section{Energy Characterization}

The energy spectra for the single centrally selected LYSO pixel are depicted in Fig. 6.3a-c. Upon applying a Gaussian + quadratic background fit to the sum energy spectra energy resolution averaged over all depths was shown to be approximately 20\%. The histogram of the signal from SiPM-A verses the signal from SiPM-B for the selected LYSO pixel is depicted in Fig. 6.3d. The bright crest corresponds to $511 \mathrm{keV}$ photopeak events and indicates an approximately linear relationship between signal A and signal B over all depths.

(a) SiPM-A

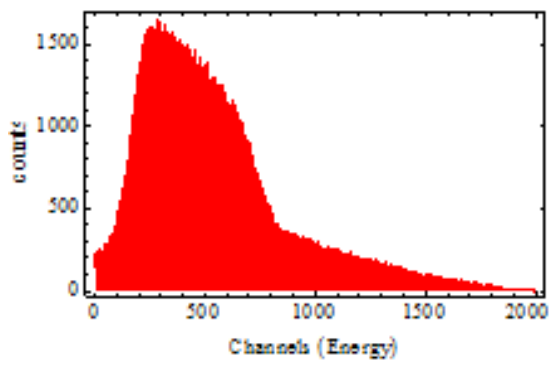

(d)

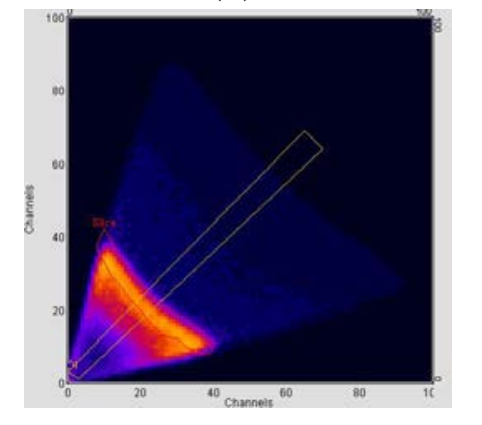

Extraction of DOI Information (b) SiPM-B

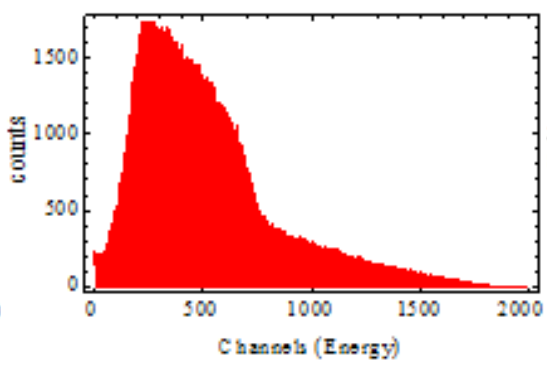

(c) Sum

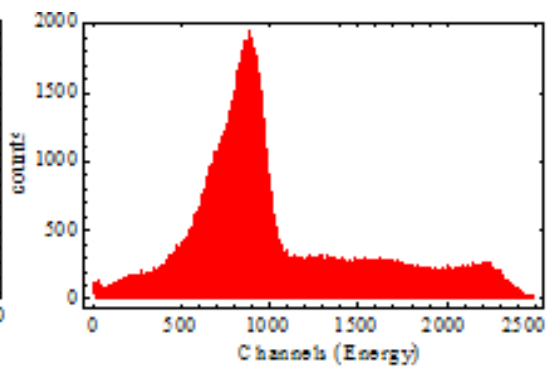

Fig. 6.3 (a) Energy spectra for SiPM-A, (b) energy spectra for SiPM-B, (c) sum energy spectra for both SiPM-A and B. The exponential decay in the energy spectra for each SiPM (a and b) is a function of the exponential decay in the magnitude of scintillation photons incident on each detector as the distance between each SiPM and the DOI is increased. The exponential light decay is a result of both the intracrystal reflector, as well as, the scintillation crystal surface finish and is the foundation for extraction DOI using the dual ended readout approach. (d) Signal from SiPM-A vs. SiPM-B for the uniformly irradiated LYSO pixel selected in Fig. 6.2c. The bright colored crest in (d) corresponds to $511 \mathrm{keV}$ photopeak events.

The DOI profile for the single selected LYSO pixel is shown in Fig. 6.4. Each peak was generated by applying a Gaussian + quadratic background fit to the binned DOI ratio data readout from Kmax. The solid red peaks correspond to the two capillary tubes at irradiating depths of $3 \mathrm{~mm}$ and $8 \mathrm{~mm}$ and the blue dashed peaks correspond to the three capillary tubes at irradiation depths of $2.5 \mathrm{~mm}, 5 \mathrm{~mm}$, and $7.5 \mathrm{~mm}$. While the FWHM of each peak in Fig. 6.4 corresponds to the DOI resolution at the particular depth irradiated, the resolution values are in units of DOI ratio bins and not standard units of length. The width (in millimeters) of each DOI ratio bin was determined by utilizing known ratios of distance in millimeters to distance in bins in order to calculated a pixel specific calibration factor k. For example, $2.5 \mathrm{~mm}$ in depth corresponds to the number of DOI ratio bins between zero and the centroid of the first peak in Fig. 6.4. Figure 6.5 shows the distance in DOI ratio bins between zero and the centroid of each successive peak plotted as a function of irradiation depth. As a result of the approximately linear relationship between signal-A and signal-B illustrated in Fig. 6.3d there is also an approximately linear relationship between DOI ratio and depth. Thus, a linear fit $\left(\mathrm{R}^{2} \rightarrow 0.9451\right)$ was applied to the points in Fig. 6.5. The 
equation of the linear fit takes the form of equation 4.1 with the slope of the linear fit equal to the mm/bin calibration factor k. Therefore, multiplying the FWHM of each peak by the calibration factor yields the DOI resolution in millimeters at each particular irradiation depth. DOI resolution for the single selected LYSO pixel averaged over all five irradiation depths was $1.27 \pm 0.06 \mathrm{~mm}$.

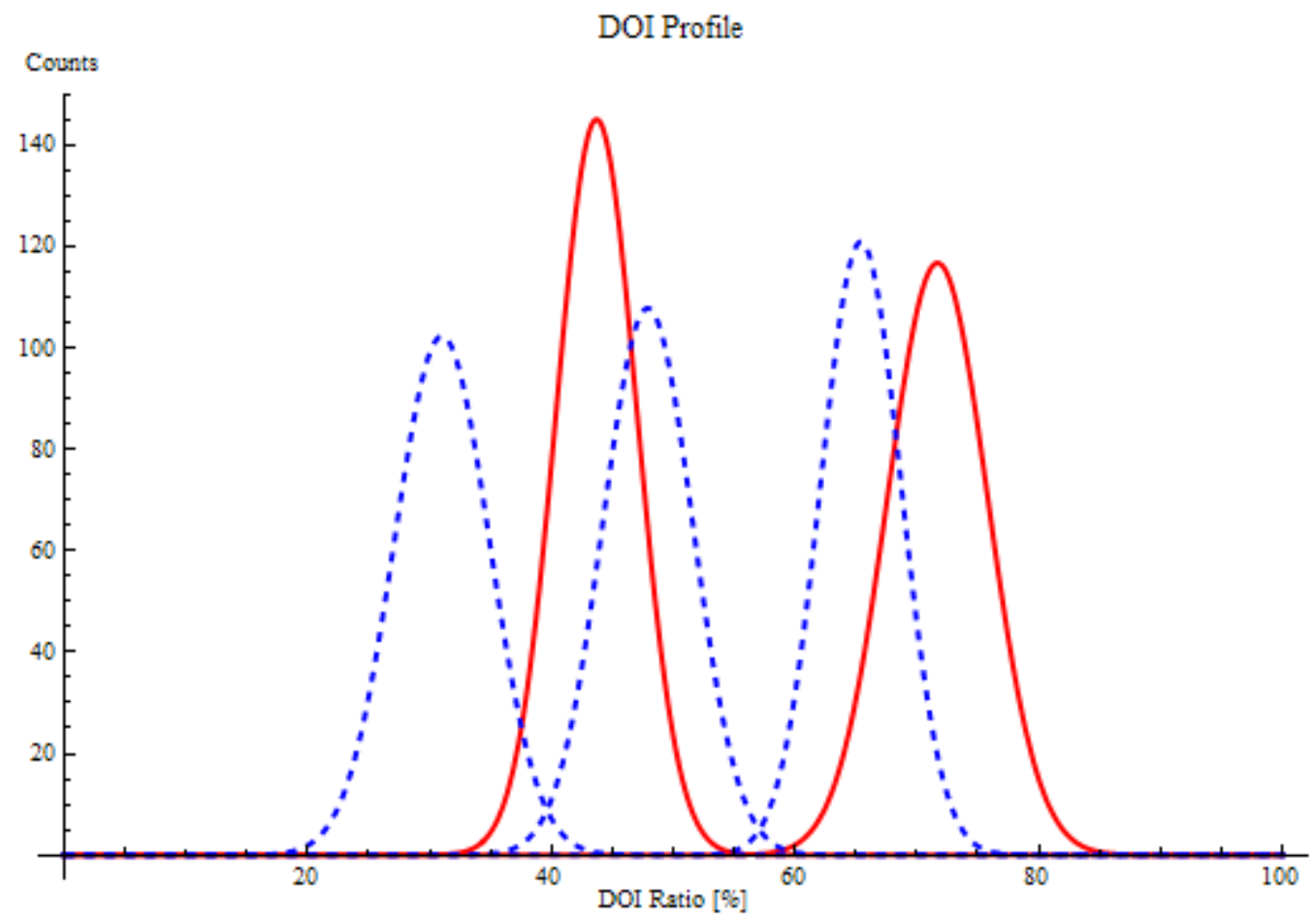

Fig. 6.4 DOI profile for the single centrally located LYSO pixel irradiated at five depths: $3 \mathrm{~mm}$ and $8 \mathrm{~mm}$ (red solid) and $2.5 \mathrm{~mm}, 5 \mathrm{~mm}$, and $7.5 \mathrm{~mm}$ (blue dashed).

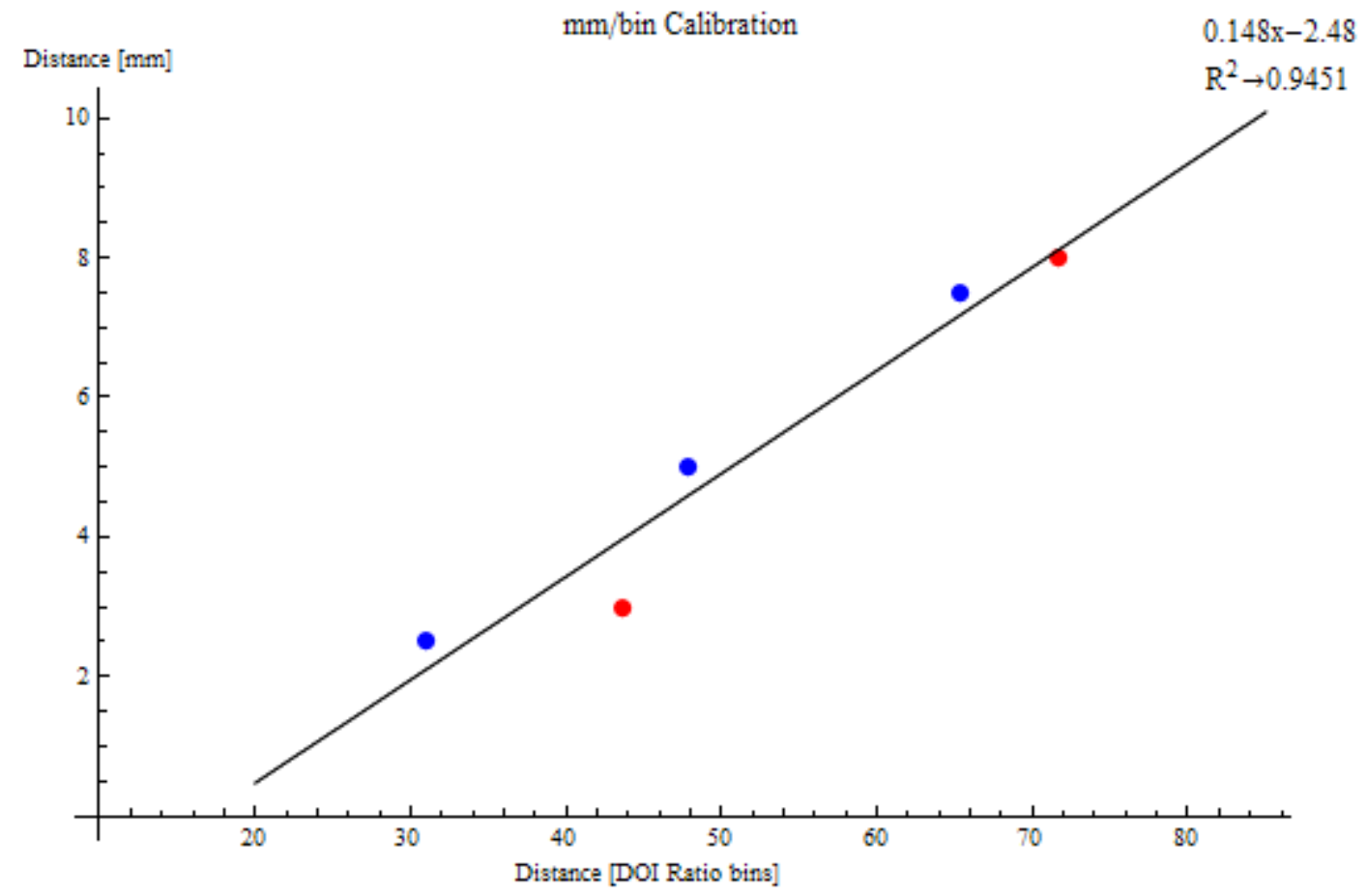

Fig. 6.5 Plot of the known mm/bin ratios from Fig. 6.4. A linear fit was applied with the slope of the fit equal to the $\mathrm{mm} /$ bin calibration factor $\mathrm{k}$. 


\section{$\underline{\text { 6.2.3 Discussion }}$}

Depth of interaction information is extracted on an event by event and pixel by pixel basis with the linear fit from Fig. 6.5 providing a pixel specific calibration function equating DOI ratio to a physical depth. However, the calibration function is only useful if it provides accurate estimates of the depth at which an event occurred. In order to determine the efficacy and accuracy of the calibration function from Fig. 6.5 the centroid values for each peak in Fig. 6.4 were substituted into the calibration equation with the resultant calculated depths of interaction plotted as a function of the true depths of interaction i.e. the irradiation depths at which each capillary tube was positioned (Fig. 6.6). The error bars in Fig. 6.6 are equal to the FWHM of each DOI response peak in Fig. 6.4. The slope and associated $\mathrm{R}^{2}$ value $(0.9451)$ of the linear fit in Fig. 6.6 indicates the accuracy of the calibration function. As both the slope and $\mathrm{R}^{2}$ values approach one, the discrepancy between the calculated depth and the true depth approaches zero.

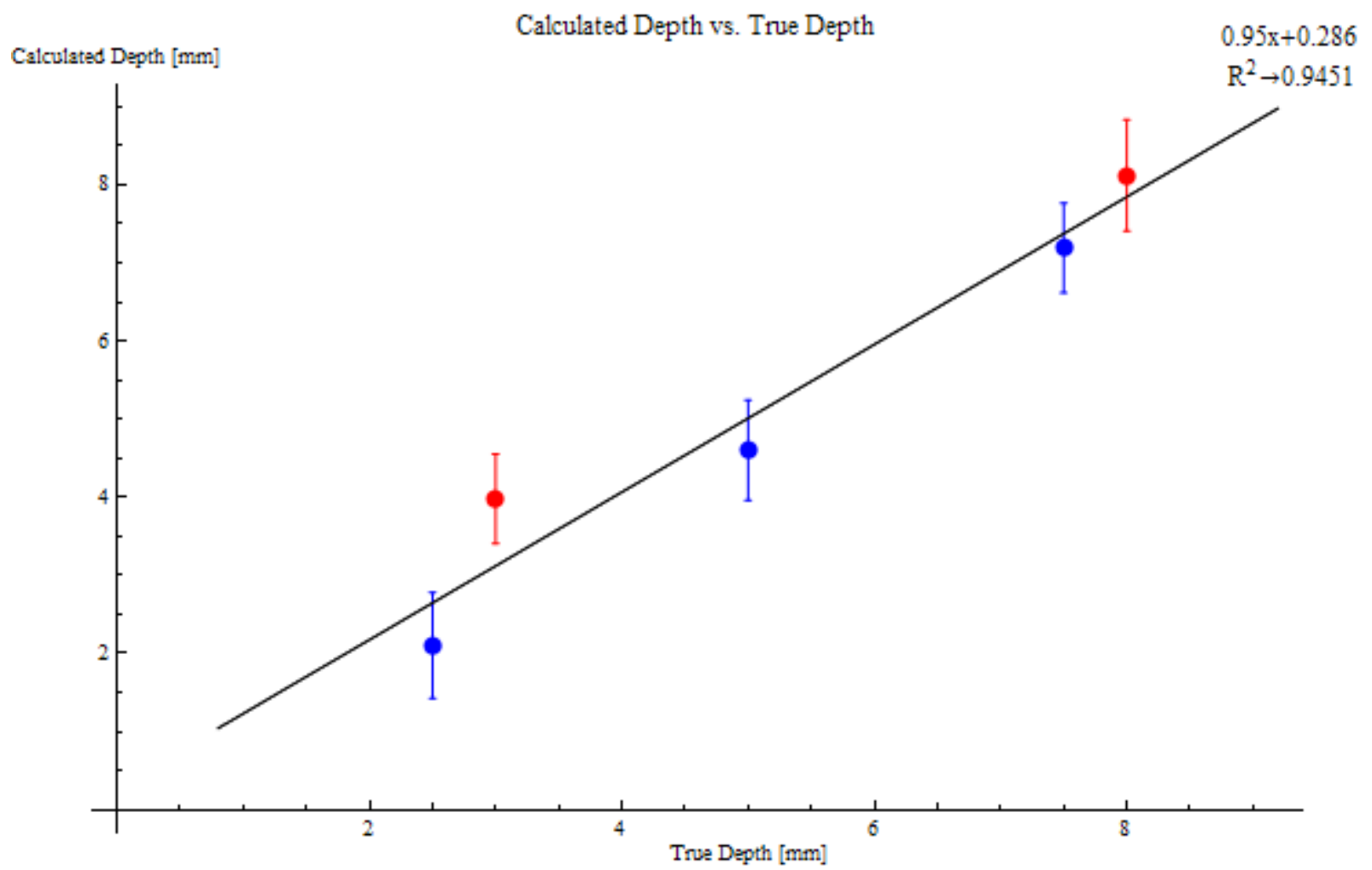

Fig. 6.6 Plot of calculated depth as a function of true depth. The error bars equal the FWHM of each DOI response peak in Fig. 6.4. The slope of the linear fit indicates the accuracy of the calibration function from Fig. 6.5.

While the dual ended readout approach for extracting DOI information presents a trade off between DOI sensitivity and energy resolution (as discussed in section 4.4.2.1) both the energy resolution and depth performance obtained for the single selected LYSO pixel exceeded expectation and provided a strong indication that such performance expectations could be extended to the bulk of the scintillation array pixels. A prototype prostate probe utilizing the SensL based DOI detector module is shown in Fig. 6.7 and demonstrates the capability of turning the laboratory based DOI detector module into a practical compact device for clinical use. However, robust case aside, the operational stability of the SensL SiPMs proved to be extremely unreliable throughout testing. This was particularly problematic as a unique pixel specific calibration function for each pixel in a scintillation array is necessary in order to maximize the DOI performance of the detector module. Therefore, further studies utilizing SensL SiPMs were not preformed. Nevertheless, the SensL based DOI detector module allowed for a successful proof of concept demonstration. 


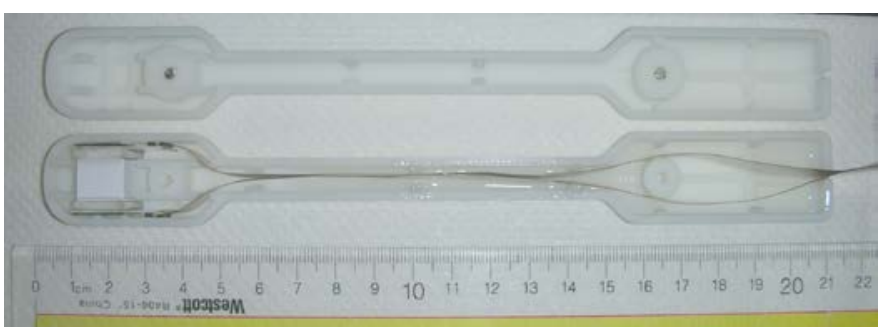

Fig. 6.7 Compact ( 14 $\mathrm{mm}$ wide by $\sim 20 \mathrm{~mm}$ high) prosate probe prototype based on the SensL dual ended readout detecotr modle. Case manufactrued by Agile Technologies.

\subsection{Hamamatsu Pilot and Full Scale Studies}

Pilot and full scale studies were preformed utilizing the apparatus and Hamamatsu SiPM (S109433344MF-050 MPPC) (Fig. 6.8a) based DOI detector module (Fig. 6.8c-d) discussed in section 4.3. The pilot study consisted of a systematic performance analysis of five scintillation pixels while the full scale study consisted of a systematic performance analysis of all 324 pixels comprising the scintillation array. During the pilot studies the second coincidence detector module consisted of a $1 \mathrm{~cm}$ diameter Hamamatsu R1635 PMT optically coupled to a $1 \mathrm{~cm}^{3}$ LYSO scintillator, while a Hamamatsu H8500 PMT optically coupled to a 2 x 2 array of $12 \times 12$ element LYSO scintillation arrays comprised of $1.0 \mathrm{~mm} \times 1.0 \mathrm{~mm} \times$ $10.0 \mathrm{~mm}$ pixels served as the coincidence detector during the full scale studies.

(a)

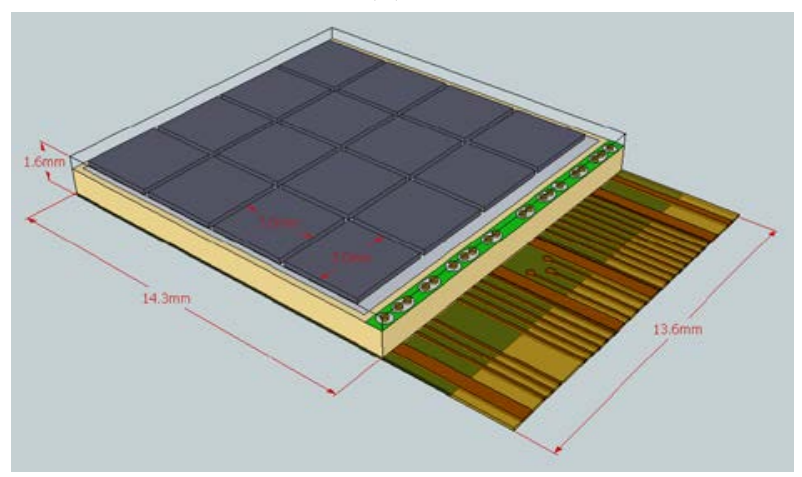

(b)

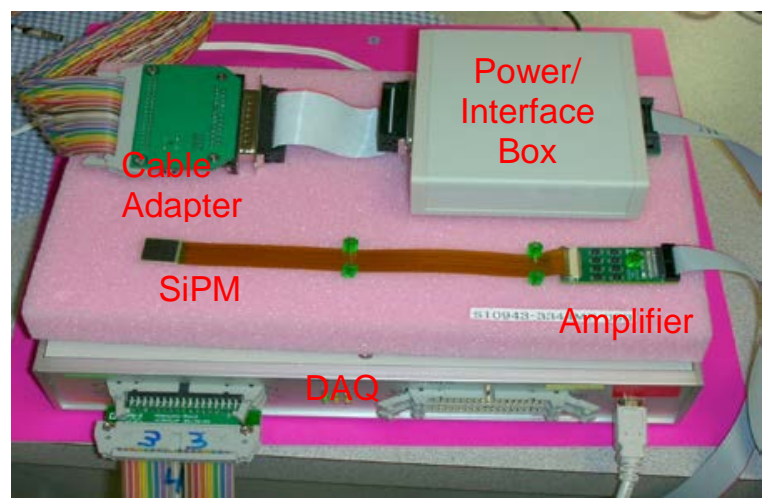

(c)

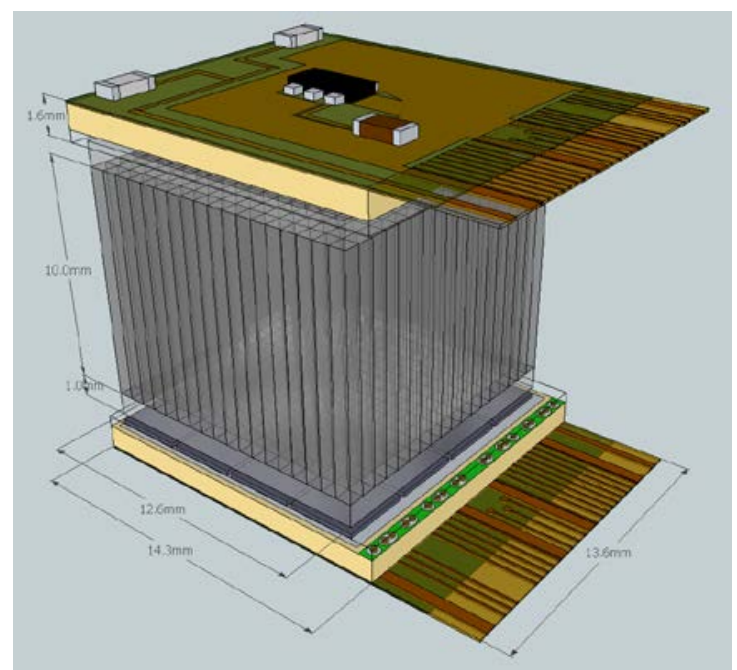

(d)

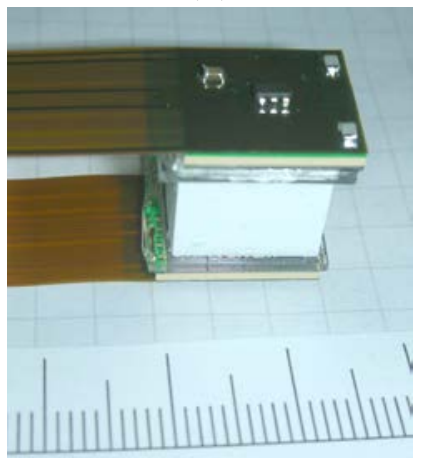

Fig. 6.8 (a) Hamamatsu SiPM schematic diagram, (b) various components of the experimental apparatus, (c) schematic diagram of the Hamamatsu SiPM based DOI detector module with approximate dimensions of $14.3 \mathrm{~mm}$ x $13.6 \mathrm{~mm}$ x $15.2 \mathrm{~mm}$, (d) assembly of the Hamamatsu SiPM based DOI detector module prior to laboratory test (mm scale). 


\subsubsection{Methods}

Prior to performing any DOI studies both energy and mutual gain calibrations, as outlined in section 6.2.1, were preformed on the DOI module. The flood histogram acquired from one of the SiPMs upon uniformly irradiating the DOI module during the energy calibration is shown in Fig. 6.9a. Aside from the outer edge of pixels all the LYSO pixels are visible and well differentiated. Following the energy calibration the mutual gain calibration was preformed. However, since each SiPM operates under a single applied reverse bias voltage i.e. a non-pixel specific bias, the five LYSO pixels on which subsequent DOI studies would be preformed were selected in a single ROI (region of interest) and the mutual gain calibration preformed.

(a)

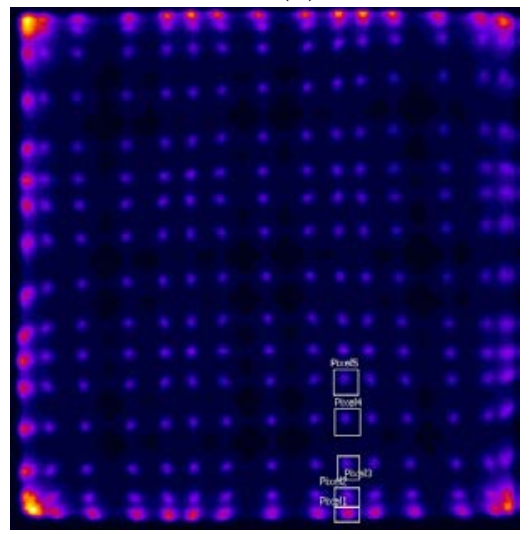

(b)

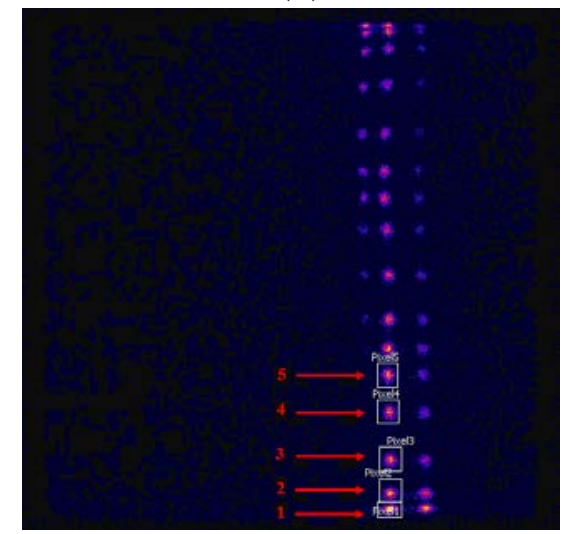

Fig. 6.9 (a) flood histogram obtained upon uniform irradiation of the DOI module, (b) flood histogram obtained for one SiPM upon irradiating the DOI module with a narrow electronically collimated ${ }^{22} \mathrm{Na}$ needle source. The five selected LYSO pixels are indicated by the red arrows.

Pilot DOI studies on the five selected LYSO pixels were performed using the geometry depicted in Fig. 6.10. Separation distance between the DOI module and the coincidence module was $10 \mathrm{~cm}$. A ${ }^{22} \mathrm{Na}$ needle source was mounted on a linear step motor with the radioactive tip positioned between both detector modules ( $1 \mathrm{~mm}$ from the DOI module). The needle source was used to probe the DOI module scintillation array in depth from $1 \mathrm{~mm}$ to $9 \mathrm{~mm}$ in $1 \mathrm{~mm}$ step. The flood histogram acquired for one SiPM upon irradiating the DOI module with the electronically collimated ${ }^{22} \mathrm{Na}$ needle source is shown in Fig. 6.9b. Unlike in the mutual gain calibration, separate ROIs were placed around each of the five pixels. The five selected LYSO pixels are numerically labeled from bottom to top in ascending order (Fig. 6.9b). Therefore, for each of the nine depths each of the five LYSO pixels was separately evaluated. Using equation 6.1 beam width incident on pixel 1 was estimated to be $0.607 \mathrm{~mm}$.

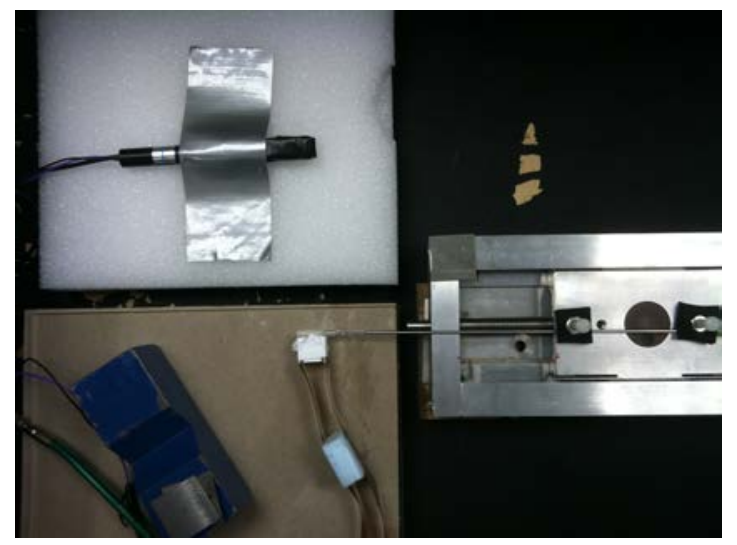

Fig. 6.10 Experimental geometry utilized during pilot DOI studies on five selected LYSO pixels. A ${ }^{22} \mathrm{Na}$ source was used to scan in depth from $1 \mathrm{~mm}$ to $9 \mathrm{~mm}$ in $1 \mathrm{~mm}$ steps. 
After performing a detector wide (over all LYSO pixels) mutual gain calibration full scale studies were preformed utilizing an altered geometry from that depicted in Fig. 6.10. A larger coincidence detector was utilized in order to create an electronically collimated $511 \mathrm{keV}$ fan beam broad enough in height to fully irradiate all the DOI module scintillation pixels at a particular depth. Separation distance between the DOI module and the coincidence module was $10 \mathrm{~cm}$, however instead of both modules remaining stationary the DOI module was mounted to the linear step motor. In between both modules was a mount used to hold a stationary capillary tube $(0.5 \mathrm{~mm}$ inner diameter $)$ filled with a solution of ${ }^{18} \mathrm{~F}$ in place $1 \mathrm{~mm}$ from the DOI module. The DOI module was progressively stepped and irradiated at eight $1 \mathrm{~mm}$ increments. In an effort to further narrow the fan beam width yet keep the coincidence module close enough to the capillary tube in order to gain adequate statistics a mask was placed between the scintillation arrays and the PMT covering all scintillation pixels except the single column of pixels of which was alighted with the capillary tube. Using equation 6.1 beam width incident on the side of the DOI module closest to the capillary tube was estimated to be $0.515 \mathrm{~mm}$.

\subsubsection{Pilot Study Results}

\section{Energy Characterization}

A Gaussian + quadratic background fit was applied to the sum energy spectra of each LYSO pixel at each particular irradiation depth. The energy resolution as a function of true depth is shown for each pixel in Fig. 6.12. The energy resolution averaged over all depths for each LYSO pixel is shown in Table 6.1. When averaged over all depths pixel 1 exhibited the worst energy resolution of $14.8 \pm 2.1 \%$ while pixels 2 through 5 exhibited the best energy resolution with a cumulative average of $11.4 \pm 1.5 \%$. When averaged over all pixels and all depths energy resolution was $12.0 \pm 2.1 \%$.

The histogram of the signal from SiPM-A verses the signal from SiPM-B for pixel 2 is depicted in Fig. 6.11. The bright crest corresponds to $511 \mathrm{keV}$ photopeak events with each cluster corresponding to one of the nine incremental irradiation depths.

The centroid location of the $511 \mathrm{keV}$ photopeak as a function of true depth (the depth at which the DOI module was irradiated) for both SiPM-A (purple squares), SiPM-B (gold diamonds), and the sum energy spectra (blue circles) are shown in Fig. 6.13. The number of scintillation photons, and thus the energy incident on each SiPM, is depth dependent. As discussed prior, the mechanisms for photon reduction include the rough lateral surfaces of each LYSO pixel, as well as the diffuse white reflector used to optically isolate each pixel within the scintillation array. Therefore, the position of the $511 \mathrm{keV}$ photopeak centroid for both SiPM-A and SiPM-B is governed by equation 1.21. However, as discussed in section 4.4.2.1 the attenuation coefficient $\mu$ is replaced by an effective attenuation coefficient $\mu_{\mathrm{e}}$. Using equation 1.2.1 as a model, exponential fits were applied to both SiPM-A and SiPM$B$ data sets. A quadratic fit was applied to the sum data set for each individual LYSO pixel. When averaged over all five pixels $\mu_{\mathrm{e}}$ as measured by SiPM-A was $0.141[1 / \mathrm{mm}]$ and $0.155[1 / \mathrm{mm}]$ for SiPM-B.

Table 6.1

\begin{tabular}{|c|c|}
\hline Pixel & Average Energy Resolution (\%) \\
\hline 1 & $14.8 \pm 2.1$ \\
\hline 2 & $11.5 \pm 1.9$ \\
\hline 3 & $11.2 \pm 1.6$ \\
\hline 4 & $11.4 \pm 1.4$ \\
\hline 5 & $11.4 \pm 1.3$ \\
\hline $2-5$ & $11.4 \pm 1.5$ \\
\hline A11 & $12.0 \pm 2.1$ \\
\hline
\end{tabular}

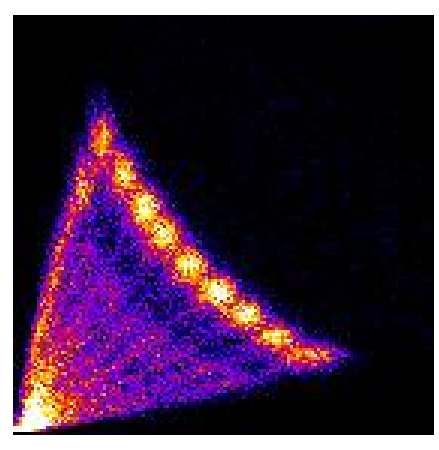

Fig. 6.11 histogram of the signal from SiPM-A vs. SiPM-B. The bright crest corresponds to 511 $\mathrm{keV}$ photopeak events with each cluster corresponding to one of the nine incremental irradiation depths 


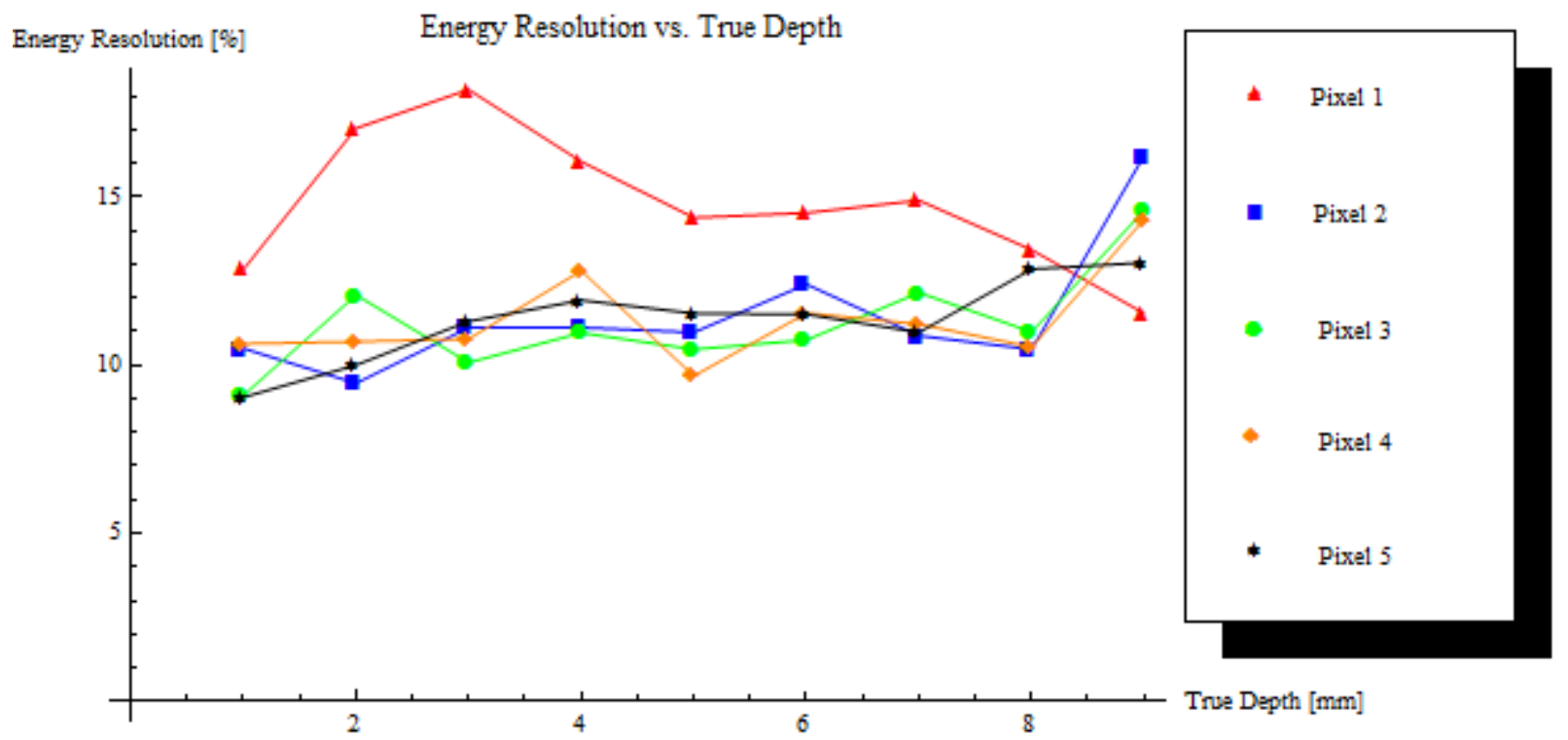

Fig. 6.12 Energy resolution as a function of true depth for each of the five selected LYSO pixels.

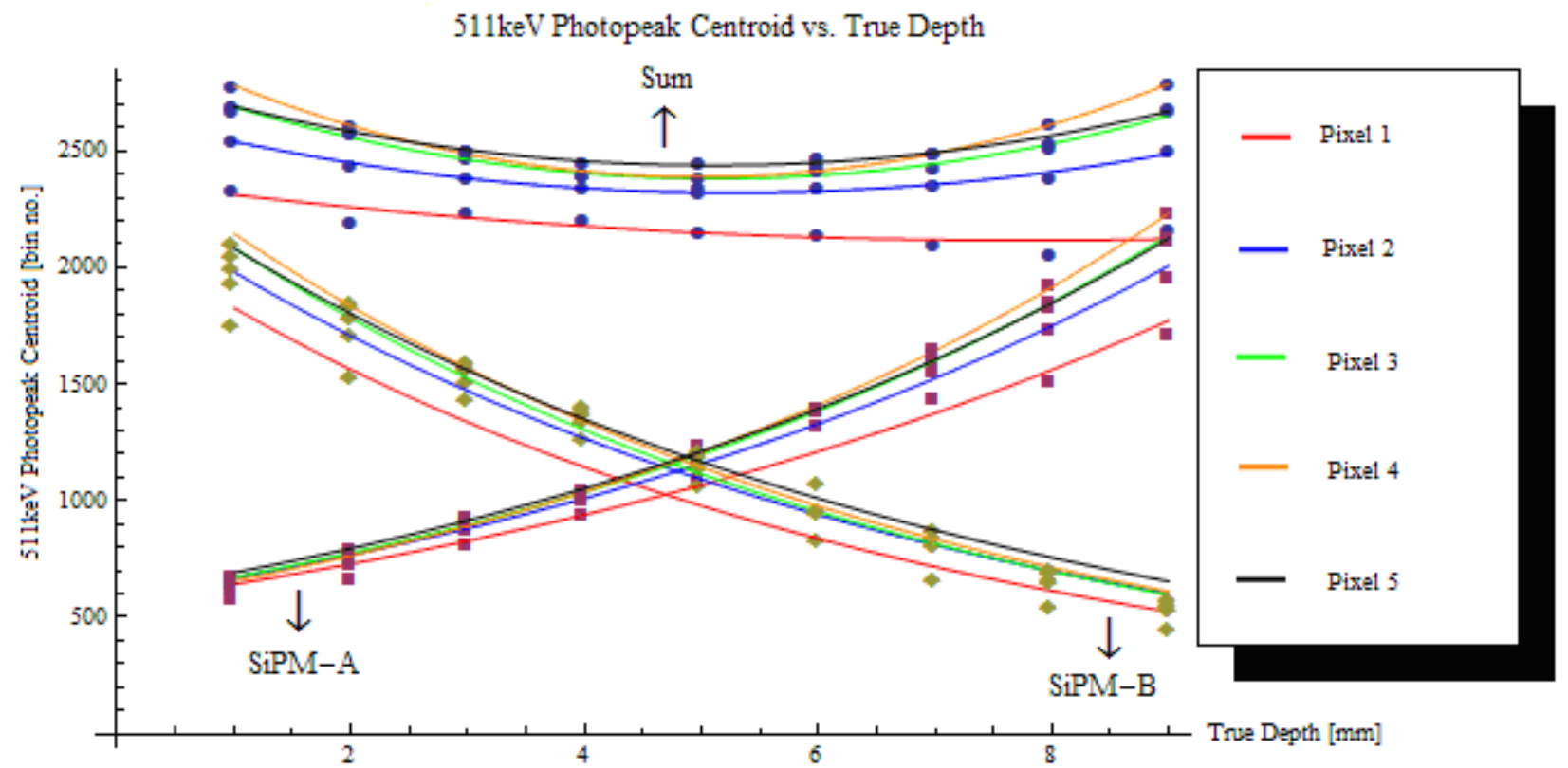

Fig. 6.13 $511 \mathrm{keV}$ photopeak centroid location as a function of depth for the energy spectra of SiPM-A, SiPM-B, and the sum energy spectra.

\section{$\underline{\text { Extraction of DOI Information }}$}

The DOI profiles for the five selected LYSO pixels are shown in Fig. 6.14. Each peak was generated by applying a Gaussian + quadratic background fit to the binned DOI ratio data readout from Kmax. As was discussed in section 6.2.2 a pixel specific linear calibration function based on known ratios of distance in millimeters to distance in histogram bins was determined for each pixel. The linear calibration functions and corresponding $\mathrm{R}^{2}$ values are shown in Fig. 6.15. Averaging over all depths pixel 1 demonstrated the best DOI resolution of $0.716 \pm 0.086 \mathrm{~mm}$ while pixel 5 demonstrated the worst DOI resolution of $0.836 \pm 0.072 \mathrm{~mm}$. When averaged over all depths and all five pixels DOI resolution was found to be $0.750 \pm 0.086 \mathrm{~mm}$. 

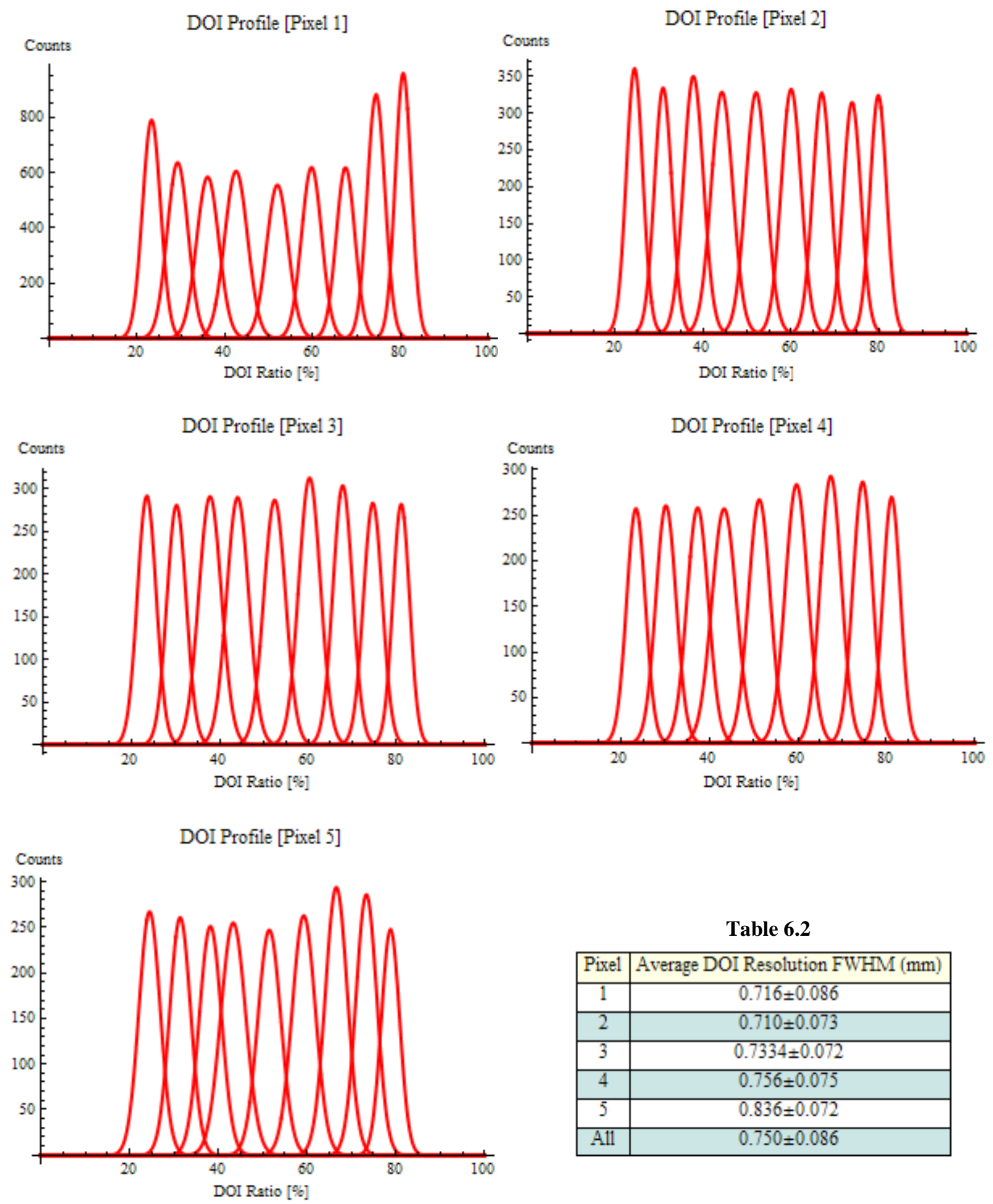

Table 6.2

\begin{tabular}{|c|c|}
\hline Pixel & Average DOI Resolution FWHM (mm) \\
\hline 1 & $0.716 \pm 0.086$ \\
\hline 2 & $0.710 \pm 0.073$ \\
\hline 3 & $0.7334 \pm 0.072$ \\
\hline 4 & $0.756 \pm 0.075$ \\
\hline 5 & $0.836 \pm 0.072$ \\
\hline All & $0.750 \pm 0.086$ \\
\hline
\end{tabular}

Fig. 6.14 DOI profiles for pixels 1-5. Each peak corresponds to a $1 \mathrm{~mm}$ incremental irradiation depth from $1 \mathrm{~mm}$ to $9 \mathrm{~mm}$. 


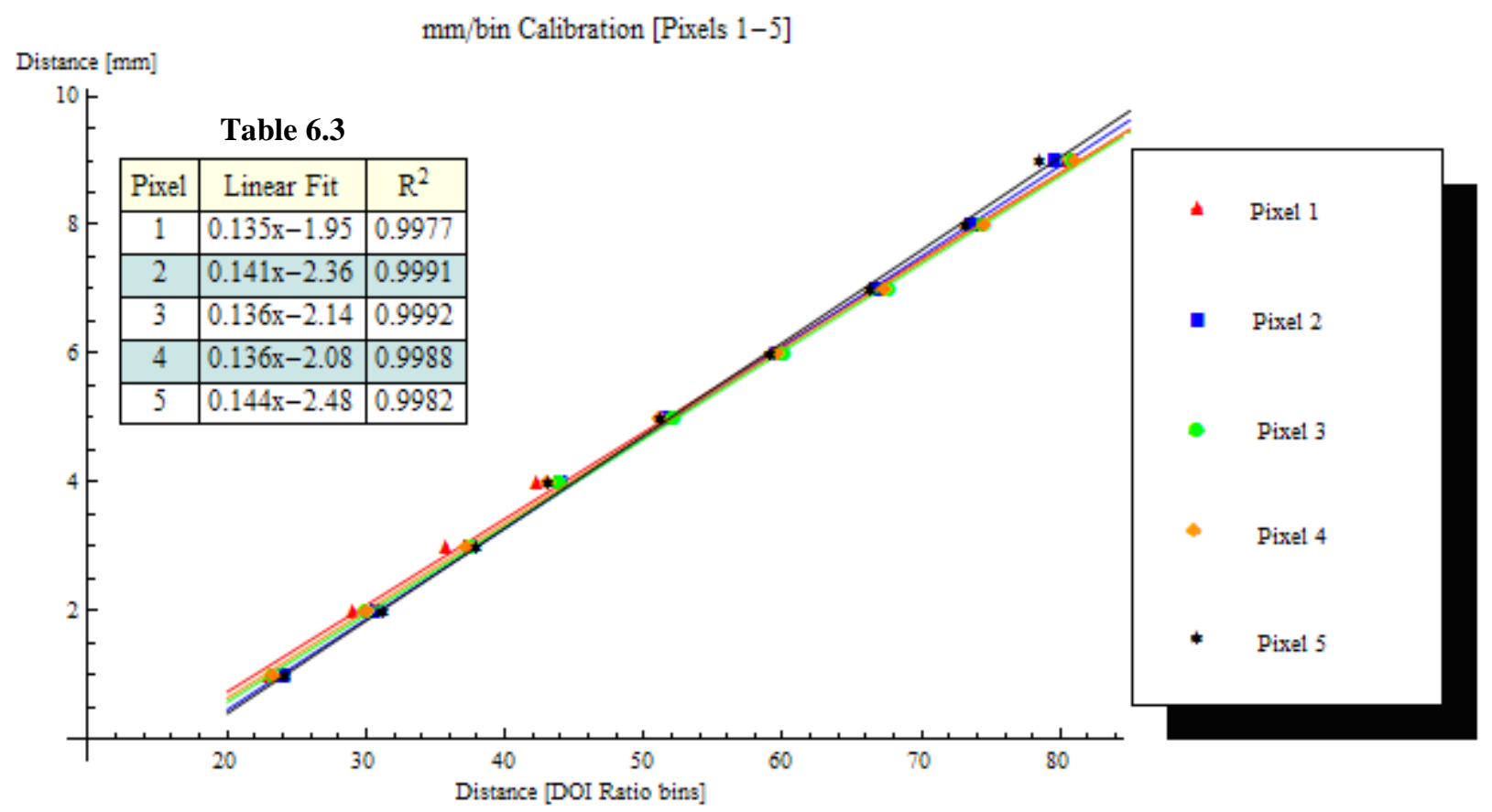

Fig. 6.15 Plot of the known mm/bin ratios from for each of the DOI profiles in Fig. 6.14. A linear fit was applied to each data set with the slope of each fit equal to the pixel specific $\mathrm{mm} / \mathrm{bin}$ calibration factor $\mathrm{k}$.

\subsubsection{Full Scale Results}

During full scale studies, data was readout in a continuous list mode file for each irradiation depth. For each coincidence event detected, 22 bytes of data was readout. Each 22 byte set was comprised as follows: SiPM-A signal (4 bytes), SiPM-B signal (4 bytes), sum geometric mean energy (4 bytes), DOI ratio ( 4 bytes), the $x$-coordinate of interaction ( 2 bytes), the y-coordinate of interaction ( 2 bytes), and the sum energy ( 2 bytes). The data contained in each list mode file was partitioned and sorted using IDL (Exelis Visual Information Solutions) such that events corresponding to the same pixel were used to generate pixel specific energy spectra, DOI ratio histograms, and calibration functions for all 324 LYSO pixels.

\section{Energy Characterization}

Utilizing the sum energy spectra from each of the 324 LYSO pixels at each of the eight irradiation depths the overall DOI module energy spectra averaged over all pixels and all depths is shown in Fig. 6.16. The overall average energy spectra was calculated as the geometric mean in order to correct for exponential light decay. A Gaussian + quadratic background fit was applied to the overall average energy spectra yielding a mean energy resolution of $13.2 \pm 0.7 \%$ across the entire DOI module.

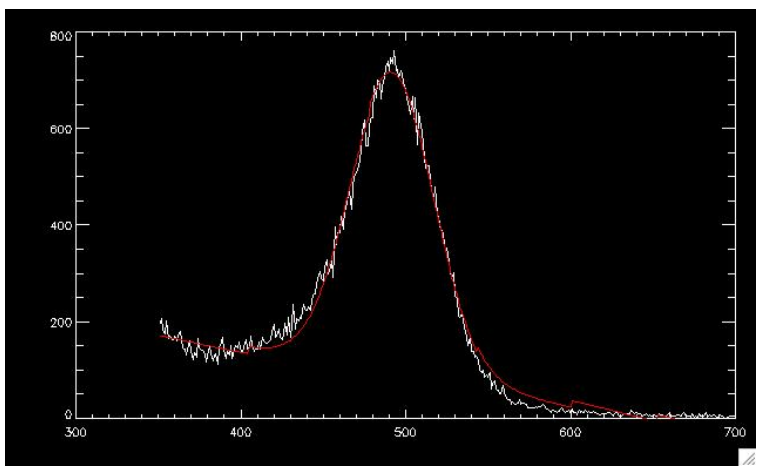

Fig. 6.16 Overall DOI module energy spectra calculated as the geometric mean and averaged over all 324 LYSO pixels and all nine irradiation depths. 


\section{Extraction of DOI Information}

The overall DOI profile is shown in Fig. 6.17. Each peak corresponds to the mean DOI response when averaging over all 324 LYSO pixels as each of the eight irradiation depths. Utilizing the overall DOI profile an average detector wide calibration function was determined and used to convert the x-axis of Fig. 6.17 from bins to millimeters. The FWHM of each of the eight Gaussian peaks was averaged yielding a mean DOI resolution of $0.78 \pm 0.09 \mathrm{~mm}$ across the entire DOI module.

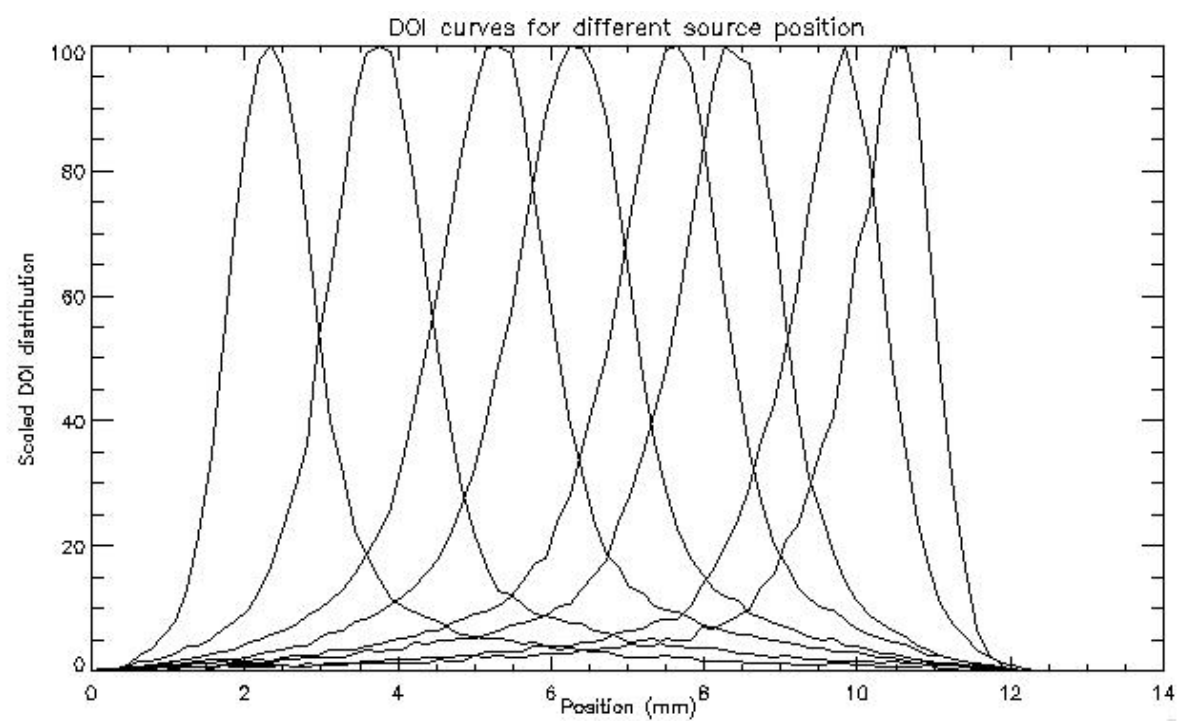

Fig. 6.17 Overall DOI profile with each peak corresponding to the mean DOI response when averaging over all 324 LYSO pixels at each particular irradiation depth.

\subsubsection{Discussion}

Energy resolution averaged over all depths and all five LYSO pixels was excellent. Aside from the $9 \mathrm{~mm}$ irradiation depth, pixels 2-5 demonstrated a nearly depth invariant energy response. Pixel 1 , however demonstrated a, worse as well as, varying energy response with depth. The decrease in energy resolution of pixels 2-5 at the $9 \mathrm{~mm}$ irradiation depth, as well as the overall worse energy resolution of pixel 1 over all depths, can be attributed to the degraded sampling of scintillation photon at the edge of the detector. Edge effects such as light loss, crosstalk, and the non-uniform distribution of scintillation photons over the face of the SiPM are the prime mechanisms associated with worse sampling. More specifically, in contrast to interior pixels, of which produce a uniform cone of scintillation photons across the face of the $\mathrm{SiPM}$, the light cone of edge pixels is truncated and compressed. The non-uniformity of the light cone not only leads to degraded energy resolution, but also results in inaccurate center of gravity calculations causing pixel bunching, overlap, and general decrease in spatial resolution at the edge of the detector.

The depth invariant energy response for pixels 2-5 suggests a linear relationship between light yield and depth and thus a linear relationship between the signal from SiPM-A and SiPM-B. However, the histogram of the signal from SiPM-A vs. the signal from SiPM-B (Fig. 6.11) as well as the exponential nature of the $511 \mathrm{keV}$ centroid location illustrated in Fig. 6.13 would appear to suggest otherwise. Although, upon further analysis the calculated values for the effective attenuation coefficient $\mu_{\mathrm{e}}$ are small enough in magnitude that equation 1.21 can be approximated as linear over short distances. Therefore, not only can light yield be approximated as depth independent for interior scintillation pixels but a linear relationship between DOI ratio and depth can be approximated as well.

It should also be noted that the nine clearly differentiable clusters in Fig. 6.11 indicate an average 
minimum DOI resolution of $1 \mathrm{~mm}$ for pixel 2. As a result of the nearly identical energy response of pixels 2-5 this minimum DOI resolution expectation can also be extended to pixels 3-5.

As was discussed in section 6.2.3 the calibration functions calculated for each pixel (Fig. 6.15) are only as useful as they are accurate. The accuracy of each calibration function was determined pixel by pixel by plugging the centroid values of each peak from the corresponding pixel DOI profile into the calibration equation and plotting the resultant calculated depth as a function of true depth (Fig. 6.18). The error bars in Fig. 6.18 correspond to the FWHM of each of the DOI response peaks in Fig. 6.14. A linear fit was applied to each data set with the slope and corresponding $\mathrm{R}^{2}$ value of each fit indicated in Table 6.4. The statistically identical fits for each data set illustrate a uniform DOI response across each of the five pixels. Also, with both the slope and $\mathrm{R}^{2}$ value of each fit approaching one the uncertainty in the calibration function of each pixel approaches zero.

Calculated Depth vs. True Depth [Pixels 1-5]

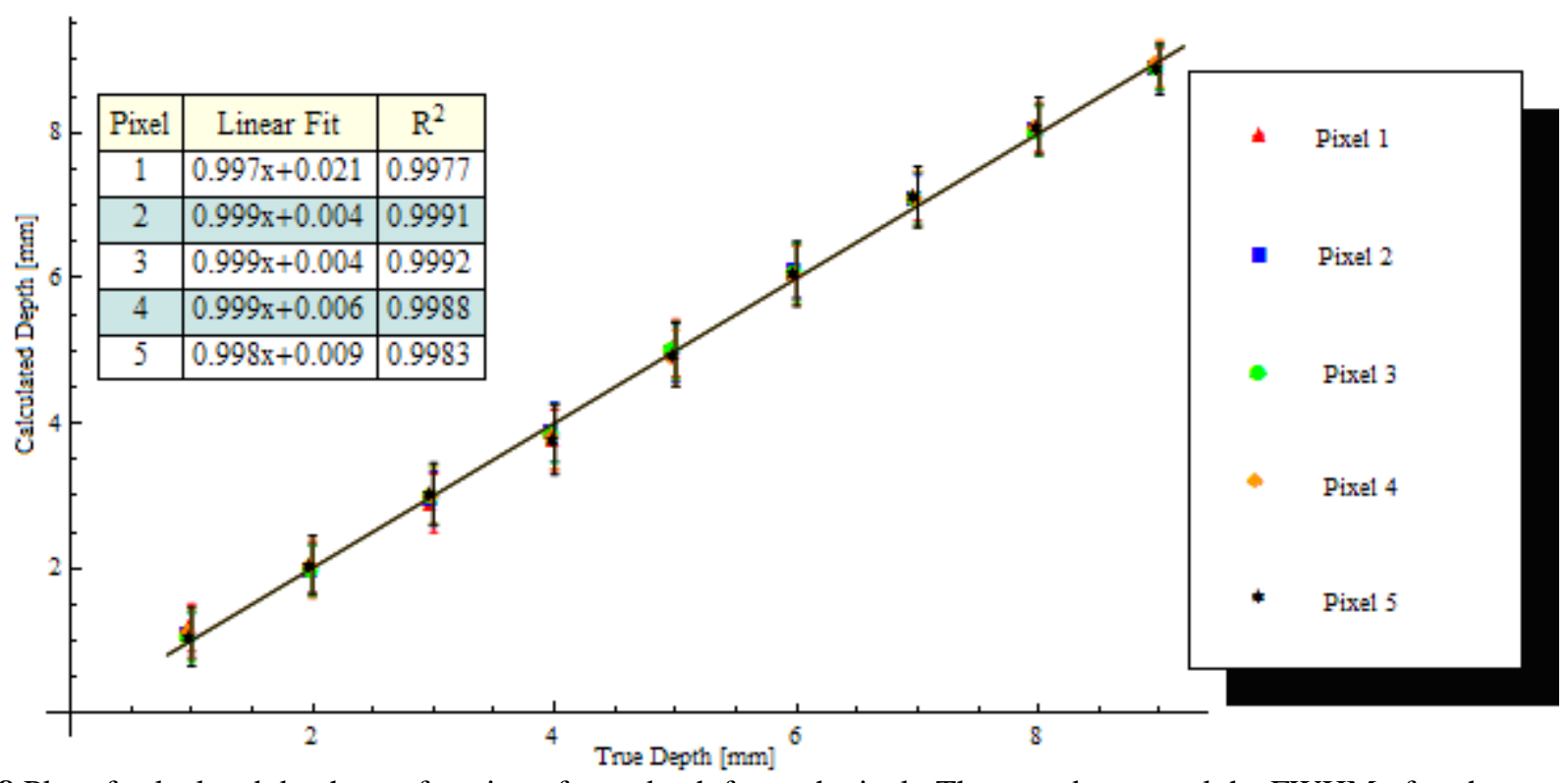

Fig. 6.18 Plot of calculated depth as a function of true depth for each pixel. The error bars equal the FWHM of each DOI response peak in Fig. 6.14. The slope of each linear fit indicates the accuracy of the calibration function for each pixel.

It should be noted that, while the DOI response across all five pixels was uniform and sub-mm DOI resolution for each pixel was obtained, resolution progressively decreased along the plane perpendicular to the axial length of the scintillation array. This decrease in DOI resolution is attributed to beam broadening (i.e. an increasing value of $b$ in equation 6.1 as the beam progresses from pixel 1 to pixel 5) and manifests itself in the increasingly board DOI profile distributions from pixel 1 to pixel 5. While the effect of beam broadening on DOI resolution was not corrected for in the analysis of pixels 1-5 it was however taken into account and corrected for when calculating the overall mean DOI resolution across the entire DOI module.

\subsection{Image Reconstruction and DOI Information}

While the performance characterization of the above Hamamatsu based DOI module continues, concurrent studies utilizing a separate dual ended readout detector module were preformed in order to determine if there is any significant performance gain in reconstruction image quality when incorporating 
DOI information extracted from a small compact DOI based PET detector module.

The dual ended readout module was comprised of two 5 x 5 element Hamamatsu SiPMs (S10943-9059(X)) optically coupled to the opposing axial ends of a 24 x 24 element scintillation array comprised of saw cut $1 \times 1 \times 10 \mathrm{~mm}^{3}$ LYSO pixels. Each pixel was optically isolated in the array via $50 \mu \mathrm{m}$ thick Toray Lumirror E60 septa. A $2.8 \mathrm{~mm}$ acrylic light spreader window was optically coupled between both SiPM and scintillation array interfaces (Fig. 6.19). Unlike the above dual ended readout modules, a resistive readout scheme was utilized thus reducing the number of readout channels from 25 to 4 (Majewski, 3939-3944). The second coincidence module was a $20 \times 15 \mathrm{~cm}$ panel detector comprised of 4 x 3 array of Hamamatsu H8500 PMTs coupled to LYSO scintillation arrays with a pixel pitch of $2 \mathrm{~mm}$.
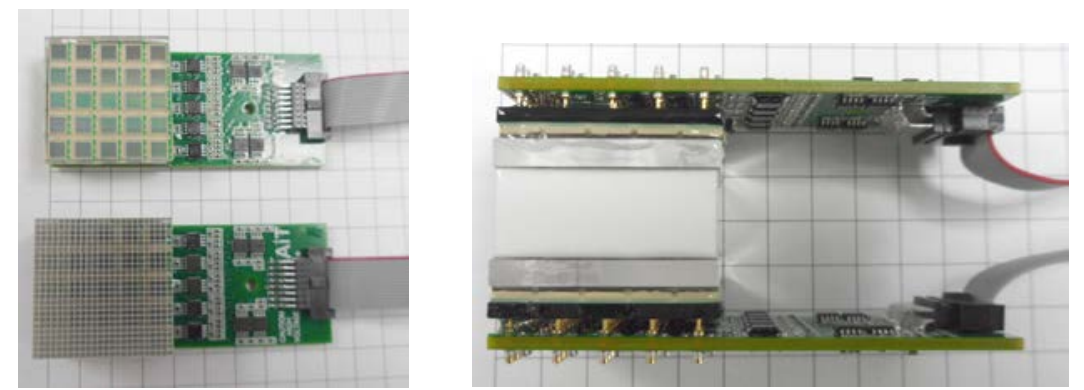

Fig. 6.19 Dual ended resistive readout module based on a 5 x 5 array of Hamamatsu SiPMs. The four channel resistive readout was manufactured by AiT Instruments. Both photos are against a $6 \mathrm{~mm}$ scale background.

\section{$\underline{\text { 6.4.1 Methods }}$}

Prior to utilizing the dual ended resistive readout detector module in image reconstruction studies a detector wide DOI calibration function was determined and applied to each pixel in the scintillation array. While not optimal (i.e. a proprietary calibration function for each LYSO pixel in the scintillation array) a rough DOI calibration was sufficient for the subsequent studies.

In an effort to amplify the effects of incorporating DOI information into the reconstruction algorithm, studies were preformed in a close geometry with the DOI module angled $40^{\circ}$ relative to the panel detector thus increasing the fraction of obliquely incident $511 \mathrm{keV}$ gammas. Separation distance between the DOI module and the coincidence panel detector was approximately $8 \mathrm{~cm}$. An arrow phantom consisting of 25 two millimeter diameter sieves beads soaked in ${ }^{18} \mathrm{~F}$ was positioned $5 \mathrm{~cm}$ from the panel detector. Separation distance between the beads comprising the head of the array was $5 \mathrm{~mm}$, while the beads separating the diagonal tail were separated by $7.1 \mathrm{~mm}$ (Fig. 6.20).

(a)

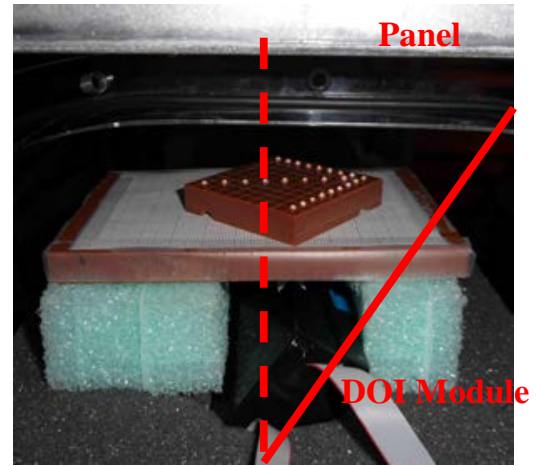

(b)

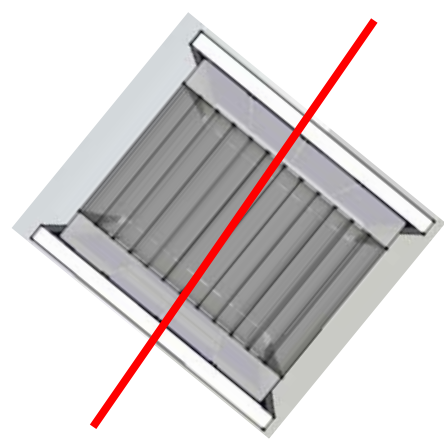

Fig. 6.20 (a) Resistive DOI module angled $40^{\circ}$ relative to the panel detector. Separation distance as approximately $8 \mathrm{~cm}$ with an arrow phantom positioned $5 \mathrm{~cm}$ from the panel detector. (b) DOI module orientation relative to the panel detector. 


\section{$\underline{6.4 .2}$ Results}

The effects of incorporating DOI information into a laminography type reconstruction algorithm are illustrated in Fig. 6.21. Both the reconstructed image (column (a)) and the associated projection of each image (column (b)) are shown when DOI information is not incorporated into the reconstruction algorithm (top row) and when DOI information is incorporated (bottom row). While both reconstructed images resolve all nine beads spaced $7.1 \mathrm{~mm}$ apart and 13 beads spaced $5 \mathrm{~mm}$ apart the beads are appreciably sharper and better differentiated throughout the FOV when DOI information is incorporated. This assertion is quantitatively supported by the difference in peak-to-valley ratios of the image projections shown in (b1) and (b2). When no DOI correction was incorporated into the reconstruction algorithm the average peak-to-valley ratio of the image projection was 2.525 (b1) as compared to 4.757 when DOI correction was incorporated (b2). From a qualitative standpoint, the peaks in (b2) are much better defined and resolvable as compared to the peaks in (b1).

(a1)

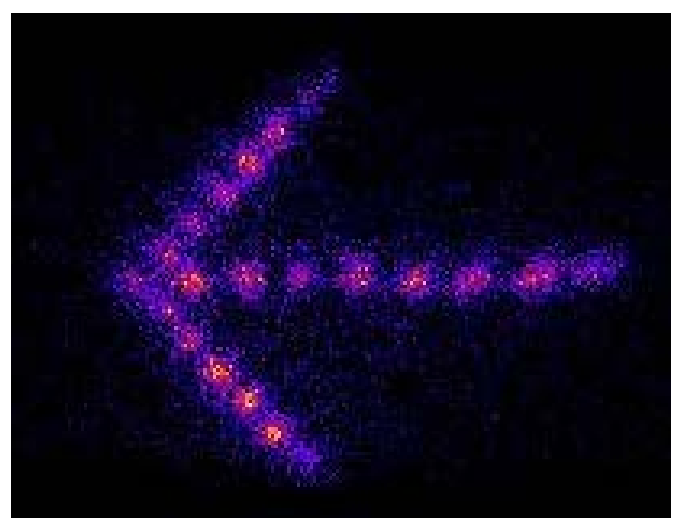

\section{$\underline{\text { No DOI Correction }}$}

DOI Correction

(a2)

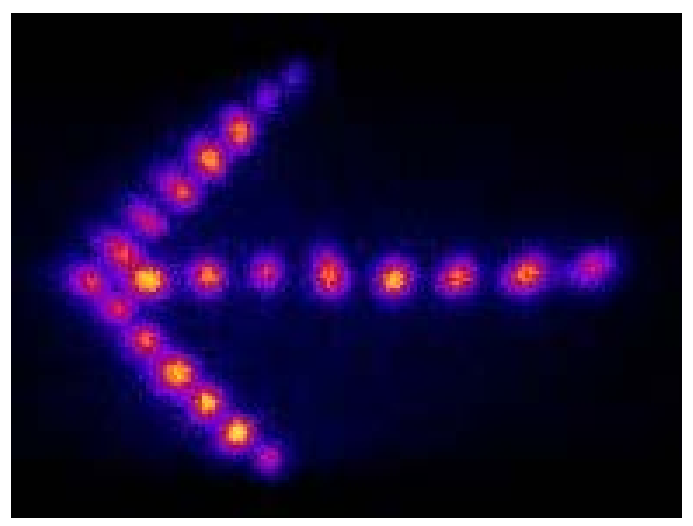

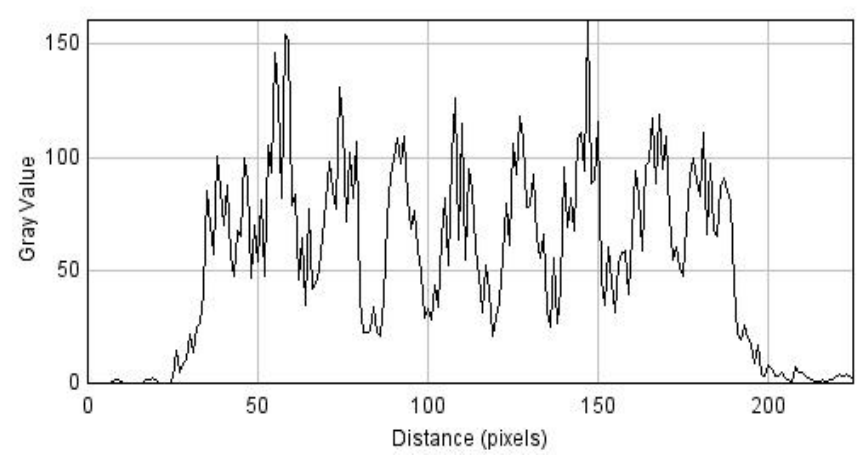

(b1)

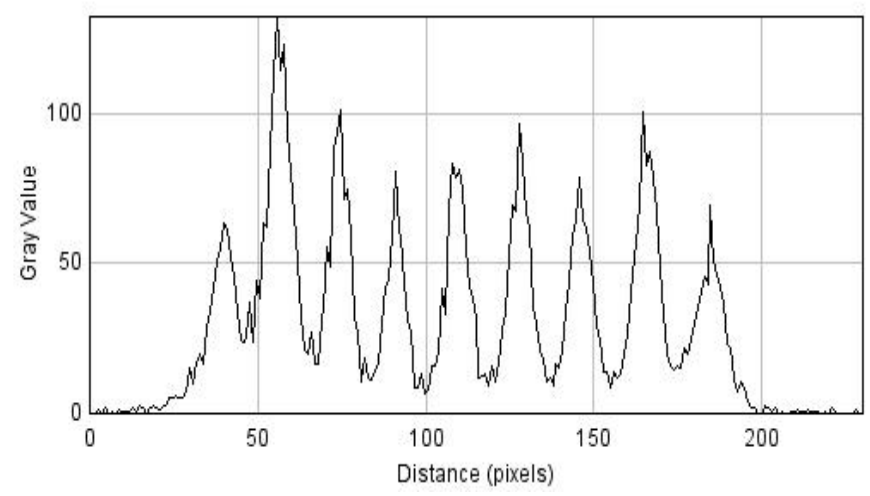

Fig. 6.21 Effects of DOI correction on reconstruction image quality.

\subsubsection{Discussion}

Depth of interaction information is incorporated into the reconstruction algorithm such that the $\mathrm{x}-\mathrm{y}$ coordinate of energy deposition within a scintillation crystal is assigned an event specific 
depth instead of being projected to a default depth (typically either the inner face of the center of the scintillation crystal). Consequently, LORs more accurately correspond to the path along which back to back annihilation events occurred.

In the dual ended resistive readout module + top panel geometry incorporation of DOI information into the reconstruction algorithm not only improved image quality but also improved the uniformity of spatial resolution throughout the FOV. Demonstration of such a significant improvement in image quality and uniformity of spatial resolution via a compact DOI based detector module complements simulations and experimental efforts by others (Kao, 1-11; St. James, 4605-4619) while simultaneously validating the approach of utilizing such a detector module as a cost effective, mobile, and high efficiency supplemental probe to increase the overall qualitative accuracy of reconstructed images.

\subsection{Overview}

As a result of new prostate specific radiopharmaceuticals as well as a trend towards organ and purpose specific PET imagers the development and performance evaluation of a prostate specific PET detector module has been carried out at West Virginia University. The detector module is both small and compact and of a dual ended readout design thus providing vital DOI information used to minimize the effects of parallax error in reconstructed images. While the SensL based DOI module proved problematic both Hamamatsu based modules exceeded expectations. The observed significant increases in image quality upon incorporation of DOI information from a small compact DOI module along with the archaic methods currently used to screening for and diagnosis prostate cancer provide a healthy motivation to continue further studies. Such studies include focusing on the smaller non-resistive Hamamatsu based module and transforming the laboratory based module into both a clinically and anatomically practical probe for endorectal insertion. Even further studies include evaluating the probe's performance when subjected to prostate phantom studies while concurrently incorporating each pixel specific calibration function into the reconstruction algorithm. While the main focus is to aid in prostate cancer diagnosis and biopsy guidance, such a probe could also aid in gynecological imaging of cervical cancer, imaging vulnerable plaque in carotid arteries, and or probing for cancerous breast lesions.

\section{References}

Adaptive I/O Technologies, Inc. http://www.ait-instruments.com/.

Agile Technologies. http://www.agiletechnologies.net/.

American Cancer Society, Prostate Cancer, [Online] Available: http://www.cancer.org/Cancer/ProstateCancer/DetailedGuide/index , Atlanta, GA, 2012.

Exelis Visual Information Solutions. http://www.exelisvis.com/ProductsServices/IDL.aspx.

Hamamatsu Photonics K. K. http://sales.hamamatsu.com/.

Hara, T., N. Kosaka, and H. Kishi, “PET Imaging of Prostate Cancer Using Carbon-11-Choline.” Journal of Nuclear Medicine, vol. 39, issue 6, pp. 990-995, 1998.

Kao, C-M., Dong, Y. Xie, Q., and Chen, C-T., “Accurate Image Reconstruction with Computed System Response Matrix for a High-Sensitivity Dual-Head PET Scanner.” submitted to: IEEE Transaction on Medical Imaging, pp. 1-11, May 2008.

Majewski, S., Proffitt, J., Stolin, A., and Raylman, R., “Development of a "Resistive” Readout for SiPM Arrays.” 2011 IEEE Nuclear Science Symposium Conference Record, pp. 3939-3944, 2011. 
Oehr, O., and Bouchelouche, K., "Imaging of Prostate Cancer.” Current Opinion in Oncology, vol. 19, issue 3, pp. 259-264, 2007.

Rohren, E. M., T. G. Turkington, and R. E. Coleman, “Clinical Applications of PET in Oncology.” Radiology, vol. 231, issue 2, pp. 305-332, 2004.

SensL. http://sensl.com/.

St. James, S., Y. Yang, Y. Wu, R. Farrell, P. Dokhale, K. S. Shah, and S. R. Cherry, "Experimental characterization and system simulations of depth of interaction PET detectors using $0.5 \mathrm{~mm}$ and $0.7 \mathrm{~mm} \mathrm{LSO}$ arrays." Physics in Medicine and Biology, vol. 54, issue 14, pp. 4605-4619, 2009. 


\section{ACKNOWLEDGEMENTS}

I would sincerely like to thank my research advisor Dr. Stanislaw Majewski under whose guidance this study was undertaken. His patience, kindness, encouragement, and insight have been paramount. I would also like to thank both my committee chair Dr. Raymond Raylman, and committee member Dr. Alan Bristow for their support and suggestions in the preparation of this thesis. I would like to express a deep gratitude to Dr. Alexander Stolin for his many insightful conversations and unabated willingness to drop what he was doing to help myself, as well as others. I would also like to thank Pete Martone for his support, insight, and friendship both inside and outside the lab. I would like to thank the faculty, staff, and fellow graduate students of both the Center for Advanced Imaging, as well as the Department of Physics, for their valuable and unwavering support along the way. Finally, I owe a deep debt of gratitude to both my family and friends who have stuck by me during this journey. 